\title{
Self-energy-part resummed quark and gluon propagators in a spin-polarized quark matter and generalized Boltzmann equations
}

\author{
A. Niégawa* \\ Graduate School of Science, Osaka City University, Sumiyoshi-ku, Osaka 558-8585, JAPAN \\ (Received today)
}

\begin{abstract}
We construct perturbative frameworks for studying nonequilibrium spinpolarized quark matter. We employ the closed-time-path formalism and use the gradient approximation in the derivative expansion. After constructing self-energy-part resummed quark and gluon propagators, we formulate two kind of mutually equivalent perturbative frameworks: The first one is formulated on the basis of the initial-particle distribution function, and the second one is formulated on the basis of "physical"-particle distribution function. In the course of construction of the second framework, the generalized Boltzmann equations and their relatives directly come out, which describe the evolution of the system. The frameworks are relevant to the study of a magnetic character of quark matters, e.g., possible quark stars.

11.10.Wx, 12.38.Mh, 12.38.Bx, 13.40.-f
\end{abstract}

Typeset using REVTEX

*Electronic address: niegawa@sci.osaka-cu.ac.jp 


\section{INTRODUCTION}

Recent possible discovery of a quark star $[1,2]$ renews our interest in the study of quark matters. It has been pointed out [3] the possibly of existing quark liquid in a ferro-magnetic phase. For analyzing the magnetic property of quark matters in a consistent manner [4-6], it is necessary to construct a self-energy-part resummed quark and gluon propagators in a spin-polarized quark matter, and thereby frame a perturbation theory.

The spin-polarized quark matter is, in general, out of equilibrium. For dealing with such systems, we employ the closed-time-path formalism [4,5]. In this formalism, propagators, vertices, and self-energy parts enjoy $(2 \times 2)$-matrix forms, denoted "^" . Let $\hat{G}(x, y)$ be a generic two-point function. Fourier transforming with respect to $x-y$ (Wigner transformation), we have $\hat{G}(P, X)$ with $X=(x+y) / 2$. We assume that $\hat{G}(P, X)$ depends weakly on $X$. Then, as usual, employing a derivative expansion (DEX), we use the gradient approximation:

$$
\hat{G}(P ; X) \simeq \hat{G}(P ; Y)+(X-Y)^{\mu} \partial_{Y^{\mu}} \hat{G}(P ; Y)
$$

We refer to the first term on the right-had side (RHS) as the leading part (term) and to the second term as the gradient part (term). Throughout this paper, we assume that the density matrix is color singlet, so that the quark and gluon propagators are diagonal in color space and independent of color index. Then, we drop the color index throughout.

The plan of the paper is as follows. In Sec. II, the leading term in the DEX of the selfenergy-part resummed (SEPR) quark propagator is constructed. In Sec. III, we construct the leading term of the SEPR gluon propagator in a Coulomb gauge. In Secs. II and III, the argument $X$ is dropped throughout. In Sec. IV, we present the gradient terms of the quark and gluon propagators. Then, we frame two mutually equivalent perturbative frameworks. One framework is constructed in terms of the "bare"-number-density function (and its relative), and the other, which we call physical- $N$ scheme, is constructed in terms of the "renormalized"-number-density function (and its relative). The latter scheme accompanies

the generalized Boltzmann equation for the "renormalized"-number-density function and its 
relatives. The form for the leading part of the SEPR gluon propagator in a covariant gauge is given in Appendix D.

\section{QUARK PROPAGATOR}

\section{A. Preliminaries}

Spin-polarization vector

We define a spin-polarization vector $\mathcal{S}(P)$ as follows. For a timelike $\left(P^{2}=p_{0}^{2}-\vec{p}^{2}>0\right)$ mode, we choose $\mathcal{S}^{\mu}=(0, \vec{\zeta})\left(\equiv \zeta^{\mu}\right)\left[\vec{\zeta}^{2}=1\right]$ in the rest frame, where $P^{\mu}=\left(\epsilon\left(p_{0}\right) \sqrt{P^{2}}, \overrightarrow{0}\right)$. Similarly, for a spacelike $\left(P^{2}<0\right)$ mode, we choose $\mathcal{S}^{\mu}=(0, \vec{\zeta})$ in the " $p_{0}=0$ frame," where $P^{\mu}=\left(0, \sqrt{-P^{2}} \vec{\xi}\right)\left(\equiv \sqrt{-P^{2}} \xi^{\mu}\right)\left[\vec{\xi}^{2}=1, \vec{\xi} \cdot \vec{\zeta}=0\right] . \mathcal{S}(P)$ in any frame, where $P^{\mu}=\left(p_{0}, \vec{p}\right)$, is obtained through a Lorentz transformation:

$$
\begin{aligned}
& \mathcal{S}^{\mu}(P)= \theta\left(P^{2}\right)\left\{\frac{\vec{p} \cdot \vec{\zeta}\left[P^{\mu}+\epsilon\left(p_{0}\right) \sqrt{P^{2}} n^{\mu}\right]}{\sqrt{P^{2}}\left[\sqrt{P^{2}}+\left|p_{0}\right|\right]}+\zeta^{\mu}\right\} \\
&+\theta\left(-P^{2}\right)\left\{-\frac{\vec{p} \cdot \vec{\zeta}\left[P^{\mu}+\epsilon(\vec{p} \cdot \vec{\xi}) \sqrt{-P^{2}} \xi^{\mu}\right]}{\sqrt{-P^{2}}\left[\sqrt{-P^{2}}+|\vec{p} \cdot \vec{\xi}|\right]}+\zeta^{\mu}\right\}, \\
& \mathcal{S} \cdot P=0, \quad \mathcal{S}^{2}=-1, \\
& n^{\mu}=(1, \overrightarrow{0}) .
\end{aligned}
$$

When a magnetic field is applied along the $\vec{\zeta}$ direction, $p_{0}>0$ modes with positive (negative) charge go to the state $\mathcal{S}^{\mu}(P)\left(-\mathcal{S}^{\mu}(P)\right)$, while their "antiparticle" counterparts $\left(p_{0}<0\right.$ modes) go to the state $-\mathcal{S}^{\mu}(P)\left(\mathcal{S}^{\mu}(P)\right)$. In what follows, the concrete form $(2.1)$ is not used, but only the properties (2.2) will be used.

The projection operators $\mathcal{P}_{ \pm}(P)$ onto the states of definite polarization $( \pm)$ reads

$$
\mathcal{P}_{\rho}(P)=\frac{1+\rho \epsilon\left(p_{0}\right) \gamma_{5} \boldsymbol{\phi}(P)}{2}
$$




\section{Orthogonal basis in Minkowski space and the standard form}

As an orthogonal basis in Minkowski space, we choose

$$
\begin{array}{ll}
P^{\mu}, & \mathcal{S}^{\mu}, \\
N^{\mu}=n^{\mu}-\frac{p_{0}}{P^{2}} P^{\mu}+\mathcal{S}_{0} \mathcal{S}^{\mu}, & \left(N^{2}=\mathcal{S}_{0}^{2}-\vec{p}^{2} / P^{2}\right), \\
e_{\perp}^{\mu}=i \epsilon^{\mu \nu \rho \sigma} P_{\nu} N_{\rho} \mathcal{S}_{\sigma}, & \left(e_{\perp}^{2}=-P^{2} N^{2}\right) .
\end{array}
$$

A generic $(4 \times 4)$-matrix function $A\left(P, N, \mathcal{S}, e_{\perp}\right)$ in a Dirac-matrix space is written in the from,

$$
\begin{aligned}
& A=A_{1}^{\prime}+A_{2}^{\prime} \gamma_{5}+A_{3}^{\prime} \not P+A_{4}^{\prime} X^{N}+A_{5}^{\prime} \boldsymbol{\phi}+A_{6}^{\prime} \phi_{\perp} \\
& +A_{7}^{\prime} \gamma_{5} \not P+A_{8}^{\prime} \gamma_{5} \mathcal{X}^{\prime}+A_{9}^{\prime} \gamma_{5} \boldsymbol{\$}+A_{10}^{\prime} \gamma_{5} \phi_{\perp} \\
& +A_{11}^{\prime} \not P D+A_{12}^{\prime} \not P \$+A_{13}^{\prime} \not P \phi_{\perp}+A_{14}^{\prime} X_{X} \phi \\
& +A_{15}^{\prime} \lambda \phi_{\perp}+A_{16}^{\prime} \phi k_{\perp} \text {. }
\end{aligned}
$$

We decompose $A$ into four parts,

$$
A=\sum_{\rho, \sigma= \pm} \mathcal{P}_{\rho} A \mathcal{P}_{\sigma} \equiv \sum_{\rho, \sigma= \pm} \mathcal{P}_{\rho} A^{\rho \sigma} \mathcal{P}_{\sigma}
$$

and write $A^{\rho \sigma}$ in the form,

$$
\begin{aligned}
A^{\rho \rho} & =A_{1}^{\rho \rho}+A_{2}^{\rho \rho} \not P+A_{3}^{\rho \rho} \not \mathcal{N}+A_{4}^{\rho \rho} \not P \mathbb{X}, \\
A^{\rho-\rho} & =\gamma_{5}\left[A_{1}^{\rho-\rho}+A_{2}^{\rho-\rho} \not P+A_{3}^{\rho-\rho} \not \mathcal{N}+A_{4}^{\rho-\rho} \not P \mathbb{N}\right] .
\end{aligned}
$$

It is a straightforward task to obtain

$$
\begin{array}{ll}
A_{1}^{\rho \rho}=A_{1}^{\prime}+\rho \epsilon\left(p_{0}\right) A_{9}^{\prime}, & A_{2}^{\rho \rho}=A_{3}^{\prime}-\rho \epsilon\left(p_{0}\right) N^{2} A_{15}^{\prime}, \\
A_{3}^{\rho \rho}=A_{4}^{\prime}+\rho \epsilon\left(p_{0}\right) P^{2} A_{13}^{\prime}, & A_{4}^{\rho \rho}=A_{11}^{\prime}+\rho \epsilon\left(p_{0}\right) A_{6}^{\prime}, \\
A_{1}^{\rho-\rho}=A_{2}^{\prime}-\rho \epsilon\left(p_{0}\right) A_{5}^{\prime}, & A_{2}^{\rho-\rho}=A_{7}^{\prime}+\rho \epsilon\left(p_{0}\right) A_{12}^{\prime}, \\
A_{3}^{\rho-\rho}=A_{8}^{\prime}+\rho \epsilon\left(p_{0}\right) A_{14}^{\prime}, & A_{4}^{\rho-\rho}=A_{16}^{\prime}-\rho \epsilon\left(p_{0}\right) A_{10}^{\prime} .
\end{array}
$$

We refer Eq. (2.5) to as the standard form $(\mathrm{SF})$ and $A^{\rho \sigma}$ or $A_{j}^{\rho \sigma}$ to as an SF-element of $A$. It is to be understood that the (bare and self-energy-part resummed) propagators and the self-energy part, which appear in the following, are to be written in the SF. 


\section{B. Bare propagator}

First of all, we note that the bare propagator matrix $\hat{S}(P)$ and the self-energy-part resummed propagator matrix $\hat{G}(P)$ enjoy the "symmetry" property,

$$
\hat{S}^{\dagger}(P)=-\hat{\tau}_{1} \gamma^{0}{ }^{\mathrm{t}} \hat{S}(P) \gamma^{0} \hat{\tau}_{1}, \quad \hat{G}^{\dagger}(P)=-\hat{\tau}_{1} \gamma^{0}{ }^{\mathrm{t}} \hat{G}(P) \gamma^{0} \hat{\tau}_{1}
$$

which results from the hermiticity of the density matrix. Here, $\hat{\tau}_{1}$ is the first Pauli matrix, $\dagger$ acts on Dirac gamma matrix function, e.g., $(A \not P)^{\dagger}=A^{*} P^{\mu} \gamma_{\mu}^{\dagger}$, and ${ }^{\mathrm{t}} \hat{S}(P)$ denotes the transpose of the $(2 \times 2)$ matrix function $\hat{S}(P)$, etc.

The bare propagator $\hat{S}(P)$ is an inverse of $\hat{S}^{-1}(P)=(\not P-m) \hat{\tau}_{3}$. A general solution to $\hat{S}^{-1} \hat{S}=\hat{S} \hat{S}^{-1}=1$ is

$$
\begin{aligned}
\hat{S}(P)= & \hat{S}^{(0)}(P)+S_{K}(P) \hat{M}_{+}, \\
\hat{S}^{(0)}(P)= & \sum_{\rho= \pm} \mathcal{P}_{\rho}\left[\hat{S}_{R A}(P)-f_{\rho}\left(S_{R}-S_{A}\right) \hat{M}_{+}\right], \\
S_{K}(P)= & -\sum_{\rho= \pm} C_{\rho-\rho}(P)\left(\Delta_{R}(P)-\Delta_{A}(P)\right) \\
& \times \mathcal{P}_{\rho} \gamma_{5}(\not P-m) \not N \mathcal{P}_{-\rho},
\end{aligned}
$$

where the suffix " $K$ " stands for the "Keldish component" and

$$
\begin{aligned}
\hat{S}_{R A}(P)= & \left(\begin{array}{cc}
S_{R} & 0 \\
S_{R}-S_{A} & -S_{A}
\end{array}\right), \\
\hat{M}_{ \pm}= & \left(\begin{array}{cc}
1 & \pm 1 \\
\pm 1 & 1
\end{array}\right), \\
S_{R(A)}= & (\not P+m) \Delta_{R(A)}(P)=\frac{\not P+m}{P^{2}-m^{2} \pm i p_{0} 0^{+}}, \\
f_{\rho}(P)= & \theta\left(p_{0}\right) N_{\rho}\left(\left|p_{0}\right|, \vec{p}\right) \\
& +\theta\left(-p_{0}\right)\left[1-\bar{N}_{\rho}\left(\left|p_{0}\right|,-\vec{p}\right)\right] .
\end{aligned}
$$

Here $S_{R(A)}$ is the retarded (advanced) propagator, and $N_{\rho}\left(\left|p_{0}\right|, \vec{p}\right)\left[\bar{N}_{\rho}\left(\left|p_{0}\right|,-\vec{p}\right)\right](\rho= \pm)$ is the "bare" number-density function of a quark [an antiquark] with polarization $\rho \mathcal{S}(P)$, 
energy $\left|p_{0}\right|\left(=\sqrt{\vec{p}^{2}+m^{2}}\right)$, and momentum $\vec{p}[-\vec{p}] . \quad S_{K}$ in Eq. (2.10) connects opposite polarization states. From Eqs. (2.7), (2.8), and (2.10), we have

$$
\left(C_{+-}(P)\right)^{*}=C_{-+}(P)
$$

The derivative expansion is an efficient device for dealing with quasiuniform systems near equilibrium or nonequilibrium quasistationary systems. For such systems, $S_{K}$ is small when compared to $\hat{S}^{(0)}$.

\section{Dyson equation}

The self-energy-part $(\hat{\Sigma})$ resummed propagator $\hat{G}$ obeys the Dyson equation,

$$
\hat{G}(P)=\hat{S}(P)[1+\hat{\Sigma}(P) \hat{G}(P)]=[1+\hat{G}(P) \hat{\Sigma}(P)] \hat{S}(P) .
$$

We write $\hat{G}$ and $\hat{\Sigma}$ in SFs,

$$
\hat{G}=\sum_{\rho, \sigma= \pm} \mathcal{P}_{\rho} \hat{G}^{\rho \sigma} \mathcal{P}_{\sigma}, \quad \hat{\Sigma}=\sum_{\rho, \sigma= \pm} \mathcal{P}_{\rho} \hat{\Sigma}^{\rho \sigma} \mathcal{P}_{\sigma}
$$

It is worth mentioning that, for the system that enjoys an azimuthal symmetry around

the $\vec{\zeta}$-direction, $\hat{\Sigma}$, and then also $\hat{G}$, are independent of $E_{\perp}^{\mu}$, provided that we choose $\vec{\xi}=$ $\vec{p} \times \vec{\zeta} /|\vec{p} \times \vec{\zeta}|$. Then, from Eqs. (2.3) - (2.6), we have

$$
\begin{array}{ll}
\hat{\Sigma}_{4}^{\rho-\rho}=0 & (\rho= \pm), \\
\hat{\Sigma}_{j}^{++}=\hat{\Sigma}_{j}^{--} & (j=2,3,4) .
\end{array}
$$

Same relations hold for $\hat{G}^{\prime}$ s.

Substituting the SFs for $\hat{S}, \hat{\Sigma}$, and $\hat{G}$ in Eq. (2.13), we obtain coupled equations,

$$
\begin{aligned}
\hat{G}^{\rho \sigma}=\hat{S}^{\rho \sigma}+(\hat{S} \hat{\Sigma} \hat{G})^{\rho \sigma}=\hat{S}^{\rho \sigma}+(\hat{G} \hat{\Sigma} \hat{S})^{\rho \sigma} & \\
& (\rho, \sigma= \pm)
\end{aligned}
$$

where $(\hat{S} \hat{\Sigma} \hat{G})^{\rho \sigma} \equiv \sum_{\xi, \zeta= \pm} \hat{S}^{\rho \xi} \hat{\Sigma}^{\xi \zeta} \hat{G}^{\zeta \sigma}$, etc. The relation that involves $(\ldots)^{\rho \sigma}$ is to be understood to hold when sandwiched between projection operators $\mathcal{P}_{\rho} \ldots \mathcal{P}_{\sigma}$. We write Eq. (2.14), with obvious notation, as 


$$
\hat{\mathbf{G}}=\hat{\mathbf{S}}+\hat{\mathbf{S}} \hat{\Sigma} \hat{\mathbf{G}}=\hat{\mathbf{S}}+\hat{\mathbf{G}} \hat{\Sigma} \hat{\mathbf{S}}
$$

where bold-face letters denote $(2 \times 2)$-matrix in a "polarization space".

From Eq. (2.7), we obtain the symmetry relations for the SF-elements of $\hat{G}^{\rho \sigma}$ (cf. Eqs. (2.4) and (2.5)),

$$
\left(\hat{G}_{j}^{\rho \sigma}(P)\right)^{*}=-\sigma_{j}^{\rho \sigma} \hat{\tau}_{1}{ }^{\mathrm{t}} \hat{G}_{j}^{\sigma \rho}(P) \hat{\tau}_{1},
$$

where

$$
\sigma_{j}^{\rho \sigma}=\left\{\begin{aligned}
+ \text { for }(\rho \sigma, j)= & (\rho \rho, 1),(\rho \rho, 2),(\rho \rho, 3), \\
& (\rho-\rho, 2),(\rho-\rho, 3), \\
& (\rho-\rho, 4), \\
- \text { for }(\rho \sigma, j)= & (\rho \rho, 4),(\rho-\rho, 1) .
\end{aligned}\right.
$$

Similar relations hold for $\hat{\Sigma}_{j}^{\rho \sigma}$ s.

Let us introduce $(2 \times 2)$-matrix function $\mathbf{f}$ in the polarization space,

$$
\mathbf{f}=\operatorname{diag}\left(f_{+}, f_{-}\right) .
$$

Then $\hat{\mathbf{S}}$ is written as

$$
\begin{aligned}
\hat{\mathbf{S}} & =\hat{\mathbf{S}}^{(0)}+\mathbf{S}_{K} \hat{M}_{+}, \\
\hat{\mathbf{S}}^{(0)} & =\hat{S}_{R A} \mathbf{1}-\mathbf{f}\left(S_{R}-S_{A}\right) \hat{M}_{+} \\
\mathbf{S}_{K} & =\left(S_{R}(P)-S_{A}(P)\right) \gamma_{5} N^{\prime} \mathbf{C}(P), \\
\mathbf{C}(P) & =\left(\begin{array}{cc}
0 & C_{+-}(P) \\
C_{-+}(P) & 0
\end{array}\right) .
\end{aligned}
$$

Among the components of $\hat{\boldsymbol{\Sigma}}$, is a relation,

$$
\Sigma_{11}+\Sigma_{12}+\Sigma_{21}+\Sigma_{22}=0 .
$$

Then, $\hat{\Sigma}$ is written as 


$$
\begin{aligned}
& \hat{\Sigma}=\hat{\Sigma}^{(0)}-\Sigma_{K} \hat{M}_{-}, \\
& \hat{\Sigma}^{(0)}=\left(\begin{array}{cc}
\boldsymbol{\Sigma}_{R} & 0 \\
-\boldsymbol{\Sigma}_{R}+\boldsymbol{\Sigma}_{A} & -\boldsymbol{\Sigma}_{A}
\end{array}\right)-\left(\boldsymbol{\Sigma}_{R} \mathbf{f}-\mathbf{f} \boldsymbol{\Sigma}_{A}\right) \hat{M}_{-}, \\
& \Sigma_{R}=\Sigma_{11}+\Sigma_{12}=-\Sigma_{22}-\Sigma_{21} \\
& \Sigma_{A}=\Sigma_{11}+\Sigma_{21}=-\Sigma_{22}-\Sigma_{12}, \\
& \Sigma_{K}=\mathbf{f} \Sigma_{11}-\Sigma_{11} \mathbf{f}+\mathbf{f} \Sigma_{21}+\Sigma_{12}(1-\mathbf{f}) .
\end{aligned}
$$

From Eq. (2.16) with $\hat{\Sigma}_{j}^{\rho \sigma}$ for $\hat{G}_{j}^{\rho \sigma}$, we obtain the symmetry relations,

$$
\begin{aligned}
& \left(\Sigma_{R j}^{\rho \sigma}(P)\right)^{*}=\sigma_{j}^{\rho \sigma} \Sigma_{A j}^{\sigma \rho}(P), \\
& \left(\Sigma_{K j}^{\rho \sigma}(P)\right)^{*}=-\sigma_{j}^{\rho \sigma} \Sigma_{K j}^{\sigma \rho}(P) .
\end{aligned}
$$

Among the components of $\hat{\mathbf{G}}$, is a relation,

$$
\mathbf{G}_{11}+\mathbf{G}_{22}=\mathbf{G}_{12}+\mathbf{G}_{21}
$$

Then, $\hat{\mathbf{G}}$ is written as

$$
\begin{aligned}
\hat{\mathbf{G}} & =\hat{\mathbf{G}}^{(0)}+\mathbf{G}_{K} \hat{M}_{+}, \\
\hat{\mathbf{G}}^{(0)} & =\left(\begin{array}{cc}
\mathbf{G}_{R} & 0 \\
\mathbf{G}_{R}-\mathbf{G}_{A} & -\mathbf{G}_{A}
\end{array}\right)-\left(\mathbf{G}_{R} \mathbf{f}-\mathbf{f G}_{A}\right) \hat{M}_{+}, \\
\mathbf{G}_{R} & =\mathbf{G}_{11}-\mathbf{G}_{12}=-\mathbf{G}_{22}+\mathbf{G}_{21}, \\
\mathbf{G}_{A} & =\mathbf{G}_{11}-\mathbf{G}_{21}=-\mathbf{G}_{22}+\mathbf{G}_{12}, \\
\mathbf{G}_{K} & =\mathbf{G}_{11} \mathbf{f}-\mathbf{f G}_{11}+\mathbf{f G}_{21}+\mathbf{G}_{12}(1-\mathbf{f}) .
\end{aligned}
$$

It is worth mentioning that, for equilibrium systems, $\boldsymbol{\Sigma}_{K}=\mathbf{G}_{K}=0$. From Eq. (2.16), follows the symmetry relations,

$$
\begin{aligned}
& \left(G_{R j}^{\rho \sigma}(P)\right)^{*}=\sigma_{j}^{\rho \sigma} G_{A j}^{\sigma \rho}(P), \\
& \left(G_{K j}^{\rho \sigma}(P)\right)^{*}=-\sigma_{j}^{\rho \sigma} G_{K j}^{\sigma \rho}(P) .
\end{aligned}
$$


Substitution of Eqs. (2.17), (2.20), and (2.26) into Eq. (2.15) yields

$$
\begin{aligned}
\hat{\mathbf{G}}^{(0)}= & \hat{\mathbf{S}}^{(0)}+\hat{\mathbf{S}}^{(0)} \hat{\boldsymbol{\Sigma}}^{(0)} \hat{\mathbf{G}}^{(0)}=\hat{\mathbf{S}}^{(0)}+\hat{\mathbf{G}}^{(0)} \hat{\boldsymbol{\Sigma}}^{(0)} \hat{\mathbf{S}}^{(0)} \\
\mathbf{G}_{K}= & \mathbf{S}_{K}+S_{R} \boldsymbol{\Sigma}_{R} \mathbf{G}_{K}+\mathbf{S}_{K} \boldsymbol{\Sigma}_{A} \mathbf{G}_{A}-S_{R} \boldsymbol{\Sigma}_{K} \mathbf{G}_{A} \\
& =\mathbf{S}_{K}+\mathbf{G}_{R} \boldsymbol{\Sigma}_{R} \mathbf{S}_{K}+\mathbf{G}_{K} \boldsymbol{\Sigma}_{A} S_{A}-\mathbf{G}_{R} \boldsymbol{\Sigma}_{K} S_{A}
\end{aligned}
$$

From Eq. (2.32), we obtain

$$
\mathbf{G}_{R(A)}=\left[\not P-m-\boldsymbol{\Sigma}_{R(A)}\right]^{-1}
$$

where use has been made of $\left(\hat{S}^{(0)-1}\right)^{\rho \sigma}=\delta^{\rho \sigma}(\not P-m) \hat{\tau}_{3}$. We get from Eq. (2.34), after some manipulation,

$$
\begin{gathered}
G_{R}^{\rho \rho}=\left[\not P-m-\Sigma_{R}^{\rho \rho}-\Sigma_{R}^{\rho-\rho} G_{R}^{(\mathrm{pre})-\rho-\rho} \Sigma_{R}^{-\rho \rho}\right]^{-1}, \\
G_{R}^{\rho-\rho}=G_{R}^{(\mathrm{pre}) \rho \rho} \Sigma_{R}^{\rho-\rho} G_{R}^{-\rho-\rho}=G_{R}^{\rho \rho} \Sigma_{R}^{\rho-\rho} G_{R}^{(\mathrm{pre})-\rho-\rho},
\end{gathered}
$$

where

$$
G_{R}^{(\mathrm{pre}) \rho \rho}=\left[\not P-m-\Sigma_{R}^{\rho \rho}\right]^{-1}
$$

As has been remarked above after Eq. (2.14), Eq. (2.37) is to be understood to mean

$$
\begin{aligned}
\mathcal{P}_{\rho} G_{R}^{(\mathrm{pre}) \rho \rho}\left[\not P-m-\Sigma_{R}^{\rho \rho}\right] \mathcal{P}_{\rho} \\
\quad=\mathcal{P}_{\rho}\left[\not P-m-\Sigma_{R}^{\rho \rho}\right] G_{R}^{(\mathrm{pre}) \rho \rho} \mathcal{P}_{\rho}=\mathcal{P}_{\rho} 1 \mathcal{P}_{\rho}=\mathcal{P}_{\rho}
\end{aligned}
$$

Such an understanding also applies to Eq. (2.35). Concrete form for $\mathbf{G}_{R(A)}$ will be given in the next section. 
As for $\mathbf{G}_{K}$, Eq. (2.33), we show in Appendix A that

$$
\begin{aligned}
\mathbf{G}_{K} & =\mathbf{G}_{K}^{(1)}+\mathbf{G}_{K}^{(2)}+\mathbf{G}_{K}^{(3)} \\
\mathbf{G}_{K}^{(1)} & =-\mathbf{G}_{R} \boldsymbol{\Sigma}_{K} \mathbf{G}_{A}, \\
\mathbf{G}_{K}^{(2)} & =\mathbf{G}_{R}\left[\gamma_{5} \perp \mathbf{C}(P) \boldsymbol{\Sigma}_{A}-\boldsymbol{\Sigma}_{R} \gamma_{5} N \mathbf{C}(P)\right] \mathbf{G}_{A} \\
& \equiv \mathbf{G}_{R} \mathbf{H}_{l} \mathbf{G}_{A}, \\
\mathbf{G}_{K}^{(3)} & =\mathbf{G}_{R} \gamma_{5} \perp \mathbf{C}(P)-\gamma_{5} N \mathbf{C}(P) \mathbf{G}_{A} .
\end{aligned}
$$

"l" of $\mathbf{H}_{l}$ in Eq. (2.41) stands for the "leading part" of the DEX. As mentioned above at the end of Subsec. B, for quasiuniform systems near equilibrium or nonequilibrium

quasistationary systems, $\mathbf{G}_{K}^{(2)}$ and $\mathbf{G}_{K}^{(3)}$ are much smaller than $\mathbf{G}_{K}^{(1)}$. The $\mathrm{SF}$ for $\mathbf{G}_{K}^{(1)}$ will be given in the next section. The standard forms for $\mathbf{H}_{l}$ in Eq. (2.41) and $\gamma_{5} X / \mathbf{C}(P)$ in Eq. (2.42) are also given in the next section. The SFs for $\mathbf{G}_{K}^{(2)}$ and $\mathbf{G}_{K}^{(3)}$ are obtained by repeatedly using the formulae in Appendix B.

\section{Self-energy-part resummed propagator $\hat{\mathbf{G}}$}

It is convenient to introduce

$$
\hat{\tilde{G}}^{\rho \rho}=\left(\begin{array}{cc}
G_{R}^{\rho \rho} & G_{K}^{(1) \rho \rho} \\
0 & -G_{A}^{\rho \rho}
\end{array}\right), \quad \hat{\tilde{\Sigma}}^{\rho \sigma}=\left(\begin{array}{cc}
\Sigma_{R}^{\rho \sigma} & \Sigma_{K}^{\rho \sigma} \\
0 & -\Sigma_{A}^{\rho \sigma}
\end{array}\right) .
$$

We observe that Eq. (2.35) and $G_{K}^{(1) \rho \rho}$ in Eq. (2.40) are unified to a matrix equation,

$$
\hat{\tilde{G}}^{\rho \rho}=\left[(\not P-m) \hat{\tau}_{3}-\hat{\tilde{\Sigma}}^{\rho \rho}-\hat{\tilde{\Sigma}}^{\rho-\rho} \hat{\tilde{G}}^{(\mathrm{pre})-\rho-\rho} \hat{\tilde{\Sigma}}^{-\rho \rho}\right]^{-1}
$$

where

$$
\begin{aligned}
\hat{\tilde{G}}^{(\mathrm{pre}) \rho \rho} & =\left(\begin{array}{cc}
G_{R}^{(\mathrm{pre}) \rho \rho} & G_{K}^{(\mathrm{pre}) \rho \rho} \\
0 & -G_{A}^{(\mathrm{pre}) \rho \rho}
\end{array}\right) \\
& =\left[(\not P-m) \hat{\tau}_{3}-\hat{\tilde{\Sigma}}^{\rho \rho}\right]^{-1}
\end{aligned}
$$




$$
\text { Forms for } G_{R(A)}^{(\mathrm{pre}) \rho \rho} \text { and } G_{K}^{(\mathrm{pre}) \rho \rho}
$$

The SF for $(\not P-m) \hat{\tau}_{3}-\hat{\tilde{\Sigma}}^{\rho \rho}$ reads

$$
\begin{aligned}
(\not P-m) \hat{\tau}_{3}-\hat{\tilde{\Sigma}}^{\rho \rho}(P) & \\
= & -\left[m \hat{\tau}_{3}+\hat{\tilde{\Sigma}}_{1}^{\rho \rho}(P)\right]+\left[\hat{\tau}_{3}-\hat{\tilde{\Sigma}}_{2}^{\rho \rho}(P)\right] \not P \\
& -\hat{\tilde{\Sigma}}_{3}^{\rho \rho}(P) \mathcal{N}-\hat{\tilde{\Sigma}}_{4}^{\rho \rho}(P) \not P X
\end{aligned}
$$

One obtains the expressions for the SF-elements $G_{T j}^{(\text {pre }) \rho \rho}(T=R, A, K$ and $j=1-4)$ through straightforward but tedious manipulation of Eq. (2.44), which includes Eq. (2.38). Writing $\Sigma_{j} \equiv \Sigma_{R j}^{\rho \rho}$ for short, we have

$$
\begin{aligned}
& G_{R j}^{(\text {pre }) \rho \rho}=\sigma_{j}^{\rho \rho}\left(G_{A j}^{(\text {pre }) \rho \rho}\right)^{*} \quad(j=1-4), \\
& G_{R 1}^{(\text {pre }) \rho \rho}=\frac{m+\Sigma_{1}}{\mathcal{D}_{\text {pre }}^{\rho \rho}}, \quad G_{R 2}^{(\text {pre }) \rho \rho}=\frac{1-\Sigma_{2}}{\mathcal{D}_{\text {pre }}^{\rho \rho}}, \\
& G_{R l}^{(\text {pre }) \rho \rho}=-\frac{\Sigma_{l}}{\mathcal{D}_{\text {pre }}^{\rho \rho}} \quad(l=3,4), \\
& G_{K j}^{(\text {pre }) \rho \rho}=\frac{\sum_{l=1}^{4} \mathcal{N}_{j}^{(l)} \Sigma_{K l}^{\rho \rho}}{I m\left\{\left[\left(m+\Sigma_{1}\right)^{2}-N^{2}\left(\Sigma_{3}\right)^{2}\right]\left[\left(\Sigma_{2}^{*}-1\right)^{2}-N^{2}\left(\Sigma_{4}^{*}\right)^{2}\right]\right\}} \quad(j=1-4),
\end{aligned}
$$

where

$$
\mathcal{D}_{\text {pre }}^{\rho \rho}=\left[\left(1-\Sigma_{2}\right)^{2}-N^{2}\left(\Sigma_{4}\right)^{2}\right] P^{2}-\left(m+\Sigma_{1}\right)^{2}+N^{2}\left(\Sigma_{3}\right)^{2}
$$

and

$$
\begin{array}{lll}
\mathcal{N}_{1}^{(1)}=-\left[E_{13}^{(+)} F_{24}+E_{24}^{(+)} F_{13}\right], & \mathcal{N}_{1}^{(2)}=-2 F_{13} R e F_{1234}^{(-)}, \\
\mathcal{N}_{1}^{(3)}=2 N^{2} \operatorname{Re}\left[F_{13} H_{24}+F_{24} H_{13}\right], & \mathcal{N}_{1}^{(4)}=2 i N^{2} F_{13} I m F_{1423}^{(+)}, \\
\mathcal{N}_{2}^{(1)}=-2 F_{24} \operatorname{Re} F_{1234}^{(+)}, & \mathcal{N}_{2}^{(2)}=-\left[E_{24}^{(-)} F_{13}+E_{13}^{(-)} F_{24}\right], \\
\mathcal{N}_{2}^{(3)}=2 N^{2} F_{24} R e F_{1423}^{(+)}, & \mathcal{N}_{2}^{(4)}=2 i N^{2} \operatorname{Im}\left[F_{13} H_{24}+F_{24} H_{13}\right], \\
\mathcal{N}_{3}^{(1)}=-2 \operatorname{Re}\left[F_{13} H_{24}-F_{24} H_{13}\right], & \mathcal{N}_{3}^{(2)}=-2 F_{13} R e F_{1423}^{(-)}, \\
\mathcal{N}_{3}^{(3)}=-\left[E_{13}^{(+)} F_{24}-E_{24}^{(+)} F_{13}\right], & \mathcal{N}_{3}^{(4)}=2 i F_{13} I m F_{1234}^{(-)}, \\
\mathcal{N}_{4}^{(1)}=-2 i F_{24} \operatorname{Im} F_{1423}^{(-)}, & \mathcal{N}_{4}^{(2)}=2 i \operatorname{Im}\left[-F_{13} H_{24}+F_{24} H_{13}\right] \\
\mathcal{N}_{4}^{(3)}=2 i F_{24} \operatorname{Im} F_{1234}^{(+)}, & \mathcal{N}_{4}^{(4)}=-\left[E_{13}^{(-)} F_{24}-E_{24}^{(-)} F_{13}\right],
\end{array}
$$


with

$$
\begin{array}{ll}
E_{13}^{( \pm)}=\left|m+\Sigma_{1}\right|^{2} \pm N^{2}\left|\Sigma_{3}\right|^{2}, & E_{24}^{( \pm)}=\left|1-\Sigma_{2}\right|^{2} \pm N^{2}\left|\Sigma_{4}\right|^{2}, \\
F_{24}=\operatorname{Im} \frac{\left(1-\Sigma_{2}\right)^{2}-N^{2}\left(\Sigma_{4}\right)^{2}}{\mathcal{D}_{\operatorname{pre}}^{\rho \rho}}, & F_{13}=\operatorname{Im} \frac{\left(m+\Sigma_{1}\right)^{2}-N^{2}\left(\Sigma_{3}\right)^{2}}{\mathcal{D}_{\mathrm{pre}}^{\rho \rho}} \\
F_{1234}^{( \pm)}=\left(m+\Sigma_{1}\right)\left(1-\Sigma_{2}^{*}\right) \pm N^{2} \Sigma_{3} \Sigma_{4}^{*}, & \\
F_{1423}^{( \pm)}=\left(m+\Sigma_{1}\right) \Sigma_{4}^{*} \pm\left(1-\Sigma_{2}\right) \Sigma_{3}^{*}, & \\
H_{13}=\left(m+\Sigma_{1}\right) \Sigma_{3}^{*}, & H_{24}=\left(1-\Sigma_{2}\right) \Sigma_{4}^{*} .
\end{array}
$$

Form for $G_{R(A)}^{\rho \rho}$ and $G_{K}^{(1) \rho \rho}$, Eqs. (2.35) and (2.40)

Using the definition (B.1) in Appendix B, one can write the quantity in the square brackets in Eq. (2.43) as

$$
(\not P-m) \hat{\tau}_{3}-\hat{\tilde{\Sigma}}^{\rho \rho}-\left[\left[\hat{\tilde{\Sigma}} \otimes \hat{\tilde{G}}^{(\mathrm{pre})}\right] \otimes \hat{\tilde{\Sigma}}\right]^{\rho \rho} .
$$

The SF for this is obtained by the repeated use of the formulae in Appendix B:

$$
\begin{aligned}
\text { Eq. (2.47) }= & -\left[m \hat{\tau}_{3}+\hat{\tilde{\Sigma}}_{1}^{\rho \rho}+\hat{A}_{1}^{\rho-\rho} \hat{\tilde{\Sigma}}_{1}^{-\rho \rho}-P^{2} \hat{A}_{2}^{\rho-\rho} \hat{\tilde{\Sigma}}_{2}^{-\rho \rho}-N^{2} \hat{A}_{3}^{\rho-\rho} \hat{\tilde{\Sigma}}_{3}^{-\rho \rho}-P^{2} N^{2} \hat{A}_{4}^{\rho-\rho} \hat{\tilde{\Sigma}}_{4}^{-\rho \rho}\right] \\
& +\left[\hat{\tau}_{3}-\hat{\tilde{\Sigma}}_{2}^{\rho \rho}-\hat{A}_{1}^{\rho-\rho} \hat{\tilde{\Sigma}}_{2}^{-\rho \rho}+\hat{A}_{2}^{\rho-\rho} \hat{\tilde{\Sigma}}_{1}^{-\rho \rho}-N^{2} \hat{A}_{3}^{\rho-\rho} \hat{\tilde{\Sigma}}_{4}^{-\rho \rho}-N^{2} \hat{A}_{4}^{\rho-\rho} \hat{\tilde{\Sigma}}_{3}^{-\rho \rho}\right] \not P \\
& -\left[\hat{\tilde{\Sigma}}_{3}^{\rho \rho}+\hat{A}_{1}^{\rho-\rho} \hat{\tilde{\Sigma}}_{3}^{-\rho \rho}-P^{2} \hat{A}_{2}^{\rho-\rho} \hat{\tilde{\Sigma}}_{4}^{-\rho \rho}-\hat{A}_{3}^{\rho-\rho} \hat{\tilde{\Sigma}}_{1}^{-\rho \rho}-P^{2} \hat{A}_{4}^{\rho-\rho} \hat{\tilde{\Sigma}}_{2}^{-\rho \rho}\right] X^{\top} \\
& -\left[\hat{\tilde{\Sigma}}_{4}^{\rho \rho}+\hat{A}_{1}^{\rho-\rho} \hat{\tilde{\Sigma}}_{4}^{-\rho \rho}-\hat{A}_{2}^{\rho-\rho} \hat{\tilde{\Sigma}}_{3}^{-\rho \rho}+\hat{A}_{3}^{\rho-\rho} \hat{\tilde{\Sigma}}_{2}^{-\rho \rho}+\hat{A}_{4}^{\rho-\rho} \hat{\tilde{\Sigma}}_{1}^{-\rho \rho}\right] \not P N
\end{aligned}
$$

where

$$
\begin{aligned}
& \hat{A}_{1}^{\rho-\rho}=\hat{\tilde{\Sigma}}_{1}^{\rho-\rho} \hat{\tilde{G}}_{1}^{(\text {pre })-\rho-\rho}+P^{2} \hat{\tilde{\Sigma}}_{2}^{\rho-\rho} \hat{\tilde{G}}_{2}^{(\text {pre })-\rho-\rho}+N^{2} \hat{\tilde{\Sigma}}_{3}^{\rho-\rho} \hat{\tilde{G}}_{3}^{(\text {pre })-\rho-\rho}-P^{2} N^{2} \hat{\tilde{\Sigma}}_{4}^{\rho-\rho} \hat{\tilde{G}}_{4}^{(\text {pre })-\rho-\rho} \\
& \hat{A}_{2}^{\rho-\rho}=\hat{\tilde{\Sigma}}_{1}^{\rho-\rho} \hat{\tilde{G}}_{2}^{(\text {pre })-\rho-\rho}+\hat{\tilde{\Sigma}}_{2}^{\rho-\rho} \hat{\tilde{G}}_{1}^{(\text {pre })-\rho-\rho}-N^{2} \hat{\tilde{\Sigma}}_{3}^{\rho-\rho} \hat{\tilde{G}}_{4}^{(\text {pre })-\rho-\rho}+N^{2} \hat{\tilde{\Sigma}}_{4}^{\rho-\rho} \hat{\tilde{G}}_{3}^{(\text {pre })-\rho-\rho}, \\
& \hat{A}_{3}^{\rho-\rho}=\hat{\tilde{\Sigma}}_{1}^{\rho-\rho} \hat{\tilde{G}}_{3}^{(\text {pre })-\rho-\rho}+P^{2} \hat{\tilde{\Sigma}}_{2}^{\rho-\rho} \hat{\tilde{G}}_{4}^{(\text {pre })-\rho-\rho}+\hat{\tilde{\Sigma}}_{3}^{\rho-\rho} \hat{\tilde{G}}_{1}^{(\text {pre })-\rho-\rho}-P^{2} \hat{\tilde{\Sigma}}_{4}^{\rho-\rho} \hat{\tilde{G}}_{2}^{(\text {pre })-\rho-\rho}, \\
& \hat{A}_{4}^{\rho-\rho}=\hat{\tilde{\Sigma}}_{1}^{\rho-\rho} \hat{\tilde{G}}_{4}^{(\text {pre })-\rho-\rho}+\hat{\tilde{\Sigma}}_{2}^{\rho-\rho} \hat{\tilde{G}}_{3}^{(\text {pre })-\rho-\rho}-\hat{\tilde{\Sigma}}_{3}^{\rho-\rho} \hat{\tilde{G}}_{2}^{(\text {pre })-\rho-\rho}+\hat{\tilde{\Sigma}}_{4}^{\rho-\rho} \hat{\tilde{G}}_{1}^{(\text {pre })-\rho-\rho} .
\end{aligned}
$$

We observe that Eq. (2.48) with Eq. (2.49) is obtained from Eq. (2.45) through the following substitutions ( $T$ stands for $R, A$, or $K$ ), 


$$
\begin{aligned}
\Sigma_{T 1}^{\rho \rho} \rightarrow & \Sigma_{T 1}^{\rho \rho}+T_{111}^{\rho \rho}+P^{2} T_{221}^{\rho \rho}+N^{2} T_{331}^{\rho \rho}-P^{2} N^{2} T_{441}^{\rho \rho}-P^{2}\left[T_{122}^{\rho \rho}+T_{212}^{\rho \rho}-N^{2} T_{342}^{\rho \rho}+N^{2} T_{432}^{\rho \rho}\right] \\
& -N^{2}\left[T_{133}^{\rho \rho}+P^{2} T_{243}^{\rho \rho}+T_{313}^{\rho \rho}-P^{2} T_{423}^{\rho \rho}\right]-P^{2} N^{2}\left[T_{144}^{\rho \rho}+T_{234}^{\rho \rho}-T_{324}^{\rho \rho}+T_{414}^{\rho \rho}\right] \\
\Sigma_{T 2}^{\rho \rho} \rightarrow & \Sigma_{T 2}^{\rho \rho}+T_{112}^{\rho \rho}+P^{2} T_{222}^{\rho \rho}+N^{2} T_{332}^{\rho \rho}-P^{2} N^{2} T_{442}^{\rho \rho}-T_{121}^{\rho \rho}-T_{211}^{\rho \rho}+N^{2} T_{341}^{\rho \rho}-N^{2} T_{431}^{\rho \rho} \\
& +N^{2}\left[T_{134}^{\rho \rho}+P^{2} T_{244}^{\rho \rho}+T_{314}^{\rho \rho}-P^{2} T_{424}^{\rho \rho}\right]+N^{2}\left[T_{143}^{\rho \rho}+T_{233}^{\rho \rho}-T_{323}^{\rho \rho}+T_{413}^{\rho \rho}\right] \\
\Sigma_{T 3}^{\rho \rho} \rightarrow & \Sigma_{T 3}^{\rho \rho}+T_{113}^{\rho \rho}+P^{2} T_{223}^{\rho \rho}+N^{2} T_{333}^{\rho \rho}-P^{2} N^{2} T_{443}^{\rho \rho}-P^{2}\left[T_{124}^{\rho \rho}+T_{214}^{\rho \rho}-N^{2} T_{344}^{\rho \rho}+N^{2} T_{434}^{\rho \rho}\right] \\
& -\left[T_{131}^{\rho \rho}+P^{2} T_{241}^{\rho \rho}+T_{311}^{\rho \rho}-P^{2} T_{421}^{\rho \rho}\right]-P^{2}\left[T_{142}^{\rho \rho}+T_{232}^{\rho \rho}-T_{322}^{\rho \rho}+T_{412}^{\rho \rho}\right] \\
\Sigma_{T 4}^{\rho \rho} \rightarrow & \Sigma_{T 4}^{\rho \rho}+T_{114}^{\rho \rho}+P^{2} T_{224}^{\rho \rho}+N^{2} T_{334}^{\rho \rho}-P^{2} N^{2} T_{444}^{\rho \rho}-\left[T_{123}^{\rho \rho}+T_{213}^{\rho \rho}-N^{2} T_{343}^{\rho \rho}+N^{2} T_{433}^{\rho \rho}\right] \\
& +\left[T_{132}^{\rho \rho}+P^{2} T_{242}^{\rho \rho}+T_{312}^{\rho \rho}-P^{2} T_{422}^{\rho \rho}\right]+\left[T_{141}^{\rho \rho}+T_{231}^{\rho \rho}-T_{321}^{\rho \rho}+T_{411}^{\rho \rho}\right] .
\end{aligned}
$$

Here, for $T=R$ and $A$,

$$
R_{i j l}^{\rho \rho}=\Sigma_{R i}^{\rho-\rho} G_{R j}^{(\mathrm{pre})-\rho-\rho} \Sigma_{R l}^{-\rho \rho}
$$

and

$$
A_{i j l}^{\rho \rho}=\Sigma_{A i}^{\rho-\rho} G_{A j}^{(\mathrm{pre})-\rho-\rho} \Sigma_{A l}^{-\rho \rho},
$$

respectively, and, for $T=K$,

$$
\begin{aligned}
K_{i j l}^{\rho \rho}= & \Sigma_{R i}^{\rho-\rho}\left[G_{R j}^{(\mathrm{pre})-\rho-\rho} \Sigma_{K l}^{-\rho \rho}-G_{K j}^{(\mathrm{pre})-\rho-\rho} \Sigma_{A l}^{-\rho \rho}\right] \\
& +\Sigma_{K i}^{\rho-\rho} G_{A j}^{(\mathrm{pre})-\rho-\rho} \Sigma_{A l}^{-\rho \rho} .
\end{aligned}
$$

Then, the expressions for $G_{R j}^{\rho \rho}, G_{A j}^{\rho \rho}$, and $G_{K j}^{(1) \rho \rho}(j=1-4)$ are obtained from those of their counterparts, in respective order, $G_{R j}^{(\mathrm{pre}) \rho \rho}, G_{A j}^{(\mathrm{pre}) \rho \rho}$, and $G_{K j}^{(\mathrm{pre}) \rho \rho}$ with the above substitutions.

The forms for $H_{l}^{\rho \sigma}$ in $G_{K}^{(2) \rho \sigma}$, Eq. (2.41), and for $\gamma_{5} \not{ }^{\dagger} C^{\rho \sigma}(P)$ in $\mathbf{G}_{K}^{(3)}$, Eq. (2.42)

The form for $H_{l}^{\rho \sigma}$ is obtained by using the formulae in Appendix B:

$$
\begin{aligned}
& H_{l}^{\rho \rho}=-C_{\rho-\rho}\left(N^{2} \Sigma_{A 3}^{-\rho \rho}-N^{2} \Sigma_{A 4}^{-\rho \rho} \not P+\Sigma_{A 1}^{-\rho \rho} \not \mathcal{X}-\Sigma_{A 2}^{-\rho \rho} \not P X^{\prime}\right) \\
& +\left(N^{2} \Sigma_{R 3}^{\rho-\rho}-N^{2} \Sigma_{R 4}^{\rho-\rho} \not P-\Sigma_{R 1}^{\rho-\rho} \not N+\Sigma_{R 2}^{\rho-\rho} \not P D X\right) C_{-\rho \rho}, \\
& H_{l}^{\rho-\rho}=\gamma_{5}\left[C_{\rho-\rho}\left(N^{2} \Sigma_{A 3}^{-\rho-\rho}-N^{2} \Sigma_{A 4}^{-\rho-\rho} \not P+\Sigma_{A 1}^{-\rho-\rho} \not \mathcal{X}-\Sigma_{A 2}^{-\rho-\rho} \not P X_{X}\right)\right. \\
& \left.+\left(N^{2} \Sigma_{R 3}^{\rho \rho}-N^{2} \Sigma_{R 4}^{\rho \rho} \not P-\Sigma_{R 1}^{\rho \rho} \not \mathcal{X}+\Sigma_{R 2}^{\rho \rho} \not P D X\right) C_{\rho-\rho}\right] .
\end{aligned}
$$


The form for $\gamma_{5} \lambda^{\top} \mathbf{C}^{\rho \sigma}(P)$ is given by Eq. (2.51) with

$$
\begin{aligned}
\Sigma_{A 1}^{\rho \sigma} \rightarrow \delta^{\rho \sigma}, \quad & \Sigma_{A j}^{\rho \sigma} \rightarrow 0 \quad(j=2-4) \\
\Sigma_{R j}^{\rho \sigma} \rightarrow 0 & (j=1-4)
\end{aligned}
$$

The form for $\hat{G}^{\rho-\rho}$

Having obtained the expression for $\hat{G}^{\rho \rho}$, we can get the expression for $\hat{G}^{\rho-\rho}$ from Eqs. Eq. (2.36), (2.39) - (2.42), by repeatedly using the formulae in Appendix B.

\section{GLUON PROPAGATOR}

\section{A. Preliminary}

We adopt a Coulomb gauge. The result for a covariant gauge is summarized in Appendix D.

As an orthogonal basis in Minkowski space, we choose

$$
\begin{array}{ll}
\tilde{P}^{\mu} \equiv P_{\mu}-p_{0} n^{\mu}=(0, \vec{p}), & \tilde{\zeta}^{\mu}=\left(0, \vec{\zeta}-(\vec{\zeta} \cdot \vec{p}) \vec{p} / \vec{p}^{2}\right), \\
n^{\mu}=(1, \overrightarrow{0}), & E_{\perp}^{\mu}=\epsilon^{\mu \nu \rho \sigma} \tilde{P}_{\nu} \tilde{\zeta}_{\rho} n_{\sigma} .
\end{array}
$$

These vectors are orthogonal with each other and their norms are

$$
\begin{array}{ll}
\tilde{P}^{2}=-\vec{p}^{2}, & \tilde{\zeta}^{2}=-1+(\vec{\zeta} \cdot \vec{p})^{2} / \vec{p}^{2}, \\
n^{2}=1, & E_{\perp}^{2}=\vec{p}^{2} \tilde{\zeta}^{2} .
\end{array}
$$

Incidentally, $\epsilon^{\mu \nu \rho \sigma} \tilde{P}_{\rho} \tilde{\zeta}_{\sigma}, \epsilon^{\mu \nu \rho \sigma} \tilde{P}_{\rho} n_{\sigma}$, and $\epsilon^{\mu \nu \rho \sigma} \tilde{\zeta}_{\rho} n_{\sigma}$ are not independent but are constructed out of the above four vectors, e.g., $\epsilon^{\mu \nu \rho \sigma} \tilde{P}_{\rho} \tilde{\zeta}_{\sigma}=\left(E_{\perp}^{\mu} n^{\nu}-n^{\mu} E_{\perp}^{\nu}\right)$, etc.

We define the projection operators,

$$
\begin{aligned}
& \mathcal{P}_{T}^{\mu \nu}(P)=g^{\mu \nu}-\frac{n^{\mu} n^{\nu}}{n^{2}}-\frac{\tilde{P}^{\mu} \tilde{P}^{\nu}}{\tilde{P}^{2}}, \\
& \mathcal{P}_{L}^{\mu \nu}(P)=\frac{n^{\mu} n^{\nu}}{n^{2}}, \\
& \mathcal{P}_{G}^{\mu \nu}(P)=\frac{\tilde{P}^{\mu} \tilde{P}^{\nu}}{\tilde{P}^{2}} .
\end{aligned}
$$


Although, $n^{2}=1$, we have written $n^{2}$ explicitly for later convenience. In the above definitions, ' $T$ ', ' $L$ ', and ' $G$ ' stand, in respective order, for transverse, longitudinal, and gauge fixing. (Following tradition, we call $n^{\mu} n^{\nu} / n^{2}$ in Eq. (3.3) the "longitudinal projection operator".) From Eqs. (3.1) - (3.4), one can show that

$$
\begin{aligned}
\tilde{P}_{\mu} \mathcal{P}_{U}^{\mu \nu} & =\mathcal{P}_{U}^{\nu \mu} \tilde{P}_{\mu}=\delta_{U G} \tilde{P}^{\nu}, \\
n_{\mu} \mathcal{P}_{U}^{\mu \nu} & =\mathcal{P}_{U}^{\nu \mu} n_{\mu}=\delta_{U L} n^{\nu}, \\
\tilde{\zeta}_{\mu} \mathcal{P}_{U}^{\mu \nu} & =\mathcal{P}_{U}^{\nu \mu} \tilde{\zeta}_{\mu}=\delta_{U T} \tilde{\zeta}^{\nu}, \\
\left(E_{\perp}\right)_{\mu} \mathcal{P}_{U}^{\mu \nu} & =\mathcal{P}_{U}^{\nu \mu}\left(E_{\perp}\right)_{\mu}=\delta_{U T} E_{\perp}^{\nu}
\end{aligned}
$$

Let $\mathbf{A}$ be a generic second-rank tensor in Minkowski space, whose $(\mu \nu)$-component is $(\mathbf{A})^{\mu \nu}=A^{\mu \nu} \cdot A^{\mu \nu}$ is decomposed as

$$
\begin{aligned}
A^{\mu \nu}(P)= & \sum_{U, V=T, L, G} \mathcal{P}_{U}^{\mu \rho}\left(A_{U V}\right)_{\rho \sigma} \mathcal{P}_{V}^{\sigma \nu} \\
\equiv & \sum_{U, V=T, L, G}\left(\mathcal{P}_{U} \cdot A_{U V} \cdot \mathcal{P}_{V}\right)^{\mu \nu} \\
A_{T T}^{\mu \nu}= & A_{1}^{T T} \mathcal{P}_{T}^{\mu \nu}+A_{2}^{T T} \tilde{\zeta}^{\mu} \tilde{\zeta}^{\nu}-A_{3}^{T T^{\prime}} \tilde{\zeta}^{\mu} E_{\perp}^{\nu} \\
& +A_{3}^{T^{\prime} T} E_{\perp}^{\mu} \tilde{\zeta}^{\nu} \\
A_{L L}^{\mu \nu}= & A_{1}^{L L} \mathcal{P}_{L}^{\mu \nu} \\
A_{G G}^{\mu \nu}= & A_{1}^{G G} \mathcal{P}_{G}^{\mu \nu}, \\
A_{T L}^{\mu \nu}= & A_{1}^{T L} \tilde{\zeta}^{\mu} n^{\nu}+A_{2}^{T L} E_{\perp}^{\mu} n^{\nu} \\
A_{L T}^{\mu \nu}= & A_{1}^{L T} n^{\mu} \tilde{\zeta}^{\nu}-A_{2}^{L T} n^{\mu} E_{\perp}^{\nu} \\
A_{T G}^{\mu \nu}= & A_{1}^{T G} E_{\perp}^{\mu} \tilde{P}^{\nu}+A_{2}^{T G} \tilde{\zeta}^{\mu} \tilde{P}^{\nu} \\
A_{G T}^{\mu \nu}= & A_{1}^{G T} \tilde{P}^{\mu} E_{\perp}^{\nu}-A_{2}^{G T} \tilde{P}^{\mu} \tilde{\zeta}^{\nu} \\
A_{L G}^{\mu \nu}= & A_{1}^{L G} n^{\mu} \tilde{P}^{\nu}, \\
A_{G L}^{\mu \nu}= & -A_{1}^{G L} \tilde{P}^{\mu} n^{\nu} .
\end{aligned}
$$

From Eq. (3.5), follows $\left(\mathcal{P}_{U} \cdot A_{U V} \cdot \mathcal{P}_{V}\right)^{\mu \nu}=A_{U V}^{\mu \nu}(U, V=T, L, G)$. We call Eqs. (3.6) and (3.7) the standard form $(\mathrm{SF})$ and refer $A_{U V}^{\mu \nu}(U, V=T, L, G)$ or $A_{j}^{U V}\left(U, V=T, T^{\prime}, L, G\right)$ 
to as an SF-element of $A^{\mu \nu}$. It is to be understood that the (bare and self-energy-part resummed) propagators and the self-energy part, which appear in the following, are to be written in the SF.

\section{B. Bare propagators}

\section{Bare gluon propagator}

First of all, we note that the bare propagator matrix $\hat{\mathbf{D}}(P)$ and the self-energy-part resummed propagator matrix $\hat{\mathbf{G}}(P)$ enjoy the "symmetry" property,

$$
\begin{aligned}
\left(\hat{D}^{\mu \nu}(P)\right)^{*} & =-\hat{\tau}_{1}^{\mathrm{t}} \hat{D}^{\nu \mu}(P) \hat{\tau}_{1}, \\
\left(\hat{G}^{\mu \nu}(P)\right)^{*} & =-\hat{\tau}_{1}^{\mathrm{t}} \hat{G}^{\nu \mu}(P) \hat{\tau}_{1}, \\
\hat{D}^{\mu \nu}(P) & ={ }^{\mathrm{t}} \hat{D}^{\nu \mu}(-P), \\
\hat{G}^{\mu \nu}(P) & ={ }^{\mathrm{t}} \hat{G}^{\nu \mu}(-P) .
\end{aligned}
$$

The first two equations results from the hermiticity of the density matrix.

$\hat{D}^{\mu \nu}(P)$ is an inverse of

$$
\left(\hat{D}^{-1}(P)\right)^{\mu \nu}=-\left[P^{2} g^{\mu \nu}-P^{\mu} P^{\nu}+\frac{1}{\lambda} \tilde{P}^{\mu} \tilde{P}^{\nu}\right] \hat{\tau}_{3}
$$

with $\lambda$ a gauge parameter. A general solution to $\left(\mathbf{D}^{-1} \mathbf{D}\right)^{\mu \nu}=g^{\mu \nu}$ is written as

$$
\begin{aligned}
\hat{\mathbf{D}}= & \hat{\mathbf{D}}^{(0)}+\mathbf{D}_{K} \hat{M}_{+}, \\
\hat{\mathbf{D}}^{(0)}= & \hat{\mathbf{D}}_{R A}+\tilde{f}\left(\mathbf{D}_{R}-\mathbf{D}_{A}\right) \hat{M}_{+}, \\
\hat{\mathbf{D}}_{R A}= & \left(\begin{array}{cc}
\mathbf{D}_{R} & 0 \\
\mathbf{D}_{R}-\mathbf{D}_{A} & -\mathbf{D}_{A}
\end{array}\right), \\
D_{K}^{\mu \nu}= & \left(D_{K}\right)_{T T}^{\mu \nu}=-\tilde{C}^{\mu \nu}\left(\Delta_{R}(P)-\Delta_{A}(P)\right), \\
\tilde{C}^{\mu \nu}= & C_{2}^{T T}(P) \tilde{\zeta}^{\mu} \tilde{\zeta}^{\nu}-C_{3}^{T T^{\prime}}(P) \tilde{\zeta}^{\mu} E_{\perp}^{\nu} \\
& +C_{3}^{T^{\prime} T}(P) E_{\perp}^{\mu} \tilde{\zeta}^{\nu},
\end{aligned}
$$


where

$$
\begin{array}{r}
\tilde{f}(P)=\theta\left(p_{0}\right) N\left(\left|p_{0}\right|, \vec{p}\right)-\theta\left(-p_{0}\right)\left[1+N\left(\left|p_{0}\right|,-\vec{p}\right)\right] \\
D_{R}^{\mu \nu}=\left(D_{A}^{\mu \nu}\right)^{*}=-\Delta_{R} \mathcal{P}_{T}^{\mu \nu}-\frac{1}{\tilde{P}^{2}}\left[1+\lambda \frac{p_{0}^{2}}{\tilde{P}^{2}}\right] \mathcal{P}_{L}^{\mu \nu} \\
\quad-\frac{\lambda}{\tilde{P}^{2}} \mathcal{P}_{G}^{\mu \nu}-\lambda \frac{p_{0}}{\tilde{P}^{4}}\left(\tilde{P}^{\mu} n^{\nu}+n^{\mu} \tilde{P}^{\nu}\right) .
\end{array}
$$

Here $N$ is the number density of the transverse gluon and $\Delta_{R(A)}$ is as in Eq. (2.11). From Eqs. (3.8), (3.10), and (3.13), we have

$$
\left(C_{2}^{T T}\right)^{*}=C_{2}^{T T}, \quad\left(C_{3}^{T T^{\prime}}\right)^{*}=-C_{3}^{T^{\prime} T}
$$

Note that, for the quasiuniform systems near equilibrium, $C_{2}^{T T}, C_{3}^{T T^{\prime}}$, and $C_{3}^{T^{\prime} T}$ are small when compared to $\tilde{f}$.

\section{Bare ghost propagator}

A bare Fadeev-Popov (FP) ghost propagator $\hat{\tilde{D}}$ is

$$
\hat{\tilde{D}}=\frac{1}{\tilde{P}^{2}} \hat{\tau}_{3}
$$

\section{Dyson equation}

\section{Gluon sector}

The self-energy-part $(\hat{\mathbf{\Pi}})$ resummed propagator $\hat{\mathbf{G}}$ obeys

$$
\hat{\mathbf{G}}(P)=\hat{\mathbf{D}}(P)-\hat{\mathbf{D}}(P) \hat{\mathbf{\Pi}}(P) \hat{\mathbf{G}}(P) .
$$

From Eq. (3.8), we obtain the symmetry relations, for the SF-elements of $\hat{G}^{\mu \nu}$, 


$$
\begin{aligned}
\left(\hat{G}_{j}^{U V}(P)\right)^{*} & =-\sigma_{j}^{U V} \hat{\tau}_{1}^{\mathrm{t}} \hat{G}_{j}^{V U}(P) \hat{\tau}_{1}, \\
\hat{G}_{j}^{U V}(P) & ={ }^{\mathrm{t}} \hat{G}_{j}^{V U}(-P) \quad\left(U, V=T, T^{\prime}, L, G\right),
\end{aligned}
$$

where $\sigma_{j}^{U V}=\sigma_{j}^{V U}$ with $\sigma_{j}^{U U}=+(U=T, L, G)$ and

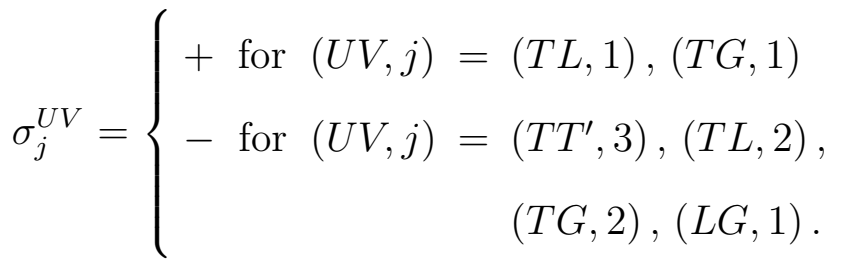

Similar relations hold for $\left(\hat{\Pi}_{j}\right)_{U V}$ 's.

Components of $\hat{\boldsymbol{\Pi}}$ follow the same relation as (2.19) and then $\hat{\boldsymbol{\Pi}}$ is written as

$$
\begin{aligned}
\hat{\boldsymbol{\Pi}} & =\hat{\boldsymbol{\Pi}}^{(0)}-\boldsymbol{\Pi}_{K} \hat{M}_{-}, \\
\hat{\boldsymbol{\Pi}}^{(0)} & =\left(\begin{array}{cc}
\boldsymbol{\Pi}_{R} & 0 \\
-\boldsymbol{\Pi}_{R}+\boldsymbol{\Pi}_{A} & -\boldsymbol{\Pi}_{A}
\end{array}\right)+\tilde{f}\left(\boldsymbol{\Pi}_{R}-\boldsymbol{\Pi}_{A}\right) \hat{M}_{-}, \\
\boldsymbol{\Pi}_{R} & =\boldsymbol{\Pi}_{11}+\boldsymbol{\Pi}_{12} \\
\boldsymbol{\Pi}_{A} & =\boldsymbol{\Pi}_{11}+\boldsymbol{\Pi}_{21}, \\
\boldsymbol{\Pi}_{K} & =(1+\tilde{f}) \boldsymbol{\Pi}_{12}-\tilde{f} \boldsymbol{\Pi}_{21} .
\end{aligned}
$$

From Eq. (3.19) with $\hat{\Pi}_{j}$ for $\hat{G}_{j}$, we obtain the symmetry relations $\left(U, V,=T, T^{\prime}, L, G\right)$,

$$
\begin{aligned}
\left(\Pi_{A j}^{U V}(P)\right)^{*}= & \sigma_{j}^{U V} \Pi_{R j}^{V U}(P) \\
\left(\Pi_{K j}^{U V}(P)\right)^{*}= & -\sigma_{j}^{U V} \Pi_{K j}^{V U}(P), \\
\Pi_{R j}^{U V}(P)= & \Pi_{A j}^{V U}(-P)=\sigma_{j}^{U V}\left(\Pi_{R j}^{U V}(-P)\right)^{*}, \\
\Pi_{K j}^{U V}(P)= & \Pi_{K j}^{V U}(-P) \\
& -\epsilon\left(p_{0}\right)\left[N\left(\left|p_{0}\right|, \vec{p}\right)-N\left(\left|p_{0}\right|,-\vec{p}\right)\right] \\
& \times\left(\Pi_{R j}^{V U}(-P)-\Pi_{A j}^{V U}(-P)\right) .
\end{aligned}
$$

Components of $\mathbf{G}$ follow the same relation as (2.25) and then $\hat{\mathbf{G}}$ is written as 


$$
\begin{aligned}
\hat{\mathbf{G}} & =\hat{\mathbf{G}}^{(0)}+\mathbf{G}_{K} \hat{M}_{+}, \\
\hat{\mathbf{G}}^{(0)} & =\left(\begin{array}{cc}
\mathbf{G}_{R} & 0 \\
\mathbf{G}_{R}-\mathbf{G}_{A} & -\mathbf{G}_{A}
\end{array}\right)+\tilde{f}\left(\mathbf{G}_{R}-\mathbf{G}_{A}\right) \hat{M}_{+}, \\
\mathbf{G}_{R} & =\mathbf{G}_{11}-\mathbf{G}_{12}, \\
\mathbf{G}_{A} & =\mathbf{G}_{11}-\mathbf{G}_{21}, \\
\mathbf{G}_{K} & =(1+\tilde{f}) \mathbf{G}_{12}-\tilde{f} \mathbf{G}_{21} .
\end{aligned}
$$

For equilibrium systems, $\boldsymbol{\Pi}_{K}=\mathbf{G}_{K}=0$. From Eq. (3.19), follows the symmetry relations $\left(U, V,=T, T^{\prime}, L, G\right)$,

$$
\begin{aligned}
\left(G_{A j}^{U V}(P)\right)^{*}= & \sigma_{j}^{U V} G_{R j}^{V U}(P), \\
\left(G_{K j}^{U V}(P)\right)^{*}= & -\sigma_{j}^{U V} G_{K j}^{V U}(P), \\
G_{R j}^{U V}(P)= & G_{A j}^{V U}(-P)=\sigma_{j}^{U V}\left(G_{R j}^{U V}(-P)\right)^{*}, \\
G_{K j}^{U V}(P)= & G_{K j}^{V U}(-P) \\
& +\epsilon\left(p_{0}\right)\left[N\left(\left|p_{0}\right|, \vec{p}\right)-N\left(\left|p_{0}\right|,-\vec{p}\right)\right] \\
& \times\left(G_{R j}^{V U}(-P)-G_{A j}^{V U}(-P)\right) .
\end{aligned}
$$

Substitution of Eqs. (3.10), (3.20), and (3.27) into Eq. (3.18) yields

$$
\begin{aligned}
\hat{\mathbf{G}}^{(0)} & =\hat{\mathbf{D}}^{(0)}-\hat{\mathbf{D}}^{(0)} \hat{\boldsymbol{\Pi}} \hat{\mathbf{G}}^{(0)} \\
\mathbf{G}_{K} & =\mathbf{D}_{K}-\mathbf{D}_{R} \boldsymbol{\Pi}_{R} \mathbf{G}_{K}+\mathbf{D}_{R} \boldsymbol{\Pi}_{K} \mathbf{G}_{A}-\mathbf{D}_{K} \boldsymbol{\Pi}_{A} \mathbf{G}_{A}
\end{aligned}
$$

Eq. (3.36) is formally solved to give

$$
\mathbf{G}_{R(A)}=\left[\mathbf{D}^{-1}+\boldsymbol{\Pi}_{R(A)}\right]^{-1}
$$

For later convenience, we rewrite Eq. (3.38) as (cf. Eq. (3.9)) 


$$
\begin{aligned}
\mathbf{G}_{R}= & {\left[\mathbf{D}_{0}^{-1}+\mathbf{\Pi}_{R}^{\prime}\right]^{-1} } \\
\left(D_{0}^{-1}\right)^{\mu \nu}= & -\tilde{P}^{2}\left[\mathcal{P}_{T}^{\mu \nu}+\mathcal{P}_{L}^{\mu \nu}+\frac{1}{\lambda} \mathcal{P}_{G}^{\mu \nu}\right] \\
\Pi_{R}^{\prime \mu \nu}= & \Pi_{R}^{\mu \nu}-p_{0}^{2}\left(\mathcal{P}_{T}^{\mu \nu}+\mathcal{P}_{G}^{\mu \nu}\right) \\
& +p_{0}\left(n^{\mu} \tilde{P}^{\nu}+\tilde{P}^{\mu} n^{\nu}\right)
\end{aligned}
$$

The $\mathrm{SF}$ for $\mathbf{G}_{R(A)}$ will be given in the next section.

As for $\mathbf{G}_{K}$, Eq. (3.37), through similar procedure as in the quark case (cf. Appendix A), we obtain

$$
\begin{aligned}
\mathbf{G}_{K} & =\mathbf{G}_{K}^{(1)}+\mathbf{G}_{K}^{(2)}+\mathbf{G}_{K}^{(3)} \\
\mathbf{G}_{K}^{(1)} & =\mathbf{G}_{R} \boldsymbol{\Pi}_{K} \mathbf{G}_{A}, \\
\mathbf{G}_{K}^{(2)} & =-\mathbf{G}_{R}\left[\tilde{\mathbf{C}} \boldsymbol{\Pi}_{A}-\boldsymbol{\Pi}_{R} \tilde{\mathbf{C}}\right] \mathbf{G}_{A} \\
& \equiv-\mathbf{G}_{R} \tilde{\mathbf{H}}_{l} \mathbf{G}_{A}, \\
\mathbf{G}_{K}^{(3)} & =\mathbf{G}_{R} \tilde{\mathbf{C}}-\tilde{\mathbf{C}} \mathbf{G}_{A},
\end{aligned}
$$

where $\tilde{\mathbf{C}}$ is as in Eq. (3.14). SF-elements of $\tilde{\mathbf{H}}_{l}$ is given in the next section. The SF elements of $\mathbf{G}_{K}$ is obtained by repeatedly using the formulae in Appendix C.

\section{Ghost sector}

The self-energy-part $(\hat{\tilde{\Pi}})$ resummed propagator $\hat{\tilde{G}}$ obeys

$$
\hat{\tilde{G}}(P)=\hat{\tilde{D}}(P)[1+\hat{\tilde{\Pi}}(P) \hat{\tilde{G}}(P)]=[1+\hat{\tilde{G}}(P) \hat{\tilde{\Pi}}(P)] \hat{\tilde{D}}
$$

Since, $\hat{\tilde{D}}$, Eq. (3.17), is a diagonal $(2 \times 2)$-matrix, $\hat{\tilde{\Pi}}$ is also diagonal. Then, from Eq. (3.43), $\hat{\tilde{G}}$ is diagonal also. Among the components of $\hat{\tilde{\Pi}}(\hat{\tilde{G}})$, there is the same relation as $(2.19)$ $((2.25))$. Then we have

$$
\hat{\tilde{\Pi}}=\tilde{\Pi} \hat{\tau}_{3}, \quad \hat{\tilde{G}}=\tilde{G} \hat{\tau}_{3}
$$

with $\tilde{\Pi}$ and $\tilde{G}$ real, and

$$
\tilde{G}(P)=\frac{1}{\tilde{P}^{2}-\tilde{\Pi}(P)}=-\frac{1}{\vec{p}^{2}+\tilde{\Pi}(P)} .
$$




\section{Self-energy-part resummed gluon propagator}

$$
\text { Form for }\left(G_{R(A)}\right)_{U V}
$$

Through a Slavnov-Taylor identity, $\left(\hat{G}_{1}\right)_{U G}(U=G, T, L)$ and $\left(\hat{G}_{2}\right)_{T G}$ (and then also $\left(\hat{G}_{1}\right)_{G U}$ and $\left(\hat{G}_{2}\right)_{G T}$ via Eq. (3.19)) are related to the self-energy-part resummed FP-ghost propagator $\hat{\tilde{G}}$ and the FP-ghost "pre self-energy part" ${ }^{1} \hat{\tilde{\Pi}}_{\mu}$.

The Slavnov-Taylor identity reads [7]:

$$
\begin{aligned}
\hat{G}_{\mu \nu} \tilde{P}^{\nu} & =\lambda\left[\hat{\tau}_{3} \hat{\tilde{\Pi}}_{\mu}-P_{\mu}\right] \hat{\tilde{G}} \\
& =\lambda\left[\hat{\tau}_{3}\left(\hat{\tilde{\Pi}}_{\mu}-p_{0} n_{\mu} \hat{\tau}_{3}\right)-\tilde{P}_{\mu}\right] \hat{\tilde{G}} .
\end{aligned}
$$

Here $\hat{\tilde{G}}(P)$ is as in Eq. (3.44) with Eq. (3.45). As in Eq. (3.44), $\hat{\tilde{\Pi}}_{\mu}$ is diagonal $(2 \times 2)$-matrix,

$$
\hat{\tilde{\Pi}}_{\mu}=\tilde{\Pi}_{\mu} \hat{\tau}_{3} \quad\left(\tilde{\Pi}_{\mu}^{*}=\tilde{\Pi}_{\mu}\right)
$$

Substitution of the SF for $\hat{G}^{\mu \nu}$ into Eq. (3.46) yields

$$
\begin{aligned}
G_{R 1}^{G G}(P) & =G_{A 1}^{G G}(P)=\lambda \frac{1}{\tilde{P}^{2}}\left[\tilde{\Pi}(P)-\tilde{P}^{2}\right] \tilde{G}(P) \\
& =-\lambda \frac{1}{\tilde{P}^{2}}, \\
G_{R 1}^{T G}(P) & =G_{A 1}^{T G}(P)=\lambda \frac{1}{\tilde{P}^{2} E_{\perp}^{2}}\left(E_{\perp}^{\mu} \tilde{\Pi}_{\mu}(P)\right) \tilde{G}(P), \\
G_{R 2}^{T G}(P) & =G_{A 2}^{T G}(P)=\lambda \frac{1}{\tilde{P}^{2} \tilde{\zeta}^{2}}\left(\tilde{\zeta}^{\mu} \tilde{\Pi}_{\mu}(P)\right) \tilde{G}(P), \\
G_{R 1}^{L G}(P) & =G_{A 1}^{L G}(P)=\lambda \frac{1}{\tilde{P}^{2} n^{2}}\left(n^{\mu} \tilde{\Pi}_{\mu}(P)-p_{0}\right) \tilde{G}(P), \\
G_{K 1}^{G G} & =G_{K 1}^{T G}=G_{K 2}^{T G}=G_{K 1}^{L G}=0 .
\end{aligned}
$$

\footnotetext{
${ }^{1} \hat{\tilde{\Pi}}_{\mu}$ is evaluated by replacing the vertex factor $g C_{a b c} \tilde{P}^{\mu}$ at the "end vertex" with $g C_{a b c}$. Here the "end vertex" is the vertex from which the outgoing ghost comes out of the diagram. Then, the ghost self-energy part $\hat{\tilde{\Pi}}$ is related to $\hat{\tilde{\Pi}}_{\mu}$ through $\hat{\tilde{\Pi}}=\tilde{P}^{\mu} \hat{\tilde{\Pi}}_{\mu}$.
} 
All the above quantities are real. In deriving Eq. (3.48), Eq. (3.45) has been used. Substituting Eqs. (3.48) - (3.52) into Eq. (3.35), we obtain $G_{K j}^{G T}=G_{K 1}^{G L}=0$. From the above formulae, we see that $\hat{G}_{U G}^{\mu \nu}(U=T, L, G)$ and $\hat{G}_{G U}^{\mu \nu}(U=T, L)$ vanish in the strict Coulomb gauge $(\lambda=0)$.

We are now in a position to obtain $\left(\mathbf{G}_{R}\right)_{U V}(U \neq G, V \neq G)$ from Eq. (3.39). We divide $\left(\mathbf{G}_{R}\right)_{U V}$ into two pieces, $\left(\mathbf{G}_{R}\right)_{U V}=\left(\mathbf{G}_{R}^{(\lambda=0)}\right)_{U V}+\left(\mathbf{G}_{R}^{(\lambda)}\right)_{U V}$, the latter of which vanishes in the strict Coulomb gauge $(\lambda=0)$.

Straightforward manipulation of Eq. (3.39) using the formulae in Appendix C yields, for the SF-elements of $\mathbf{G}^{(\lambda=0)}\left(\equiv \mathbf{G}_{R}^{(\lambda=0)}\right)\left(\Pi_{j}^{U V} \equiv \Pi_{R j}^{U V}\right)$,

$$
\begin{aligned}
\mathcal{D} G_{1}^{(\lambda=0) T T} & =-\left(\tilde{P}^{2}-\Pi_{1}^{\prime T T}-\tilde{\zeta}^{2} \Pi_{2}^{T T}\right)\left(\tilde{P}^{2}-\Pi_{1}^{L L}\right)+\tilde{\zeta}^{2} n^{2} \Pi_{1}^{T L} \Pi_{1}^{L T}, \\
\mathcal{D} G_{2}^{(\lambda=0) T T} & =-\left(\tilde{P}^{2}-\Pi_{1}^{L L}\right) \Pi_{2}^{T T}+\tilde{P}^{2} n^{4} \Pi_{2}^{T L} \Pi_{2}^{L T}+n^{2} \Pi_{1}^{T L} \Pi_{1}^{L T}, \\
\mathcal{D} G_{3}^{(\lambda=0) T^{\prime} T} & =-\left(\tilde{P}^{2}-\Pi_{1}^{L L}\right) \Pi_{3}^{T^{\prime} T}-n^{2} \Pi_{1}^{L T} \Pi_{2}^{T L}, \\
\mathcal{D} G_{1}^{(\lambda=0) L L} & =-\left(\tilde{P}^{2}-\Pi_{1}^{\prime T T}-\tilde{\zeta}^{2} \Pi_{2}^{T T}\right)\left(\tilde{P}^{2}-\Pi_{1}^{\prime T T}\right)+\tilde{P}^{2} \tilde{\zeta}^{4} n^{2} \Pi_{3}^{T T^{\prime}} \Pi_{3}^{T^{\prime} T}, \\
\mathcal{D} G_{2}^{(\lambda=0) T L} & =-\left(\tilde{P}^{2}-\Pi_{1}^{\prime T T}-\tilde{\zeta}^{2} \Pi_{2}^{T T}\right) \Pi_{2}^{T L}-\tilde{\zeta}^{2} \Pi_{1}^{T L} \Pi_{3}^{T^{\prime} T}, \\
\mathcal{D} G_{1}^{(\lambda=0) L T} & =-\left(\tilde{P}^{2}-\Pi_{1}^{\prime T T}\right) \Pi_{1}^{L T}-\tilde{P}^{2} \tilde{\zeta}^{2} n^{2} \Pi_{2}^{L T} \Pi_{3}^{T^{\prime} T},
\end{aligned}
$$

where

$$
\begin{aligned}
\mathcal{D}= & {\left[\left(\tilde{P}^{2}-\Pi_{1}^{\prime T T}\right)\left(\tilde{P}^{2}-\Pi_{1}^{L L}\right)-\tilde{P}^{2} \tilde{\zeta}^{2} n^{4} \Pi_{2}^{L T} \Pi_{2}^{T L}\right]\left(\tilde{P}^{2}-\Pi_{1}^{\prime T T}-\tilde{\zeta}^{2} \Pi_{2}^{T T}\right) } \\
& -\tilde{\zeta}^{2} n^{2} \Pi_{1}^{L T}\left[\left(\tilde{P}^{2}-\Pi_{1}^{\prime T T}\right) \Pi_{1}^{T L}+\tilde{P}^{2} \tilde{\zeta}^{2} n^{2} \Pi_{3}^{T T^{\prime}} \Pi_{2}^{T L}\right] \\
& +\vec{p}^{2} \tilde{\zeta}^{4} \Pi_{3}^{T^{\prime} T}\left[\left(\tilde{P}^{2}-\Pi_{1}^{L L}\right) \Pi_{3}^{T T^{\prime}}+n^{2} \Pi_{1}^{T L} \Pi_{2}^{L T}\right] .
\end{aligned}
$$

Here, we note that, from Eq. (3.41), $\tilde{P}^{2}-\Pi_{1}^{\prime T T}=P^{2}-\Pi_{1}^{T T}$ holds. The SF-elements of the gauge-parameter dependent part $\mathbf{G}^{(\lambda)} \equiv \mathbf{G}_{R}^{(\lambda)}$ reads

$$
\begin{aligned}
& \mathcal{D} G_{1}^{(\lambda) T T}=-\tilde{P}^{4} \tilde{\zeta}^{2} n^{2}\left[\left(\tilde{P}^{2}-\Pi_{1}^{\prime T T}-\tilde{\zeta}^{2} \Pi_{2}^{T T}\right)\left\{\left(\tilde{P}^{2}-\Pi_{1}^{L L}\right) \Pi_{1}^{G T}+n^{2} \Pi_{1}^{\prime G L} \Pi_{2}^{L T}\right\}\right. \\
& +\tilde{\zeta}^{2} \Pi_{2}^{G T}\left\{\left(\tilde{P}^{2}-\Pi_{1}^{L L}\right) \Pi_{3}^{T T^{\prime}}+n^{2} \Pi_{1}^{T L} \Pi_{2}^{L T}\right\} \\
& \left.-\tilde{\zeta}^{2} n^{2} \Pi_{1}^{L T}\left\{\Pi_{1}^{G T} \Pi_{1}^{T L}-\Pi_{1}^{\prime} G L \Pi_{3}^{T T^{\prime}}\right\}\right] G_{1}^{T G},
\end{aligned}
$$




$$
\begin{aligned}
& \mathcal{D} G_{2}^{(\lambda) T T}=-\frac{\tilde{P}^{2}}{\tilde{\zeta}^{2} \Pi_{3}^{T^{\prime} T}}\left[\left\{\left(\tilde{P}^{2}-\Pi_{1}^{L L}\right) \Pi_{2}^{G T}+n^{2} \Pi_{1}^{\prime G L} \Pi_{1}^{L T}\right\}\left(\tilde{P}^{2}-\Pi_{1}^{\prime T T}\right)^{2}\right. \\
& +\tilde{P}^{2} \tilde{\zeta}^{2} n^{4} \Pi_{2}^{T L}\left(\tilde{P}^{2}-\Pi_{1}^{\prime T T}\right)\left\{\Pi_{1}^{L T} \Pi_{1}^{G T}-\Pi_{2}^{L T} \Pi_{2}^{G T}\right\} \\
& -\tilde{P}^{2} \tilde{\zeta}^{4} n^{2} \Pi_{3}^{T^{\prime} T}\left\{\left(\tilde{P}^{2}-\Pi_{1}^{L L}\right)\left(\Pi_{2}^{G T} \Pi_{3}^{T T^{\prime}}-\Pi_{1}^{G T} \Pi_{2}^{T T}\right)\right. \\
& \left.\left.+n^{2} \Pi_{1}^{\prime G L}\left(\Pi_{1}^{L T} \Pi_{3}^{T T^{\prime}}-\Pi_{2}^{T T} \Pi_{2}^{L T}\right)+n^{2} \Pi_{1}^{T L}\left(\Pi_{2}^{G T} \Pi_{2}^{L T}-\Pi_{1}^{G T} \Pi_{1}^{L T}\right)\right\}\right] G_{1}^{T G} \\
& -\frac{n^{2}}{\tilde{\zeta}^{2}} \frac{\Pi_{2}^{T L} G_{1}^{L G}}{\prod_{3}^{T^{\prime} T} G_{1}^{T G}} G_{3}^{(\lambda) T^{\prime} T} \mathcal{D}+\frac{\tilde{P}^{2}}{\tilde{\zeta}^{4}} \frac{\Pi_{1}^{T G}}{\prod_{3}^{T^{\prime} T}} G_{2}^{G T} \mathcal{D}, \\
& \mathcal{D} G_{3}^{(\lambda) T^{\prime} T}=\tilde{P}^{2}\left[-\tilde{P}^{2} \tilde{\zeta}^{2} n^{2} \Pi_{1}^{G T}\left\{\left(\tilde{P}^{2}-\Pi_{1}^{L L}\right) \Pi_{3}^{T^{\prime} T}+n^{2} \Pi_{1}^{L T} \Pi_{2}^{T L}\right\}\right. \\
& -\Pi_{2}^{G T}\left\{\left(\tilde{P}^{2}-\Pi_{1}^{T T}\right)\left(\tilde{P}^{2}-\Pi_{1}^{L L}\right)-\tilde{P}^{2} \tilde{\zeta}^{2} n^{4} \Pi_{2}^{T L} \Pi_{2}^{L T}\right\} \\
& \left.-n^{2} \Pi_{1}^{\prime G L}\left\{\left(\tilde{P}^{2}-\Pi_{1}^{\prime T T}\right) \Pi_{1}^{L T}+\tilde{P}^{2} \tilde{\zeta}^{2} n^{2} \Pi_{2}^{L T} \Pi_{3}^{T^{\prime} T}\right\}\right] G_{1}^{T G}, \\
& \mathcal{D} G_{1}^{(\lambda) L L}=\vec{p}^{2}\left[\left(\tilde{P}^{2}-\Pi_{1}^{\prime T T}-\tilde{\zeta}^{2} \Pi_{2}^{T T}\right)\left\{\left(\tilde{P}^{2}-\Pi_{1}^{\prime T T}\right) \Pi_{1}^{\prime G L}+\tilde{P}^{2} \tilde{\zeta}^{2} n^{2} \Pi_{1}^{G T} \Pi_{2}^{T L}\right\}\right. \\
& +\tilde{\zeta}^{2} \Pi_{2}^{G T}\left\{\left(\tilde{P}^{2}-\Pi_{1}^{\prime T T}\right) \Pi_{1}^{T L}+\tilde{P}^{2} \tilde{\zeta}^{2} n^{2} \Pi_{2}^{T L} \Pi_{3}^{T T^{\prime}}\right\} \\
& \left.-\tilde{P}^{2} \tilde{\zeta}^{4} n^{2} \Pi_{3}^{T^{\prime} T}\left\{\Pi_{1}^{\prime G L} \Pi_{3}^{T T^{\prime}}-\Pi_{1}^{G T} \Pi_{1}^{T L}\right\}\right] G_{1}^{L G}, \\
& \mathcal{D} G_{2}^{(\lambda) T L}=\tilde{P}^{2}\left[-\Pi_{1}^{\prime} G L\left(\tilde{P}^{2}-\Pi_{1}^{\prime T T}\right)^{2}+\tilde{P}^{2} \tilde{\zeta}^{4} n^{2} \Pi_{1}^{G T}\left\{\Pi_{2}^{T T} \Pi_{2}^{T L}-\Pi_{1}^{T L} \Pi_{3}^{T^{\prime} T}\right\}\right. \\
& +\tilde{\zeta}^{2}\left(\tilde{P}^{2}-\Pi_{1}^{\prime T T}\right)\left\{-\tilde{P}^{2} n^{2} \Pi_{1}^{G T} \Pi_{2}^{T L}+\Pi_{2}^{T T} \Pi_{1}^{\prime G L}-\Pi_{2}^{G T} \Pi_{1}^{T L}\right\} \\
& \left.-\tilde{P}^{2} \tilde{\zeta}^{4} n^{2} \Pi_{3}^{T T^{\prime}}\left\{\Pi_{2}^{G T} \Pi_{2}^{T L}-\Pi_{1}^{\prime G L} \Pi_{3}^{T^{\prime} T}\right\}\right] G_{1}^{T G}, \\
& G_{1}^{(\lambda) L T}=G_{3}^{(\lambda) T^{\prime} T} \frac{G_{1}^{L G}}{G_{1}^{T G}}
\end{aligned}
$$

Here, $G_{1}^{T G}\left(\equiv G_{R 1}^{T G}\right)$ and $G_{1}^{L G}\left(\equiv G_{R 1}^{L G}\right)$ are as in Eqs. (3.49) and (3.51), respectively. $G_{2}^{G T}$ $\left(\equiv G_{R 2}^{G T}\right)$ is obtained from $G_{A 2}^{T G}$, Eq. (3.50), with the help of Eq. (3.32).

$\left(\mathbf{G}_{A}\right)_{U V}$ is obtained from th above formulae with the substitutions $\Pi_{j}^{U V}\left(\equiv \Pi_{R j}^{U V}\right) \rightarrow$ $\Pi_{A j}^{U V} . G_{R 3}^{T T^{\prime}}, G_{R 1}^{T L}$, and $G_{R 2}^{L T}$ are obtained, in respective order, from $G_{A 3}^{T^{\prime} T}, G_{A 1}^{L T}$, and $G_{A 2}^{T L}$ with the help of Eq. (3.32) or Eq. (3.34).

$$
\text { Form for }\left(\tilde{H}_{l}\right)_{U V} \text { in Eq. (3.42) }
$$

Straightforward computation using the formulae in Appendix C yields, for the SFelements of $\tilde{H}_{l}$, 


$$
\begin{aligned}
\left(\tilde{H}_{l}\right)_{1}^{T T}= & 2 i \tilde{\zeta}^{2} E_{\perp}^{2} \operatorname{Im}\left(C_{3}^{T T^{\prime}} \Pi_{R 3}^{T^{\prime} T}\right) \\
\left(\tilde{H}_{l}\right)_{2}^{T T}= & 2 i \operatorname{Im}\left\{-C_{2}^{T T}\left(\Pi_{R 1}^{T T}+\tilde{\zeta}^{2} \Pi_{R 2}^{T T}\right)\right. \\
& \left.+E_{\perp}^{2} C_{3}^{T^{\prime} T}\left(\Pi_{R 3}^{T T^{\prime}}+\Pi_{A 3}^{T T^{\prime}}\right)\right\}, \\
\left(\tilde{H}_{l}\right)_{3}^{T T^{\prime}}= & \tilde{\zeta}^{2} C_{2}^{T T} \Pi_{A 3}^{T T^{\prime}}-C_{3}^{T T^{\prime}}\left(2 i I m \Pi_{R 1}^{T T}+\tilde{\zeta}^{2} \Pi_{R 2}^{T T}\right), \\
\left(\tilde{H}_{l}\right)_{1}^{T L}= & \tilde{\zeta}^{2} C_{2}^{T T} \Pi_{A 1}^{T L}-E_{\perp}^{2} C_{3}^{T T^{\prime}} \Pi_{A 2}^{T L}, \\
\left(\tilde{H}_{l}\right)_{2}^{T L}= & \tilde{\zeta}^{2} C_{3}^{T^{\prime} T} \Pi_{A 1}^{T L} .
\end{aligned}
$$

$\left(\tilde{H}_{l}\right)_{3}^{T^{\prime} T},\left(\tilde{H}_{l}\right)_{1}^{L T}$, and $\left(\tilde{H}_{l}\right)_{2}^{L T}$ are obtained using Eqs. (3.32) and (3.35). Other SF-elements than the above ones vanish.

\section{GRADIENT PARTS OF THE PROPAGATORS AND THE GENERALIZED BOLTZMANN EQUATIONS}

Here, we deduce the gradient terms of the quark and gluon propagators, and derive generalized Boltzmann equations and their relatives. Procedure goes parallel to those in [8-10], and then we describe briefly.

\section{A. Quark sector}

A configuration-space counterpart of $F(P, X)$ is denoted by $\underline{F}(x, y)$ :

$$
\begin{aligned}
\underline{F}(x, y) & =\int \frac{d^{4} P}{(2 \pi)^{4}} e^{-i P \cdot(x-y)} F(P, X) \quad\left(X=\frac{x+y}{2}\right), \\
& \equiv(F(P, X))_{\mathrm{IWT}}(x, y) .
\end{aligned}
$$

If $F(P, X)$ is independent of $\mathrm{X}, \underline{F}(x, y)=\underline{F}(x-y) \equiv(F(P))_{\mathrm{IFT}}(x-y)$. Here "IWT" ("IFT") stands for an inverse Wigner (Fourier) transform.

\section{Preliminary}

Configuration-space counterparts of Eqs. (2.4) and (2.5) are, with obvious notation, 


$$
\begin{aligned}
\underline{A}(x, y)= & \sum_{\rho, \sigma= \pm}\left[\underline{\mathcal{P}}_{\rho} \cdot \underline{A}^{\rho \sigma} \cdot \underline{\mathcal{P}}_{\sigma}\right](x, y), \\
\underline{A}^{\rho \rho}= & \underline{A}_{1}^{\rho \rho}+\frac{1}{2}\left(\underline{A}_{2}^{\rho \rho} \cdot(i \not \partial)+(i \not \partial) \cdot \underline{A}_{2}^{\rho \rho}\right) \\
& +\frac{1}{2}\left(\underline{A}_{3}^{\rho \rho} \cdot \underline{\underline{X}}+\underline{\underline{X}} \cdot \underline{A}_{3}^{\rho \rho}\right) \\
& +\frac{1}{2}\left(\underline{A}_{4}^{\rho \rho} \cdot(i \not \partial) \cdot \underline{\underline{X}}+(i \not \partial) \cdot \underline{\underline{X}} \cdot \underline{A}_{4}^{\rho \rho}\right), \\
\underline{A}^{\rho-\rho}= & \gamma_{5}\left[\underline{A}_{1}^{\rho-\rho}+\frac{1}{2}\left(\underline{A}_{2}^{\rho-\rho} \cdot(i \not \partial)+(i \not \partial) \cdot \underline{A}_{2}^{\rho-\rho}\right)\right. \\
& +\frac{1}{2}\left(\underline{A}_{3}^{\rho-\rho} \cdot \underline{\underline{X}}+\underline{\underline{X}} \cdot \underline{A}_{3}^{\rho-\rho}\right) \\
& \left.+\frac{1}{2}\left(\underline{A}_{4}^{\rho-\rho} \cdot(i \not \partial) \cdot \underline{\underline{X}}+(i \not \partial) \cdot \underline{\underline{X}} \cdot \underline{A}_{4}^{\rho-\rho}\right)\right] .
\end{aligned}
$$

Here we have used the short-hand notation $\underline{F} \cdot \underline{G}$, which is a function whose " $(x, y)$ component" is

$$
[\underline{F} \cdot \underline{G}](x, y)=\int d^{4} z \underline{F}(x, z) \underline{G}(z, y) .
$$

For later use, we display the Wigner transform of $\underline{F}_{G}=\underline{F} \cdot \underline{G}$ :

$$
F_{G}(P, X)=F(P, X) G(P, X)-\frac{i}{2}\{F(P, X), G(P, X)\},
$$

which is valid to the gradient approximation. The "Poisson bracket" in Eq. (4.3) is defined as

$$
\{F, G\} \equiv \frac{\partial F}{\partial X^{\mu}} \frac{\partial G}{\partial P_{\mu}}-\frac{\partial F}{\partial P_{\mu}} \frac{\partial G}{\partial X^{\mu}}
$$

\section{Bare propagator and counter-Lagrangian}

We proceed as in [9]. We start from the expression for the free propagator $\underline{\hat{S}}(x, y)$ (cf. Eqs. (2.8) - (2.10)),

$$
\begin{aligned}
\underline{\hat{S}}(x, y) & =\sum_{\rho= \pm} \underline{\mathcal{P}}_{\rho} \cdot \underline{\hat{S}}_{\rho} \cdot \underline{\mathcal{P}}_{\rho}+\underline{S}_{K} \hat{M}_{+}, \\
\underline{\hat{S}}_{\rho} & =\left(\begin{array}{cc}
\underline{\hat{S}}_{R} & 0 \\
\underline{\hat{S}}_{R}-\underline{\hat{S}}_{A} & -\underline{\hat{S}}_{A}
\end{array}\right)
\end{aligned}
$$




$$
\begin{aligned}
& -\left(\underline{\hat{S}}_{R} \cdot \underline{f}_{\rho}-\underline{f}_{\rho} \cdot \underline{\hat{S}}_{A}\right) \hat{M}_{+}, \\
\underline{S}_{K}= & \sum_{\rho= \pm} \underline{\mathcal{P}}_{\rho} \cdot\left(\underline{S}_{R} \cdot \gamma_{5} \underline{\underline{X}} \cdot \underline{C}_{\rho-\rho}\right. \\
& \left.-\underline{C}_{\rho-\rho} \cdot \gamma_{5} \underline{\underline{X}} \cdot \underline{S}_{A}\right) \cdot \underline{\mathcal{P}}_{-\rho} .
\end{aligned}
$$

For the time being, $\underline{f}_{\rho}(x, y)$ and $\underline{C}_{\rho-\rho}(x, y)$ in the above equations are left to be arbitrary. Specification of them will be made in Subsubsec. 5. $\hat{S}$ in Eq. (2.8) is the leading part of the

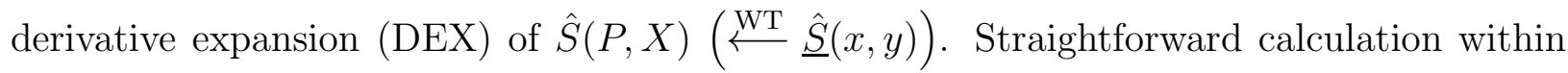
the gradient approximation yields

$$
\begin{aligned}
& \underline{\hat{S}}^{-1} \cdot \underline{\hat{S}}=\underline{\hat{S}} \cdot \underline{\hat{S}}^{-1}=1, \\
& \underline{\hat{S}}^{-1}(x, y)=\left(i \phi_{x}-m\right) \delta^{4}(x-y) \hat{\tau}_{3}-\underline{L}_{c}(x, y) \hat{M}_{-}, \\
& \underline{L}_{c}=\underline{L}_{c 1}+\underline{L}_{c 2}, \\
& L_{c 1}=i \sum_{\rho= \pm}\left(\not_{X} f_{\rho}\right) \mathcal{P}_{\rho} \\
& =i \sum_{\rho= \pm} \mathcal{P}_{\rho}\left[\frac{P \cdot \partial_{X}}{P^{2}} \not P+\frac{N \cdot \partial_{X}}{N^{2}} \not \mathcal{X}+\frac{\rho \epsilon\left(p_{0}\right)}{e_{\perp}^{2}}\left(e_{\perp} \cdot \partial_{X}\right) \not P X\right] f_{\rho} \mathcal{P}_{\rho}, \\
& L_{c 2}=i \sum_{\rho= \pm} \mathcal{P}_{\rho} \gamma_{5}\left[\frac{\partial X}{\partial P_{\alpha}} \frac{\partial C_{\rho-\rho}}{\partial X^{\alpha}}(\not P-m)+\left(\not \not_{X} C_{\rho-\rho}\right) \not X\right] \mathcal{P}_{-\rho} \\
& =i \sum_{\rho= \pm} \mathcal{P}_{\rho} \gamma_{5}\left[\left\{-\frac{\rho \epsilon\left(p_{0}\right)}{N^{2}} \not i e_{\perp}^{\mu} \frac{\partial N_{\mu}}{\partial P_{\alpha}}-\frac{\not P X}{2 N^{2}} \frac{\partial N^{2}}{\partial P_{\alpha}}\right.\right. \\
& \left.-m\left(-\frac{\not P}{P^{2}} N^{\alpha}+\frac{\not \mathcal{X}}{2 N^{2}} \frac{\partial N^{2}}{\partial P_{\alpha}}-\frac{\rho \epsilon\left(p_{0}\right)}{e_{\perp}^{2}} \not P \mathcal{X}_{\perp}^{\mu} \frac{\partial N^{\mu}}{\partial P_{\alpha}}\right)\right\} \frac{\partial C_{\rho-\rho}}{\partial X^{\alpha}} \\
& \left.+\left\{\frac{\rho \epsilon\left(p_{0}\right)}{P^{2}} \not P\left(e_{\perp} \cdot \partial_{X}\right)+\frac{\not P \not X}{P^{2}}\left(P \cdot \partial_{X}\right)\right\} C_{\rho-\rho}\right] \mathcal{P}_{-\rho} \text {. }
\end{aligned}
$$

Here $1 / P^{2} \equiv \mathbf{P} / P^{2}, 1 / N^{2} \equiv \mathbf{P} / N^{2}$, and $1 / e_{\perp}^{2}=\mathbf{P} / e_{\perp}^{2}$, with $\mathbf{P}$ denoting to take the principal part.

Eq. (4.5) tells us that the free action of the theory $[6,9]$ is

$$
\begin{gathered}
\mathcal{A}_{0}=\int d^{4} x d^{4} y \hat{\bar{\psi}}(x) \underline{\hat{S}}^{-1}(x, y) \hat{\psi}(y), \\
\hat{\bar{\psi}}=\left(\bar{\psi}_{1}, \bar{\psi}_{2}\right), \quad \hat{\psi}=\left(\begin{array}{l}
\psi_{1} \\
\psi_{2}
\end{array}\right) .
\end{gathered}
$$


Since the term with $\underline{L}_{c}(x, y)\left(\in \underline{\hat{S}}^{-1}\right)$ in $\mathcal{A}_{0}$ is absent in the original action, we should introduce a counteraction to compensate it:

$$
\mathcal{A}_{c}=\int d^{4} x d^{4} y \hat{\bar{\psi}}(x) \underline{L}_{c}(x, y) \hat{M}_{-} \hat{\psi}(y)
$$

which yields a (two-point) vertex factor

$$
i \underline{L}_{c}(x, y) \hat{M}_{-} \stackrel{\mathrm{WT}}{\longrightarrow} i\left(L_{c 1}(P, X)+L_{c 2}(P, X)\right) \hat{M}_{-} .
$$

Here "WT" indicates to take Wigner transformation.

\section{Dyson equation}

Let us start with considering a "product" of $\underline{A}$ and $\underline{B}$ of the type (4.1) (cf. Eq. (4.2)),

$$
\begin{aligned}
\underline{C}(x, y) & =[\underline{A} \cdot \underline{B}](x, y) \\
& =\left[\sum_{\rho, \xi, \sigma= \pm} \underline{\mathcal{P}}_{\rho} \cdot \underline{A}^{\rho \xi} \cdot \underline{\mathcal{P}}_{\xi} \cdot \underline{B}^{\xi \sigma} \cdot \underline{\mathcal{P}}_{\sigma}\right](x, y) .
\end{aligned}
$$

Using Eq. (4.3), we obtain, for the Wigner transform of $\underline{C}$ to the gradient approximation,

$$
\begin{aligned}
C(P, X)= & \sum_{\rho, \xi, \sigma= \pm}\left[\mathcal{P}_{\rho} A^{\rho \xi}(P, X) B^{\xi \sigma}(P, X) \mathcal{P}_{\sigma}\right. \\
& +\frac{i}{2} \mathcal{P}_{\rho}\left\{A^{\rho \xi} \frac{\partial \mathcal{P}_{\xi}}{\partial P_{\mu}} \frac{\partial B^{\xi \sigma}}{\partial X^{\mu}}\right. \\
& \left.\left.-\frac{\partial A^{\rho \xi}}{\partial X^{\mu}} \frac{\partial \mathcal{P}_{\xi}}{\partial P_{\mu}} B^{\xi \sigma}\right\} \mathcal{P}_{\sigma}+\ldots\right]
\end{aligned}
$$

where "..." stands for other pieces of the gradient terms than the second term. Thanks to the relation,

$$
\mathcal{P}_{ \pm \rho} \frac{\partial \mathcal{P}_{\rho}}{\partial P_{\mu}}=\frac{\partial \mathcal{P}_{\rho}}{\partial P_{\mu}} \mathcal{P}_{\mp \rho},
$$

the second term vanishes, $\mathcal{P}_{\rho}\{\ldots\} \mathcal{P}_{\sigma}=0$. Then, to the gradient approximation, $\underline{C}(x, y)$ in Eq. (4.11) may be written as 


$$
\underline{C}(x, y) \simeq\left[\sum_{\rho, \xi, \sigma= \pm} \underline{\mathcal{P}}_{\rho} \cdot \underline{A}^{\rho \xi} \cdot \underline{B}^{\xi \sigma} \cdot \underline{\mathcal{P}}_{\sigma}\right](x, y) .
$$

Thus, as in Eq. (2.15), we can use the $(2 \times 2)$ matrix notation in a "polarization space", $(\underline{\mathbf{A}})^{\rho \sigma}=\underline{A}^{\rho \sigma}(\rho, \sigma= \pm)$.

The self-energy-part $(\underline{\hat{\boldsymbol{\Sigma}}}(x, y))$ resummed propagator $\underline{\hat{\mathbf{G}}}$ obeys

$$
\begin{aligned}
& \underline{\hat{\mathbf{G}}}=\underline{\hat{\mathbf{S}}}+\underline{\hat{\mathbf{S}}} \cdot \underline{\hat{\mathbf{s}}} \cdot \underline{\hat{\mathbf{G}}}=\underline{\hat{\mathbf{S}}}+\underline{\hat{\mathbf{G}}} \cdot \underline{\hat{\mathbf{S}}} \cdot \underline{\hat{\mathbf{S}}}, \\
& \underline{\hat{\mathbf{S}}}=\left(\begin{array}{cc}
\underline{S}_{R} & 0 \\
\underline{S}_{R}-\underline{S}_{A} & -\underline{S}_{A}
\end{array}\right) \mathbf{1} \\
&-\left(\underline{S}_{R} \cdot \underline{\mathbf{f}}-\underline{\mathbf{f}} \cdot \underline{S}_{A}-\underline{\mathbf{S}}_{K}\right) \hat{M}_{+}, \\
& \underline{\mathbf{S}}_{K}= \underline{S}_{R} \cdot \gamma_{5} \underline{X} \cdot \underline{\mathbf{C}}-\underline{\mathbf{C}} \cdot \gamma_{5} \underline{X} \cdot \underline{S}_{A}, \\
& \underline{\mathbf{C}}=\left(\begin{array}{cc}
0 & \underline{C}_{+-} \\
\underline{C}_{-+} & 0
\end{array}\right) .
\end{aligned}
$$

For $\underline{\hat{\mathbf{G}}}$ and $\underline{\hat{\boldsymbol{\Sigma}}}$, we have (cf. Eqs. (2.26) - (2.30), (2.20) - (2.24))

$$
\begin{aligned}
& \hat{\hat{\mathbf{G}}}=\left(\begin{array}{cc}
\underline{\mathbf{G}}_{R} & 0 \\
\underline{\mathbf{G}}_{R}-\underline{\mathbf{G}}_{A} & -\underline{\mathbf{G}}_{A}
\end{array}\right) \\
& -\left[\underline{\mathbf{G}}_{R} \cdot \underline{\mathbf{f}}-\underline{\mathbf{f}} \cdot \underline{\mathbf{G}}_{A}-\underline{\mathbf{G}}_{K}\right] \hat{M}_{+}, \\
& \underline{\underline{\Sigma}}=\left(\begin{array}{cc}
\underline{\boldsymbol{\Sigma}}_{R} & 0 \\
-\underline{\boldsymbol{\Sigma}}_{R}+\underline{\boldsymbol{\Sigma}}_{A} & -\underline{\boldsymbol{\Sigma}}_{A}
\end{array}\right) \\
& -\left[\underline{\boldsymbol{\Sigma}}_{R} \cdot \underline{\mathbf{f}}-\underline{\mathbf{f}} \cdot \underline{\boldsymbol{\Sigma}}_{A}+\underline{\boldsymbol{\Sigma}}_{K}\right] \hat{M}_{-}, \\
& \underline{\mathbf{G}}_{K}=\underline{\mathbf{G}}_{R} \cdot \underline{\mathbf{f}}-\underline{\mathbf{f}} \cdot \underline{\mathbf{G}}_{A}+\underline{\mathbf{G}}_{12}, \\
& \underline{\boldsymbol{\Sigma}}_{K}=-\underline{\boldsymbol{\Sigma}}_{R} \cdot \underline{\mathbf{f}}+\underline{\mathbf{f}} \cdot \underline{\boldsymbol{\Sigma}}_{A}+\underline{\hat{\boldsymbol{\Sigma}}}_{12} .
\end{aligned}
$$

Eq. (4.13) may be solved to give

$$
\begin{aligned}
\underline{\mathbf{G}}_{R(A)}(x, y)= & {\left[\mathbf{1}\left(i \not \partial_{x}-m\right)^{-1} \delta^{4}(x-y)\right.} \\
& \left.-\underline{\boldsymbol{\Sigma}}_{R(A)}(x, y)\right]^{-1}, \\
\underline{\mathbf{G}}_{K}= & \underline{\mathbf{G}}_{K}^{[1]}+\underline{\mathbf{G}}_{K}^{[2]}+\underline{\mathbf{G}}_{K}^{[3]}, \\
\underline{\mathbf{G}}_{K}^{[1]}= & -\underline{\mathbf{G}}_{R} \cdot \underline{\boldsymbol{\Sigma}}_{K} \cdot \underline{\mathbf{G}}_{A},
\end{aligned}
$$




$$
\begin{aligned}
\underline{\mathbf{G}}_{K}^{[2]}= & \underline{\mathbf{G}}_{R} \cdot\left[\gamma_{5} \underline{\underline{X}} \cdot \underline{\mathbf{C}} \cdot \underline{\boldsymbol{\Sigma}}_{A}\right. \\
& \left.-\underline{\boldsymbol{\Sigma}}_{R} \cdot \underline{\mathbf{C}} \cdot \gamma_{5} \underline{\underline{X}}\right] \cdot \underline{\mathbf{G}}_{A} \\
\equiv & \underline{\mathbf{G}}_{R} \cdot \underline{\mathbf{H}} \cdot \underline{\mathbf{G}}_{A}, \\
\underline{\mathbf{G}}_{K}^{[3]}= & \underline{\mathbf{G}}_{R} \cdot \gamma_{5} \underline{\underline{X}} \cdot \underline{\mathbf{C}}-\underline{\mathbf{C}} \cdot \gamma_{5} \underline{\underline{X}} \cdot \underline{\mathbf{G}}_{A} .
\end{aligned}
$$

The form for the leading part of the DEX of $\hat{G}(X, P)(\stackrel{\mathrm{WT}}{\longleftarrow} \underline{G}(x, y))$ is the $\hat{G}$ that is deduced in Sec. II.

\section{Gradient piece of the self-energy-part resummed propagator}

$$
\text { Form for } G_{R(A)}
$$

From Eq. (4.16), we obtain, for the component $\underline{G}_{R}^{\rho \sigma}$,

$$
\begin{aligned}
\underline{G}_{R}^{\rho \rho}(x, y)= & {\left[(i \not \partial-m) \delta^{4}(x-y)-\underline{\Sigma}_{R}^{\rho \rho}\right.} \\
& \left.-\underline{\Sigma}_{R}^{\rho-\rho} \cdot \underline{G}_{R}^{(\mathrm{pre})-\rho-\rho} \cdot \underline{\Sigma}_{R}^{-\rho \rho}\right]^{-1}, \\
\underline{G}_{R}^{\rho-\rho}(x, y)= & \underline{G}_{R}^{(\mathrm{pre}) \rho \rho} \cdot \underline{\Sigma}_{R}^{\rho-\rho} \cdot \underline{G}_{R}^{-\rho-\rho} \\
= & \underline{G}_{R}^{\rho \rho} \cdot \underline{\Sigma}_{R}^{\rho-\rho} \cdot \underline{G}_{R}^{(\mathrm{pre})-\rho-\rho}, \\
\underline{G}_{R}^{(\mathrm{pre}) \rho \rho}(x, y)= & {\left[(i \not \partial-m) \delta^{4}(x-y)-\underline{\Sigma}_{R}^{\rho \rho}(x, y)\right]^{-1} . }
\end{aligned}
$$

Solving Eqs. (4.21) and (4.22), we write the solutions as $G_{R}^{\rho \sigma}=G_{R}^{(0) \rho \sigma}+G_{R}^{(1) \rho \sigma}$. Here, $G_{R}^{(0) \rho \sigma}$ is the leading part of the DEX of $G_{R}^{\rho \sigma}$, whose form has been obtained in Sec. II. The gradient part $G_{R}^{(1) \rho \sigma}$ is obtained as

$$
\begin{aligned}
& G_{R}^{(1) \rho \rho}=\frac{i}{2} G_{R}^{(0) \rho \rho}\left[\left\{\left(G_{R}^{(0) \rho \rho}\right)^{-1}, G_{R}^{(0) \rho \rho}\right\}-\left\{\Sigma_{R}^{\rho-\rho}\left|G_{R}^{(\mathrm{pre})^{-\rho-\rho}}\right| \Sigma_{R}^{-\rho \rho}\right\} G_{R}^{(0) \rho \rho}\right. \\
& \left.-\Sigma_{R}^{\rho-\rho}\left\{G_{R}^{(\mathrm{pre})-\rho-\rho}, \Sigma_{R}^{-\rho \rho}\right\} G_{R}^{(0) \rho \rho}-\left\{\Sigma_{R}^{\rho-\rho}, G_{R}^{(\mathrm{pre})^{-\rho-\rho}}\right\} \Sigma_{R}^{-\rho \rho} G_{R}^{(0) \rho \rho}\right] \\
& =\frac{i}{2}\left[\left\{G_{R}^{(0) \rho \rho},\left(G_{R}^{(0) \rho \rho}\right)^{-1}\right\}-G_{R}^{(0) \rho \rho}\left\{\Sigma_{R}^{\rho-\rho}\left|G_{R}^{(\mathrm{pre})^{-\rho-\rho}}\right| \Sigma_{R}^{-\rho \rho}\right\}\right. \\
& \left.-G_{R}^{(0) \rho \rho}\left\{\Sigma_{R}^{\rho-\rho}, G_{R}^{(\mathrm{pre})^{-\rho-\rho}}\right\} \Sigma_{R}^{-\rho \rho}-G_{R}^{(0) \rho \rho} \Sigma_{R}^{\rho-\rho}\left\{G_{R}^{(\mathrm{pre})^{-\rho-\rho}}, \Sigma_{R}^{-\rho \rho}\right\}\right] G_{R}^{(0) \rho \rho},
\end{aligned}
$$




$$
\begin{aligned}
G_{R}^{(1) \rho-\rho}= & -\frac{i}{2}\left[\left\{G_{R}^{(\mathrm{pre}) \rho \rho}, \Sigma_{R}^{\rho-\rho}\right\} G_{R}^{(0)-\rho-\rho}+\left\{G_{R}^{(\mathrm{pre}) \rho \rho}\left|\Sigma_{R}^{\rho-\rho}\right| G_{R}^{(0)-\rho-\rho}\right\}\right. \\
& \left.+G_{R}^{(\mathrm{pre}) \rho \rho}\left\{\Sigma_{R}^{\rho-\rho}, G_{R}^{(0)-\rho-\rho}\right\}\right] \\
= & -\frac{i}{2}\left[\left\{G_{R}^{(0) \rho \rho}, \Sigma_{R}^{\rho-\rho}\right\} G_{R}^{(\mathrm{pre})-\rho-\rho}+\left\{G_{R}^{(0) \rho \rho}\left|\Sigma_{R}^{\rho-\rho}\right| G_{R}^{(\mathrm{pre})-\rho-\rho}\right\}\right. \\
& \left.+G_{R}^{(0) \rho \rho}\left\{\Sigma_{R}^{\rho-\rho}, G_{R}^{(\mathrm{pre})-\rho-\rho}\right\}\right]
\end{aligned}
$$

where

$$
\{A|B| C\} \equiv \frac{\partial A}{\partial X^{\mu}} B \frac{\partial C}{\partial P_{\mu}}-\frac{\partial A}{\partial P_{\mu}} B \frac{\partial C}{\partial X^{\mu}} .
$$

Then, $\underline{G}_{R}(x, y)$ is written as (cf. Eq. (4.1))

$$
\underline{G}_{R}(x, y)=\sum_{\rho, \sigma= \pm}\left[\underline{\mathcal{P}}_{\rho} \cdot\left(G_{R}^{\rho \sigma}(P, X)\right)_{\mathrm{IWT}} \cdot \underline{\mathcal{P}}_{\sigma}\right](x, y)
$$

We write the solution to Eq. (4.23) as $G_{R}^{(\mathrm{pre}) \rho \rho}=G_{R}^{(\mathrm{pre})(0) \rho \rho}+G_{R}^{(\mathrm{pre})(1) \rho \rho}$. The form for $G_{R}^{(\text {pre) (0) } \rho \rho}$ is given in Sec. II, while the form for $G_{R}^{(\text {pre) (1) } \rho \rho}$ is given by Eq. (4.24) with the following replacements,

$$
G_{R}^{(0) \rho \sigma} \rightarrow G_{R}^{(\mathrm{pre})(0) \rho \sigma}, \quad G_{R}^{(1) \rho \sigma} \rightarrow G_{R}^{(\mathrm{pre})(1) \rho \sigma}, \quad G_{R}^{(\mathrm{pre}) \mp \rho \mp \rho} \rightarrow 0 .
$$

$G_{A}(P, X)$ is obtained from $G_{R}(P, X)$ with $\Sigma_{A}$ 's for $\Sigma_{R}$ 's.

$$
\text { Form for } \Sigma_{K} \text {, which is involved in } G_{K}^{[1]} \text { in Eq. (4.18) }
$$

Computation of Eq. (4.15) to the gradient approximation yields

$$
\begin{aligned}
& \underline{\Sigma}_{K}=\underline{L}_{c 1}+\underline{L}_{c 2}-\sum_{\rho, \sigma= \pm} \underline{\mathcal{P}}_{\rho} \cdot\left[\left(\Sigma_{R}^{\rho \sigma} f_{\sigma}\right)_{\mathrm{IWT}}-\left(f_{\rho} \Sigma_{A}^{\rho \sigma}\right)_{\mathrm{IWT}}-\underline{\Sigma}_{12}^{\rho \sigma}\right] \cdot \underline{\mathcal{P}}_{\sigma}+\Sigma_{K}^{[1]} \\
& \Sigma_{K}^{[1]}=-\frac{i}{2} \sum_{\rho, \sigma= \pm} \mathcal{P}_{\rho}\left[\left\{f_{\sigma}, \quad \Sigma_{R}^{\rho \sigma}\right\}+\left\{f_{\rho}, \quad \Sigma_{A}^{\rho \sigma}\right\}\right] \mathcal{P}_{\sigma} .
\end{aligned}
$$

$\underline{L}_{c 1}$ and $\underline{L}_{c 2}$ in Eq. (4.26) come from $\mathcal{A}_{c}$ in Eq. (4.9) (see Eq. (4.10)). The standard forms for $\Sigma_{K}^{[1]}, H$ (Eq. (4.19)), and $\gamma_{5} \underline{\underline{X}} \cdot \underline{\mathbf{C}}$ and $\underline{\mathbf{C}} \cdot \gamma_{5} \underline{\underline{X}}$ in Eq. (4.20) are given in Appendix E. 


\section{Perturbation theories - Generalized Boltzmann equations and their relatives}

The aim of this subsubsection is to construct perturbation theories. We are employing the interaction picture in the sense of [11]. Then, the quark-gluon system of our concern is characterized by a density matrix $\rho$ at an initial time $X^{0}=X_{i}^{0}$, from which $f_{\rho}\left(P, X_{i}^{0}, \vec{X}\right)$ and $C_{\rho-\rho}\left(P, X_{i}^{0}, \vec{X}\right)$ (cf. Eqs. (2.8) - (2.10)) are determined. It should be emphasized that there is no information at this stage on how $f_{\rho}(P, X)$ and $C_{\rho-\rho}(P, X)$ evolve in spacetime. Then, in the course of construction of a perturbative framework, certain evolution equations that describe the spacetime evolution for $f_{\rho}$ and $C_{\rho-\rho}$ should be settled. As a matter of fact, one can choose any forms for the evolution equations, on the basis of which a perturbative framework is constructed. Different frameworks are physically equivalent in the sense that they lead to the same result for the physical quantities (see below for more details). In the sequel, we construct two kind of perturbative frameworks by employing two different forms for the evolution equations.

As seen from Eq. (4.14), the propagator $\hat{G}$ is written in terms of $G_{R}, G_{A}$, and $G_{K}$. $G_{R}(P, X)\left[G_{A}(P, X)\right]$ is analytic in an upper [a lower] half complex $p_{0}$-plane. Then, in calculating some quantity, the parts of $\hat{G}$ that are proportional to $G_{R}$ or to $G_{A}$ yield welldefined contributions. Now, we observe that $G_{K}^{[1]}$ and $G_{K}^{[2]}$, Eqs. (4.18) and (4.19), contain $G_{R} G_{A}$. Since $G_{A}$ is essentially the complex conjugate of $G_{R}, G_{R} G_{A}$ is disastrously large on the energy shells ${ }^{2}, p_{0}= \pm \omega_{ \pm}( \pm \vec{p}, X)$, on which

$$
\left.\operatorname{Re}\left(G_{R}^{\rho \rho}(P, X)\right)^{-1}\right|_{p_{0}= \pm \omega_{ \pm}( \pm \vec{p}, X)}=0
$$

As a matter of fact, in the narrow-width approximation, $\operatorname{Im}\left(G_{R}^{\rho \rho}\right)^{-1} \rightarrow \epsilon\left(p_{0}\right) 0^{+}, G_{R}^{\rho \rho} G_{A}^{\rho \rho}$ develops pinch singularities at the energy shells in a $p_{0}$-plane. ${ }^{3}$ Then, $G_{K}^{[1]}$ and $G_{K}^{[2]}$ yield diverging contribution. In practice, $\operatorname{Im}\left(G_{R}^{\rho \rho}\right)^{-1}\left(\propto g^{2}\right)$ is a small quantity, so that the contri-

\footnotetext{
${ }^{2}$ How to find the solution to Eq. (4.28) is given in Appendix F.

${ }^{3}$ This is a characteristic feature of nonequilibrium dynamics [12].
} 
butions, although not divergent, are large, which invalidates the perturbative scheme. These large contributions come from the vicinities of the energy shells, on which $\operatorname{Re}\left(G_{R}^{\rho \rho}\right)^{-1} \sim 0$.

Appropriate use of the first and second equalities of Eq. (2.36) together with Eq. (2.31) shows that $G_{R}^{\rho \rho} G_{A}^{ \pm \rho \mp \rho}$ and $G_{R}^{\rho-\rho} G_{A}^{ \pm \rho \mp \rho}$ do not yield large contributions. This is because, in general, the energy shells of $G_{R}^{\rho \rho}$ and $G_{R}^{-\rho-\rho}$, and of $G_{R}^{(\text {pre }) \rho \rho}$ and $G_{R}^{(\text {pre })-\rho-\rho}$, do not coincide. For the case of $f_{+}=f_{-}$, however, this is not the case. For $G_{R}^{\rho \rho} G_{A}^{ \pm \rho \mp \rho}$, a large contribution emerges from the region $\operatorname{Re}\left(G_{R}^{\rho \rho}\right)^{-1} \sim 0$, and, for $G_{R}^{\rho-\rho} G_{A}^{ \pm \rho \mp \rho}$, large contributions emerge from the regions $\operatorname{Re}\left(G_{R}^{\rho \rho}\right)^{-1} \sim 0$ and from the region $\operatorname{Re}\left(G_{R}^{(\mathrm{pre}) \rho \rho}\right)^{-1} \sim 0$.

From Eqs. (4.18) and (4.19) with Eq. (4.26), we have for the $(\rho \rho)$ - and $(\rho-\rho)$-components

$$
\begin{aligned}
&(\rho= \pm) \text { of } \underline{H}-\underline{\Sigma}_{K}\left(=\underline{G}_{R}^{-1} \cdot\left(\underline{G}_{K}^{[1]}+\underline{G}_{K}^{[2]}\right) \cdot \underline{G}_{A}^{-1}\right), \\
& \underline{H}^{\rho \rho}-\underline{\Sigma}_{K}^{\rho \rho} \underline{\mathrm{WT}} \rightarrow-\left.i\left[\frac{P \cdot \partial_{X}}{P^{2}} \not P+\frac{N \cdot \partial_{X}}{N^{2}} \not X+\frac{\rho \epsilon\left(p_{0}\right)}{e_{\perp}^{2}}\left(e_{\perp} \cdot \partial_{X}\right) \not P X\right]\right] f_{\rho}+i \tilde{\Gamma}_{p}^{\rho \rho}-\Sigma_{B}^{\rho \rho}, \\
& \underline{H}^{\rho-\rho}-\underline{\Sigma}_{K}^{\rho-\rho} \stackrel{\mathrm{WT}}{\longrightarrow}--i \gamma_{5}\left[\left\{\frac{m}{P^{2}}\left(N \cdot \partial_{X}\right)+\frac{\rho \epsilon\left(p_{0}\right)}{P^{2}}\left(e_{\perp} \cdot \partial_{X}\right)\right\} \not P\right. \\
&-\left\{\frac{\rho \epsilon\left(p_{0}\right)}{N^{2}} e_{\perp}^{\mu} \frac{\partial N_{\mu}}{\partial P_{\alpha}}+\frac{m}{2 N^{2}} \frac{\partial N^{2}}{\partial P_{\alpha}}\right\} \not X \frac{\partial}{\partial X^{\alpha}} \\
&\left.-\left\{\frac{1}{2 N^{2}} \frac{\partial N^{2}}{\partial P_{\alpha}} \frac{\partial}{\partial X^{\alpha}}-m \frac{\rho \epsilon\left(p_{0}\right)}{e_{\perp}^{2}} e_{\perp}^{\mu} \frac{\partial N^{\mu}}{\partial P_{\alpha}} \frac{\partial}{\partial X^{\alpha}}-\frac{P \cdot \partial_{X}}{P^{2}}\right\} \not P D X\right] C_{\rho-\rho} \\
&+i \tilde{\Gamma}_{p}^{\rho-\rho}-\Sigma_{B}^{\rho-\rho}, \\
& \tilde{\Gamma}_{p}^{\rho \sigma}= i\left[\left(1-f_{\sigma}\right) \Sigma_{12}^{\rho \sigma}+f_{\rho} \Sigma_{21}^{\rho \sigma}+\left(f_{\rho}-f_{\sigma}\right) \Sigma_{11}^{\rho \sigma}\right] \\
& \Sigma_{B}^{\rho \sigma}= \Sigma_{K}^{[1] \rho \sigma}-\left[\left(\gamma_{5} \underline{X} \underline{C} \cdot \underline{\Sigma_{A}}-\underline{\Sigma}_{R} \cdot \underline{C} \cdot \gamma_{5} \underline{\underline{X}}\right)^{\rho \sigma}\right]_{\mathrm{WT}} .
\end{aligned}
$$

The first term on the R.H.S. of Eq. (4.29) (Eq. (4.30)) comes from the counter Lagrangian $\underline{L}_{c 1}\left(\underline{L}_{c 2}\right)$, Eq. (4.6) (Eq. (4.7)), in Eq. (4.26).

For later reference, we note that the physical number densities, $N_{ \pm}^{(\mathrm{ph})}(P, X)$ and $\bar{N}_{ \pm}^{(\text {ph })}(P, X)$, are obtained through computing current density,

$$
\begin{aligned}
\left\langle j^{\mu}(x)\right\rangle & \equiv \operatorname{Tr}\left[\bar{\psi}(x) \gamma^{\mu} \psi(x) \rho\right] \\
& =-\frac{i}{2} \operatorname{Tr}\left\{\gamma^{\mu}\left[\underline{G}_{21}(x, x)+\underline{G}_{12}(x, x)\right] \rho\right\} .
\end{aligned}
$$

Similarly, the physical $C_{ \pm \mp}(P, X), C_{ \pm \mp}^{(\mathrm{ph})}(P, X)$, is obtained from 


$$
\begin{aligned}
\left\langle j_{5}^{\mu}(x)\right\rangle & \equiv \operatorname{Tr}\left[\bar{\psi}(x) \gamma_{5} \gamma^{\mu} \psi(x) \rho\right] \\
& =-\frac{i}{2} \operatorname{Tr}\left\{\gamma_{5} \gamma^{\mu}\left[\underline{G}_{21}(x, x)+\underline{G}_{12}(x, x)\right] \rho\right\} .
\end{aligned}
$$

\section{Bare- $N$ scheme}

As has been emphasized at the beginning of this subsubsection, $f_{\rho}(P, X)$ in Eq. (4.29) and $C_{\rho-\rho}(P, X)$ in Eq. (4.30) $\left(X_{i}^{0}<X^{0}\right)$ have not been defined so far. For the purpose of determining them, we impose here the condition that the counter Lagrangian $L_{c}$ is absent, $L_{c}=0:$

$$
\begin{aligned}
P \cdot \partial_{X} f_{\rho}^{(\mathrm{B})} & =N \cdot \partial_{X} f_{\rho}^{(\mathrm{B})}=e_{\perp} \cdot \partial_{X} f_{\rho}^{(\mathrm{B})}=0, \\
{\left[m\left(N \cdot \partial_{X}\right)\right.} & \left.+\rho \epsilon\left(p_{0}\right)\left(e_{\perp} \cdot \partial_{X}\right)\right] C_{\rho-\rho}^{(\mathrm{B})}=\left[\rho \epsilon\left(p_{0}\right) e_{\perp}^{\mu} \frac{\partial N_{\mu}}{\partial P_{\alpha}}+\frac{m}{2} \frac{\partial N^{2}}{\partial P_{\alpha}}\right] \frac{\partial C_{\rho-\rho}^{(\mathrm{B})}}{\partial X^{\alpha}} \\
& =\left[\frac{P^{2}}{2} \frac{\partial N^{2}}{\partial P_{\alpha}} \frac{\partial}{\partial X^{\alpha}}+m \rho \epsilon\left(p_{0}\right) e_{\perp}^{\mu} \frac{\partial N^{\mu}}{\partial P_{\alpha}} \frac{\partial}{\partial X^{\alpha}}-N^{2}\left(P \cdot \partial_{X}\right)\right] C_{\rho-\rho}^{(\mathrm{B})}=0,
\end{aligned}
$$

where we have written $f_{\rho}^{(\mathrm{B})}\left(C_{\rho-\rho}^{(\mathrm{B})}\right)$ for $f_{\rho}\left(C_{\rho-\rho}\right)$. Then, Eq. (4.29) (Eq. (4.30)), of which the first term on the RHS is absent, is to be solved under the given initial data $f_{\rho}\left(P, X_{i}^{0}, \vec{X}\right)\left(C_{\rho-\rho}\left(P, X_{i}^{0}, \vec{X}\right)\right)$. Eq. (4.35) is a "free Boltzmann equation" and its relatives for the "bare" number densities, $N_{\rho}^{(\mathrm{B})}\left(p_{0}, \vec{p}, X\right)=\theta\left(p_{0}\right) f_{\rho}^{(\mathrm{B})}(P, X)$ and $\bar{N}_{\rho}^{(\mathrm{B})}\left(\left|p_{0}\right|, \vec{p}, X\right)=$ $1-\theta\left(-p_{0}\right) f_{\rho}^{(\mathrm{B})}\left(p_{0},-\vec{p}, X\right)$, (cf. Eq. $\left.(2.12)\right)$.

The physical number densities, which are obtained from $\left\langle j^{\mu}(x)\right\rangle$ (Eq. (4.33)), and the physical $C_{\rho-\rho}^{(\mathrm{ph})}$, which is obtained from $\left\langle j_{5}^{\mu}(x)\right\rangle($ Eq. $(4.34))$, are functionals of $f_{\sigma}^{(\mathrm{B})}$ and $C_{\sigma-\sigma}^{(\mathrm{B})}$ :

$$
\begin{aligned}
f_{\rho}^{(\mathrm{ph})}(P, X)= & \theta\left(p_{0}\right) N_{\rho}^{(\mathrm{ph})}\left(p_{0}, \vec{p}\right) \\
& +\theta\left(-p_{0}\right)\left[1-\bar{N}_{\rho}^{(\mathrm{ph})}\left(\left|p_{0}\right|,-\vec{p}\right)\right] \\
= & \mathcal{F}_{\rho}\left(P, X ;\left[f_{\sigma}^{(\mathrm{B})}\right],\left[C_{\sigma-\sigma}^{(\mathrm{B})}\right]\right), \\
C_{\rho-\rho}^{(\mathrm{ph})}(P, X)= & \mathcal{G}_{\rho}\left(P, X ;\left[f_{\sigma}^{(\mathrm{B})}\right],\left[C_{\sigma-\sigma}^{(\mathrm{B})}\right]\right) .
\end{aligned}
$$

$\mathcal{F}_{\rho}$ and $\mathcal{G}_{\rho}$ here contain large contributions mentioned above. Solving these equations for $f_{\rho}^{(\mathrm{B})}$ and $C_{\rho-\rho}^{(\mathrm{B})}$, , one obtains 


$$
\begin{aligned}
f_{\rho}^{(\mathrm{B})} & =f_{\rho}^{(\mathrm{B})}\left(P, X ;\left[f_{\sigma}^{(\mathrm{ph})}\right],\left[C_{\sigma-\sigma}^{(\mathrm{ph})}\right]\right), \\
C_{\rho-\rho}^{(\mathrm{B})} & =C_{\rho-\rho}^{(\mathrm{B})}\left(P, X ;\left[f_{\sigma}^{(\mathrm{ph})}\right],\left[C_{\sigma-\sigma}^{(\mathrm{ph})}\right]\right) .
\end{aligned}
$$

In the case of scalar theory [8], the physical number density is shown to obey the generalized Boltzmann equation.

Computation of some physical quantity yields the expression $F\left(\left[f_{\rho}^{(\mathrm{B})}\right],\left[C_{\rho-\rho}^{(\mathrm{B})}\right]\right)$, which includes large contribution. Substituting the RHS's of Eqs. (4.37) and of (4.38) for, in

respective order, $f_{\rho}^{(\mathrm{B})}$ and $C_{\rho-\rho}^{(\mathrm{B})}$ in $F$, one obtains the expression $F^{\prime}\left(\left[f_{\rho}^{(\mathrm{ph})}\right],\left[C_{\rho-\rho}^{(\mathrm{ph})}\right]\right)$, which does not include large contribution.

The perturbation theory thus constructed is called the "bare- $N$ scheme" in [8].

\section{Physical-N scheme}

Here we aim at constructing a perturbation theory, on the basis of which no large contribution appear. Then, in such a scheme, there are no large terms in the relations between $\left(f_{\rho}^{(\mathrm{ph})}, C_{\rho-\rho}^{(\mathrm{ph})}\right)$ and $\left(f_{\rho}, C_{\rho-\rho}\right)$. This is achieved if the condition, Eq. $(4.29)=$ Eq. $(4.30)=0$, could be imposed. This is, however, not possible. Nevertheless, it is possible to construct the scheme that is free from the large contributions.

For determining so far arbitrary $f_{\rho}(P, X)$ and $C_{\rho-\rho}(P, X)\left(X_{i}^{0}<X^{0}\right)$, we impose the conditions,

$$
\begin{aligned}
& \operatorname{Tr}\left(\not P+\Omega_{f}(P, X)\right)(\text { Eq. (4.29)) }=\operatorname{Tr} \not \mathcal{X}(\text { Eq. (4.29)) } \\
& =\operatorname{Tr} \not P D T(\text { Eq. }(4.29))=0 \text {, } \\
& \operatorname{Tr} \gamma_{5}(\not P-m) \not \mathcal{N}(\text { Eq. (4.30)) } \\
& =\operatorname{Tr} \gamma_{5}\left(\not P-\Omega_{C}(P, X)\right)(\text { Eq. (4.30)) } \\
& =\operatorname{Tr} \gamma_{5} \mathcal{X}(\text { Eq. }(4.30))=0 \text {. }
\end{aligned}
$$

Here, $\Omega_{f}(P, X)$ and $\Omega_{C}(P, X)$ are arbitrary functions with the property,

$$
\Omega_{f}\left(p_{0}= \pm \omega_{ \pm}( \pm \vec{p}, X), \vec{p}, X\right)
$$




$$
\begin{aligned}
& =\Omega_{C}\left(p_{0}= \pm \omega_{ \pm}( \pm \vec{p}, X), \vec{p}, X\right) \\
& =\left[\left(\omega_{ \pm}( \pm \vec{p}, X)\right)^{2}-\vec{p}^{2}\right]^{1 / 2} .
\end{aligned}
$$

As has been discussed in [8], this arbitrariness does not matter (see also subsubsection 6 below). Computation of Eqs. (4.39) and (4.40) yields, in respective order,

$$
\begin{aligned}
P \cdot \partial_{X} f_{\rho} & =\frac{1}{4} \operatorname{Tr}\left(\not P+\Omega_{f}(P, X)\right)\left[\tilde{\Gamma}_{p}^{\rho \rho}+i \Sigma_{B}^{\rho \rho}\right], \\
N \cdot \partial_{X} f_{\rho} & =\frac{1}{4} \operatorname{Tr} \not T\left[\tilde{\Gamma}_{p}^{\rho \rho}+i \Sigma_{B}^{\rho \rho}\right] \\
e_{\perp} \cdot \partial_{X} f_{\rho} & =\frac{1}{4} \rho \epsilon\left(p_{0}\right) \operatorname{Tr} \not P \mathcal{X}\left[\tilde{\Gamma}_{p}^{\rho \rho}+i \Sigma_{B}^{\rho \rho}\right]
\end{aligned}
$$

and

$$
\begin{gathered}
{\left[N^{2} P \cdot \partial_{X}-\frac{1}{2}\left(P^{2}-m^{2}\right) \frac{\partial N^{2}}{\partial P_{\alpha}} \frac{\partial}{\partial X^{\alpha}}\right] C_{\rho-\rho}} \\
=-\frac{1}{4} \operatorname{Tr} \gamma_{5}(\not P-m) \mathcal{X}\left[\tilde{\Gamma}_{p}^{\rho-\rho}+i \Sigma_{B}^{\rho-\rho}\right] \\
{\left[m N \cdot \partial_{X}+\rho \epsilon\left(p_{0}\right) e_{\perp} \cdot \partial_{X}\right] C_{\rho-\rho}} \\
=-\frac{1}{4} \operatorname{Tr} \gamma_{5}\left(\not P-\Omega_{C}(P, X)\right)\left[\tilde{\Gamma}_{p}^{\rho-\rho}+i \Sigma_{B}^{\rho-\rho}\right] \\
{\left[\rho \epsilon\left(p_{0}\right) e_{\perp}^{\mu} \frac{\partial N_{\mu}}{\partial P_{\alpha}} \frac{\partial}{\partial X^{\alpha}}+\frac{m}{2} \frac{\partial N^{2}}{\partial P_{\alpha}} \frac{\partial}{\partial X^{\alpha}}\right] C_{\rho-\rho}} \\
=\frac{1}{4} \operatorname{Tr} \gamma_{5} X^{X}\left[\tilde{\Gamma}_{p}^{\rho-\rho}+i \Sigma_{B}^{\rho-\rho}\right]
\end{gathered}
$$

These equations are the determining equations for $f_{\rho}$ and $C_{\rho-\rho}$, which are to be solved under the given initial data, $f_{\rho}\left(P, X_{i}^{0}, \vec{X}\right)$ and $C_{\rho-\rho}\left(P, X_{i}^{0}, \vec{X}\right)$, respectively.

After imposition of Eqs. (4.42) - (4.47), $H^{\rho \pm \rho}-\Sigma_{K}^{\rho \pm \rho}$, Eqs. (4.29) and (4.30), turns out to

$$
\begin{aligned}
H^{\rho \rho}-\Sigma_{K}^{\rho \rho}= & \frac{i}{4 P^{2}}\left(P^{2}-\Omega_{f} \not P\right) \operatorname{Tr}\left(\tilde{\Gamma}_{p}^{\rho \rho}+i \Sigma_{B}^{\rho \rho}\right) \\
H^{\rho-\rho}-\Sigma_{K}^{\rho-\rho}= & \frac{i}{4 P^{2}} \gamma_{5}\left(P^{2}-\Omega_{C} \not P\right) \\
& \times \operatorname{Tr} \gamma_{5}\left(\tilde{\Gamma}_{p}^{\rho-\rho}+i \Sigma_{B}^{\rho-\rho}\right)
\end{aligned}
$$


On the energy shells $p_{0}= \pm \omega_{ \pm}$, these quantities vanish, since $\left(P^{2}-\Omega_{f(C)} \not P\right)\left(P^{2}+\Omega_{f(C)} \not P\right)=$ $P^{2}\left(P^{2}-\Omega_{f(C)}^{2}\right)$. Then, the above mentioned large contributions, which turn out to be diverging contributions in the narrow-width approximation, do not appear. Thus, $G_{K}^{[1]}+$ $G_{K}^{[2]}$ turns out to be a well-behaved function. As a matter of fact, in the narrow-width approximation,

$$
\begin{gathered}
G_{K}^{[2]}+G_{K}^{[2]} \propto \frac{p_{0} \mp \omega_{ \pm}}{\left(p_{0} \mp \omega_{ \pm}\right)^{2}+\left(0^{+}\right)^{2}}=\frac{\mathbf{P}}{p_{0} \mp \omega_{ \pm}} \\
\left(p_{0} \simeq \pm \omega_{ \pm}\right),
\end{gathered}
$$

which is a well-defined distribution. In particular, the relations between the physical $\left(f_{\rho}^{(\mathrm{ph})}, C_{\rho-\rho}^{(\mathrm{ph})}\right)$ and $\left(f_{\rho}, C_{\rho-\rho}\right)$ contain no large term:

$$
f_{\rho}^{(\mathrm{ph})}=f_{\rho}+\Delta f_{\rho}, \quad C_{\rho-\rho}^{(\mathrm{ph})}=C_{\rho-\rho}+\Delta C_{\rho-\rho}
$$

with $\Delta f_{\rho}$ and $\Delta C_{\rho-\rho}$ the perturbative corrections.

Proceeding as in [9], from Eq. (4.42) on the energy shells, one obtains a generalized Boltzmann equation. In fact, the term with $\tilde{\Gamma}_{p}^{\rho \rho}$ on the RHS of Eq. (4.42) is proportional to the net production rate. To avoid complete repetition, we do not reproduce it here.

\section{Discussion}

Here we like to mention a similarity between the two schemes presented here, the bare$N$ scheme and the physical- $N$ scheme, and those in the ultra-violet (UV) renormalization scheme in quantum-field theory. For simplicity of presentation, taking a complex-scalar theory, we focus on the mass renormalization and do not mention on the coupling constantand wave function-renormalizations.

Summary of the UV-renormalization theory

"Bare" UV renormalization scheme: The free Lagrangian density reads $\mathcal{L}_{0}=$ $-\phi^{\dagger}(x)\left(\partial^{2}+m_{B}^{2}\right) \phi(x)$ with $m_{B}$ the bare mass. Computation of the physical mass $M_{\mathrm{ph}}$ 
yields $M_{\mathrm{ph}}=M_{\mathrm{ph}}\left(m_{B}\right)$, which includes diverging terms. Solving this equation for $m_{B}$, we have $m_{B}=m_{B}\left(M_{\mathrm{ph}}\right)$. Perturbative computation of some physical quantity $F$ yields the expression $F=F\left(m_{B}\right)$, which contains, in general, UV-divergences. Substituting the equation $m_{B}=m_{B}\left(M_{\mathrm{ph}}\right)$ for $m_{B}$ in $F\left(m_{B}\right)$, one gets $F_{R}\left(M_{\mathrm{ph}}\right) \equiv F\left(m_{B}\left(M_{\mathrm{ph}}\right)\right)$, which is free from UV-divergence.

Physical UV renormalization scheme: One introduces new free Lagrangian $\mathcal{L}_{0}^{\prime}=$ $-\phi^{\dagger}(x)\left(\partial^{2}+m^{2}\right) \phi(x)$ with $m$ the renormalized mass. Then, the counter Lagrangian should be introduced, $\mathcal{L}_{c}=\mathcal{L}_{m}-\mathcal{L}_{m}^{\prime}=\phi^{\dagger}(x)\left[m^{2}-m_{B}^{2}\right] \phi(x) . m^{2}-m_{B}^{2}$ is determined so that the perturbatively computed physical mass $M_{\mathrm{ph}}$ is free from the UV-divergence. Thus, no diverging term is involved in the relation $M_{\mathrm{ph}}=M_{\mathrm{ph}}(m)$. However, there is arbitrariness in the definition of the finite part of $m$, which is determined by imposing some condition. This arbitrariness is called a "renormalization scheme dependence" (see, e.g., [13]). It is well known that, when one computes some physical quantity $F$ up to, say, $n$th order of perturbation theory, the above arbitrariness affects $F$ at the next to the $n$th order. The renormalization scheme, in which $m=M^{(\mathrm{ph})}$, is convenient for many cases.

\section{Summary of the two schemes presented above}

Bare- $N$ scheme: No counter Lagrangian is introduced. Computation of the physical number densities (that are related to $f_{\rho}^{(\mathrm{ph})}$ ) and $C_{\rho-\rho}^{(\mathrm{ph})}$, which are the functionals of $f_{\rho}^{(\mathrm{B})}$ and $C_{\rho-\rho}^{(\mathrm{B})}$, include large contributions. Perturbative computation of some quantity yields the expression, which is written in terms of $f_{\rho}^{(\mathrm{B})}$ and $C_{\rho-\rho}^{(\mathrm{B})}$ and includes large contributions.

Rewriting it in terms of the physical quantities, $f_{\rho}^{(\mathrm{ph})}$ and $C_{\rho-\rho}^{(\mathrm{ph})}$, one obtains the largecontribution free form.

Physical-N scheme: We introduce a counter Lagrangian $\underline{L}_{c}=\underline{L}_{c 1}+\underline{L}_{c 2}$, which is determined so that the perturbatively computed physical number densities and $C_{\rho-\rho}^{(\mathrm{ph})}$ do not contain large contributions. There is arbitrariness in the definition of the "finite parts" of $f_{\rho}$ and $C_{\rho-\rho}$. The arbitrariness in the choice of the functions $\Omega_{f}$ (Eqs. (4.42) and (4.48)) and 
$\Omega_{C}$ (Eqs. (4.46) and (4.49)) is this arbitrariness. It is worth mentioning that, if we could choose $\Omega_{f}$ and $\Omega_{C}$ so that $f_{\rho}(P, X)=f_{\rho}^{(\mathrm{ph})}(P, X)$, Eq. (4.42) on the energy-shell turns out to a genuine (generalized) Boltzmann equation. In the opposite case, the function that obey the generalized Boltzmann equation is $f_{\rho}$ and the physical $f_{\rho}^{(\mathrm{ph})}$ is written as in Eq. (4.50).

Similar comment to the above one at the end of the Physical UV renormalization scheme may be made here.

\section{Correspondence}

Above observation discloses the correspondence between the two schemes presented here and those in the UV-renormalization scheme:

Bare scheme:

$$
\begin{aligned}
& \int d^{4} x \mathcal{L}_{0}(x) \leftrightarrow \mathcal{A}_{0} \text { in Eq. (4.8) with } \underline{L}_{c}=0, \\
& m_{B} \leftrightarrow f_{\rho}^{(\mathrm{B})} \text { and } C_{\rho-\rho}^{(\mathrm{B})}, \\
& M_{\mathrm{ph}} \leftrightarrow f_{\rho}^{(\mathrm{ph})} \text { and } C_{\rho-\rho}^{(\mathrm{ph})}
\end{aligned}
$$

Physical scheme:

$$
\begin{aligned}
& \int d^{4} x \mathcal{L}_{0}^{\prime}(x) \leftrightarrow \mathcal{A}_{0}, \\
& \int d^{4} x \mathcal{L}_{c}(x) \leftrightarrow \mathcal{A}_{c} \text { in Eq. }(4.9), \\
& m \leftrightarrow f_{\rho} \text { and } C_{\rho-\rho}, \\
& M_{\mathrm{ph}} \leftrightarrow f_{\rho}^{(\mathrm{ph})} \text { and } C_{\rho-\rho}^{(\mathrm{ph})},
\end{aligned}
$$

Absence of

$$
\text { divergence } \leftrightarrow \text { large contribution, }
$$

Arbitrariness in $m \leftrightarrow$ Arbitrariness in $\Omega_{f}$ and $\Omega_{C}$,

Scheme with

$$
m=M^{(\mathrm{ph})} \leftrightarrow f_{\rho}=f_{\rho}^{(\mathrm{ph})} \text { and } C_{\rho-\rho}=C_{\rho-\rho}^{(\mathrm{ph})} .
$$




\section{B. Gluon sector}

\section{Preliminary}

Configuration-space counterparts of Eqs. (3.6) and (3.7) are, with obvious notation,

$$
\underline{A}^{\mu \nu}(x, y)=\sum_{U, V=T, L, G} \sum_{j=1}^{J_{U V}}\left[\underline{\mathcal{R}}_{L j}^{U V} \cdot \underline{\tilde{A}}_{j}^{U V} \cdot \underline{\mathcal{R}}_{R j}^{U V}\right]^{\mu \nu}(x, y),
$$

where $J_{T T}=4, J_{L L}=J_{G G}=J_{L G}=J_{G L}=1$ and $J_{T L}=J_{L T}=J_{T G}=J_{G T}=2$, and

$$
\begin{aligned}
& \sum_{j=1}^{4}\left[\underline{\mathcal{\mathcal { R }}}_{j}^{T T} \cdot \underline{\tilde{A}}_{j}^{T T} \cdot \underline{\mathcal{\mathcal { R }}}_{j}^{T T}\right]^{\mu \nu} \equiv \underline{\mathcal{P}}_{T}^{\mu \rho} \cdot \underline{A}_{1}^{T T} \cdot\left(\underline{\mathcal{P}}_{T}\right)_{\rho}^{\nu}+\underline{\tilde{\zeta}}^{\mu} \cdot \underline{A}_{2}^{T T} \cdot \underline{\tilde{\zeta}}^{\nu} \\
& -\underline{\tilde{\zeta}}^{\mu} \cdot \underline{A}_{3}^{T T^{\prime}} \cdot \underline{E}_{\perp}^{\nu}+\underline{E}_{\perp}^{\mu} \cdot \underline{A}_{3}^{T^{\prime} T} \cdot \underline{\tilde{\zeta}}^{\nu}, \\
& {\left[\underline{\mathcal{R}}_{j}^{L L} \cdot \underline{\tilde{A}}_{j}^{L L} \cdot \underline{\mathcal{R}}_{j}^{L L}\right]^{\mu \nu} \equiv n^{\mu} \underline{A}_{1}^{L L} n^{\nu},} \\
& {\left[\underline{\mathcal{R}}_{j}^{G G} \cdot \underline{\tilde{A}}_{j}^{G G} \cdot \underline{\mathcal{R}}_{j}^{G G}\right]^{\mu \nu} \equiv\left(i \tilde{\partial}^{\mu}\right) \underline{A}_{1}^{G G}\left(i \tilde{\partial}^{\nu}\right),} \\
& \sum_{j=1}^{2}\left[\underline{\mathcal{R}}_{j}^{T L} \cdot \underline{\tilde{A}}_{j}^{T L} \cdot \underline{\mathcal{R}}_{j}^{T L}\right]^{\mu \nu} \equiv \underline{\tilde{\zeta}}^{\mu} \cdot \underline{A}_{1}^{T L} n^{\nu}+\underline{E}_{\perp}^{\mu} \cdot \underline{A}_{2}^{T L} n^{\nu}, \\
& \sum_{j=1}^{2}\left[\underline{\mathcal{R}}_{j}^{L T} \cdot \underline{\tilde{A}}_{j}^{L T} \cdot \underline{\mathcal{R}}_{j}^{L T}\right]^{\mu \nu} \equiv n^{\mu} \underline{A}_{1}^{L T} \cdot \underline{\tilde{\zeta}}^{\nu}-n^{\mu} \underline{A}_{2}^{L T} \cdot \underline{E}_{\perp}^{\nu}, \\
& \sum_{j=1}^{2}\left[\underline{\mathcal{R}}_{j}^{T G} \cdot \underline{\tilde{A}}_{j}^{T G} \cdot \underline{\mathcal{R}}_{j}^{T G}\right]^{\mu \nu} \equiv \underline{E}_{\perp}^{\mu} \cdot \underline{A}_{1}^{T G}\left(i \tilde{\partial}^{\nu}\right)+\underline{\tilde{\zeta}}^{\mu} \cdot \underline{A}_{2}^{T G}\left(i \tilde{\partial}^{\nu}\right), \\
& \sum_{j=1}^{2}\left[\underline{\mathcal{R}}_{j}^{G T} \cdot \underline{\tilde{A}}_{j}^{G T} \cdot \underline{\mathcal{\mathcal { R }}}_{j}^{G T}\right]^{\mu \nu} \equiv\left(i \tilde{\partial}^{\mu}\right) \underline{A}_{1}^{G T} \cdot \underline{E}_{\perp}^{\nu}-\left(i \tilde{\partial}^{\mu}\right) \underline{A}_{2}^{G T} \cdot \underline{\tilde{\zeta}}^{\nu} \\
& {\left[\underline{\mathcal{R}}_{j}^{L G} \cdot \underline{\tilde{A}}_{j}^{L G} \cdot \underline{\mathcal{R}}_{j}^{L G}\right]^{\mu \nu} \equiv n^{\mu} \underline{A}_{1}^{L G}\left(i \tilde{\partial}^{\nu}\right),} \\
& {\left[\underline{\mathcal{R}}_{j}^{G L} \cdot \underline{\tilde{A}}_{j}^{G L} \cdot \underline{\mathcal{R}}_{j}^{G L}\right]^{\mu \nu} \equiv-\left(i \tilde{\partial}^{\mu}\right) \underline{A}_{1}^{G L} n^{\nu},}
\end{aligned}
$$

with $\tilde{\partial}^{\mu}=\partial^{\mu}-n^{\mu} \partial_{0}$ and $\partial_{0}=\partial / \partial X_{0}$.

\section{Bare propagator and counter-Lagrangian}

We proceed as in [10]. We start from the expression for the bare propagator $\underline{\hat{D}}(x, y)$ (cf.

Eqs. (3.10) - (3.13)), 


$$
\begin{aligned}
& \underline{\hat{D}}^{\mu \nu}=\left(\underline{\mathcal{P}}_{T} \cdot \underline{\hat{D}}_{T} \cdot \underline{\mathcal{P}}_{T}\right)^{\mu \nu}+\left(\underline{\mathcal{P}}_{L} \cdot \underline{\hat{D}}_{L} \cdot \underline{\mathcal{P}}_{L}\right)^{\mu \nu}+i \tilde{\partial}^{\mu} \underline{\hat{D}}_{G}\left(i \tilde{\partial}^{\nu}\right) \\
& +n^{\mu} \underline{\underline{D}}_{L T}\left(i \tilde{\partial}^{\nu}\right)+i \tilde{\partial}^{\mu} \underline{\underline{D}}_{T L} n^{\nu}+\underline{D}_{K} \hat{M}_{+}, \\
& \underline{\hat{D}}_{T}=\left(\begin{array}{cc}
-\underline{\Delta}_{R} & 0 \\
-\underline{\Delta}_{R}+\underline{\Delta}_{A} & \underline{\Delta}_{A}
\end{array}\right)-\left[\underline{\Delta}_{R} \cdot \underline{\tilde{f}}-\underline{\tilde{f}} \cdot \underline{\Delta}_{A}\right] \hat{M}_{+}, \\
& \underline{D}_{L}=-\left[\frac{1}{\tilde{P}^{2}}\left(1+\lambda \frac{p_{0}^{2}}{\tilde{P}^{2}}\right)\right]_{\mathrm{IFT}} \hat{\tau}_{3} \\
& -\left[\left\{\frac{1}{\tilde{P}^{2}}\left(1+\lambda \frac{p_{0}^{2}}{\tilde{P}^{2}}\right)\right\}_{\mathrm{IFT}} \cdot \underline{\tilde{f}}-\underline{\tilde{f}} \cdot\left\{\frac{1}{\tilde{P}^{2}}\left(1+\lambda \frac{p_{0}^{2}}{\tilde{P}^{2}}\right)\right\}_{\mathrm{IFT}}\right] \hat{M}_{+}, \\
& \underline{\mathcal{D}}_{G}=-\lambda\left[\frac{1}{\tilde{P}^{4}}\right]_{\mathrm{IFT}} \hat{\tau}_{3}-\lambda\left[\left\{\frac{1}{\tilde{P}^{4}}\right\}_{\mathrm{IFT}} \cdot \underline{\tilde{f}}-\underline{\tilde{f}} \cdot\left\{\frac{1}{\tilde{P}^{4}}\right\}_{\mathrm{IFT}}\right] \hat{M}_{+}, \\
& \underline{D}_{L T}=\underline{\mathcal{D}}_{T L}=-\lambda\left[\frac{p_{0}}{\tilde{P}^{4}}\right]_{\mathrm{IFT}} \hat{\tau}_{3}-\lambda\left[\left\{\frac{p_{0}}{\tilde{P}^{4}}\right\}_{\mathrm{IFT}} \cdot \underline{\tilde{f}}-\underline{\tilde{f}} \cdot\left\{\frac{p_{0}}{\tilde{P}^{4}}\right\}_{\mathrm{IFT}}\right] \hat{M}_{+}, \\
& \underline{D}_{K}=-\underline{\tilde{\zeta}}^{\mu} \cdot\left[\underline{\Delta}_{R} \cdot \underline{C}_{2}^{T T}-\underline{C}_{2}^{T T} \cdot \underline{\Delta}_{A}\right] \cdot \underline{\tilde{\zeta}}^{\nu}+\underline{\tilde{\zeta}}^{\mu} \cdot\left[\underline{\Delta}_{R} \cdot \underline{C}_{3}^{T T^{\prime}}-\underline{C}_{3}^{T T^{\prime}} \cdot \underline{\Delta}_{A}\right] \cdot \underline{E}_{\perp}^{\nu} \\
& -\underline{E}_{\perp}^{\mu} \cdot\left[\underline{\Delta}_{R} \cdot \underline{C}_{3}^{T^{\prime} T}-\underline{C}_{3}^{T^{\prime} T} \cdot \underline{\Delta}_{A}\right] \cdot \underline{\tilde{\zeta}}^{\nu} .
\end{aligned}
$$

$\hat{D}$ in Eq. (3.10) is the leading part of the DEX of $\hat{D}(P, X)(\stackrel{\mathrm{WT}}{\longleftarrow} \underline{\hat{D}}(x, y))$. Calculation within the gradient approximation yields

$$
\begin{aligned}
\left(\underline{\hat{D}}^{-1}\right)^{\mu \nu} \cdot \underline{\hat{D}}_{\nu}^{\rho}= & g^{\mu \rho} \\
\left(\underline{\hat{D}}^{-1}\right)^{\mu \nu}(x, y)= & \left(g^{\mu \nu} \partial^{2}-\partial^{\mu} \partial^{\nu}+\frac{1}{\lambda} \tilde{\partial}^{\mu} \tilde{\partial}^{\nu}\right)_{\mathrm{IFT}} \hat{\tau}_{3}-\underline{\underline{L}}_{c}^{\mu \nu}(x, y) \\
= & \hat{\tau}_{3}\left[\mathcal{P}_{T}^{\mu \nu}(i \partial) \partial^{2}+\mathcal{P}_{L}^{\mu \nu}(i \partial) \tilde{\partial}^{2}-\partial_{0}\left(\tilde{\partial}^{\mu} n^{\nu}+n^{\mu} \tilde{\partial}^{\nu}\right)+\mathcal{P}_{G}^{\mu \nu}(i \partial)\left(\frac{\tilde{\partial}^{2}}{\lambda}+\partial_{0}^{2}\right)\right]_{\mathrm{IFT}} \\
& -\hat{\underline{L}}_{c}^{\mu \nu}(x, y), \\
\hat{L}_{c}^{\mu \nu}= & L_{c}^{\mu \nu} \hat{M}_{-} \\
= & 2 i \hat{M}_{-}\left[(P \cdot \partial \tilde{f}) \mathcal{P}_{T}^{\mu \nu}+\tilde{P} \cdot \tilde{\partial} \tilde{f} n^{\mu} n^{\nu}\right. \\
& +\left\{\frac{1}{\tilde{P}^{2}}\left(\frac{p_{0}^{2}}{\tilde{P}^{2}}+\frac{2}{\lambda}\right) \tilde{P} \cdot \tilde{\partial} \tilde{f}+\frac{p_{0}}{\tilde{P}^{2}} \partial_{0} \tilde{f}\right\} \tilde{P}^{\mu} \tilde{P}^{\nu} \\
& -\left\{\frac{p_{0}}{\tilde{P}^{2}} \tilde{P} \cdot \tilde{\partial} \tilde{f}+\frac{1}{2} \partial_{0} \tilde{f}\right\}\left(n^{\mu} \tilde{P}^{\nu}+\tilde{P}^{\mu} n^{\nu}\right)+P \cdot \partial C_{2}^{T T}(P, X) \tilde{\zeta}^{\mu} \tilde{\zeta}^{\nu} \\
& \left.-P \cdot \partial C_{3}^{T T^{\prime}}(P, X) \tilde{\zeta}^{\mu} E_{\perp}^{\nu}+P \cdot \partial C_{3}^{T^{\prime} T}(P, X) E_{\perp}^{\mu} \tilde{\zeta}^{\nu}\right] .
\end{aligned}
$$

Here $P \cdot \partial \tilde{f}=P^{\mu} \partial \tilde{f}(P, X) / \partial X^{\mu}$, etc.

From Eq. (4.52), we see that the free action of the theory is 


$$
\begin{gathered}
\mathcal{A}_{0}=\frac{1}{2} \int d^{4} x d^{4} y{ }^{\mathrm{t}} \hat{A}^{\mu}(x)\left(\hat{D}^{-1}(x, y)\right)_{\mu \nu} \hat{A}^{\nu}(y), \\
{ }^{\mathrm{t}} \hat{A}^{\mu}=\left(\hat{A}_{1}^{\mu}, \hat{A}_{2}^{\mu}\right)
\end{gathered}
$$

where the color index is suppressed. Eq. (4.55) with Eq. (4.53) tells us that there emerges a counteraction,

$$
\mathcal{A}_{c}=\frac{1}{2} \int d^{4} x d^{4} y{ }^{\mathrm{t}} \hat{A}^{\mu}(x) \underline{\hat{L}}_{c}^{\mu \nu}(x, y) \hat{A}^{\nu}
$$

which yields a (two-point) vertex factor

$$
i \underline{\hat{L}}_{c}^{\mu \nu}(x, y) \rightarrow i L_{c}^{\mu \nu}(P, X) \hat{M}_{-} .
$$

\section{Dyson equation}

As in Sec. III, we use the $(4 \times 4)$ matrix notation in Minkowski space. The self-energypart $(\underline{\hat{\mathbf{M}}}(x, y))$ resummed propagator $\underline{\hat{\mathbf{G}}}(x, y)$ obeys

$$
\begin{aligned}
\underline{\hat{\mathbf{G}}}= & \underline{\hat{\mathbf{D}}}-\underline{\hat{\mathbf{D}}} \cdot \underline{\hat{\mathbf{I}}} \cdot \underline{\hat{\mathbf{G}}}, \\
\underline{\hat{\mathbf{D}}}= & \left(\begin{array}{cc}
\underline{\mathbf{D}}_{R} & 0 \\
\underline{\mathbf{D}}_{R}-\underline{\mathbf{D}}_{A} & -\underline{\mathbf{D}}_{A}
\end{array}\right) \\
& +\left[\underline{\mathbf{D}}_{R} \cdot \underline{\tilde{f}}-\underline{\tilde{f}} \cdot \underline{\mathbf{D}}_{A}+\underline{\mathbf{D}}_{K}\right] \hat{M}_{+} .
\end{aligned}
$$

For $\underline{\hat{\mathbf{G}}}$ and $\underline{\boldsymbol{\Pi}}$, we have (cf. Eqs. (3.27) -(3.31) and (3.20) - (3.24).)

$$
\begin{aligned}
& \underline{\hat{\mathbf{G}}}=\left(\begin{array}{cc}
\underline{\mathbf{G}}_{R} & 0 \\
\underline{\mathbf{G}}_{R}-\underline{\mathbf{G}}_{A} & -\underline{\mathbf{G}}_{A}
\end{array}\right) \\
& +\left[\underline{\mathbf{G}}_{R} \cdot \underline{\tilde{f}}-\underline{\tilde{f}} \cdot \underline{\mathbf{G}}_{A}+\underline{\mathbf{G}}_{K}\right] \hat{M}_{+}, \\
& \underline{\hat{\Pi}}=\left(\begin{array}{cc}
\underline{\Pi}_{R} & 0 \\
-\underline{\Pi}_{R}+\underline{\Pi}_{A} & -\underline{\Pi}_{A}
\end{array}\right) \\
& +\left[\underline{\Pi}_{R} \cdot \underline{\tilde{f}}-\underline{\tilde{f}} \cdot \underline{\Pi}_{A}-\underline{\Pi}_{K}\right] \hat{M}_{-}, \\
& \underline{\mathbf{G}}_{K}=-\underline{\mathbf{G}}_{R} \cdot \underline{\tilde{f}}+\underline{\tilde{f}} \cdot \underline{\mathbf{G}}_{A}+\underline{\mathbf{G}}_{12}, \\
& \underline{\Pi}_{K}=\underline{\Pi}_{R} \cdot \underline{\tilde{f}}-\underline{\tilde{f}} \cdot \underline{\Pi}_{A}+\underline{\Pi}_{12} \text {. }
\end{aligned}
$$


Eq. (4.56) may be solved to give

$$
\begin{aligned}
\underline{\mathbf{G}}_{R(A)}= & {\left[\underline{\mathbf{D}}_{R(A)}^{-1}+\underline{\mathbf{I}}_{R(A)}\right]^{-1}, } \\
\underline{\mathbf{G}}_{K}= & \underline{\mathbf{G}}_{K}^{[1]}+\underline{\mathbf{G}}_{K}^{[2]}+\underline{\mathbf{G}}_{K}^{[3]}, \\
\underline{\mathbf{G}}_{K}^{[1]}= & \underline{\mathbf{G}}_{R} \cdot \underline{\mathbf{\Pi}}_{K} \cdot \underline{\mathbf{G}}_{A}, \\
\underline{\mathbf{G}}_{K}^{[2]}= & \underline{\mathbf{G}}_{R} \cdot\left[\underline{\mathbf{I}}_{R} \cdot \underline{\mathbf{C}}-\underline{\mathbf{C}} \cdot \underline{\mathbf{I}}_{A}\right] \cdot \underline{\mathbf{G}}_{A} \\
\equiv & -\underline{\mathbf{G}}_{R} \cdot \underline{\tilde{\mathbf{H}}} \cdot \underline{\mathbf{G}}_{A}, \\
\underline{\mathbf{G}}_{K}^{[3]}= & \underline{\mathbf{G}}_{R} \cdot \underline{\mathbf{C}}-\underline{\mathbf{C}} \cdot \underline{\mathbf{G}}_{A}, \\
\underline{C}^{\mu \nu}= & \underline{\tilde{\zeta}}^{\mu} \cdot \underline{C}_{2}^{T T} \cdot \underline{\tilde{\zeta}}^{\nu}-\underline{\tilde{\zeta}}^{\mu} \cdot \underline{C}_{3}^{T T^{\prime}} \cdot \underline{E}_{\perp}^{\nu} \\
& +\underline{E}_{\perp}^{\mu} \cdot \underline{C}_{3}^{T^{\prime} T} \cdot \underline{\tilde{\zeta}}^{\nu} .
\end{aligned}
$$

The form for the leading part of the DEX of $\hat{G}(X, P)$, an Wigner transform of $\underline{G}(x, y)$, is the $\hat{G}$ that is deduced in Sec. III.

\section{Gradient piece of the self-energy-part resummed propagator}

$$
\text { Form for } G_{R(A)}
$$

We divide the Wigner transform of $\underline{\Pi}_{R(A)} \cdot \underline{\mathbf{G}}_{R(A)}$ (cf. Eq. (4.58)) into two pieces (cf. Eq. (4.51)),

$$
\begin{aligned}
\underline{\Pi}_{R(A)} \cdot \underline{\mathbf{G}}_{R(A)} \stackrel{\mathrm{WT}}{\longrightarrow} & \boldsymbol{\Pi}_{R(A)}(P, X) \mathbf{G}_{R(A)}(P, X)+\left(\boldsymbol{\Pi}_{R(A)} \mathbf{G}_{R(A)}\right)^{(1)} \\
\left(\boldsymbol{\Pi}_{R(A)} \mathbf{G}_{R(A)}\right)^{(1) \mu \nu}= & \frac{i}{2} \sum_{U, V, V^{\prime}=T, L, G} \sum_{j=1}^{J_{U V}} \sum_{j^{\prime}=1}^{J_{V V^{\prime}}}\left[\frac{\partial \mathcal{R}_{L j}^{U V}}{\partial P_{\mu}} \frac{\partial \tilde{\Pi}_{R(A) j}^{U V}}{\partial X^{\mu}} \mathcal{R}_{R j}^{U V} \mathcal{R}_{L j^{\prime}}^{V V^{\prime}} \tilde{G}_{R(A) j^{\prime}}^{V V^{\prime}} \mathcal{R}_{R j^{\prime}}^{V V^{\prime}}\right. \\
& -\mathcal{R}_{L j}^{U V} \frac{\partial \tilde{\Pi}_{R(A) j}^{U V}}{\partial X^{\mu}} \frac{\partial \mathcal{R}_{R j}^{U V} \mathcal{R}_{L j^{\prime}}^{V V^{\prime}} \tilde{G}_{R(A) j^{\prime}}^{V V^{\prime}} \mathcal{R}_{R j^{\prime}}^{V V^{\prime}}}{\partial P_{\mu}} \\
& +\frac{\partial \mathcal{R}_{L j}^{U V} \tilde{\Pi}_{R(A) j}^{U V} \mathcal{R}_{R j}^{U V} \mathcal{R}_{L j^{\prime}}^{V V^{\prime}}}{\partial P_{\mu}} \frac{\partial \tilde{G}_{R(A) j^{\prime}}^{V V^{\prime}}}{\partial X^{\mu}} \mathcal{R}_{R j^{\prime}}^{V V^{\prime}} \\
& -\mathcal{R}_{L j}^{U V} \tilde{\Pi}_{R(A) j}^{U V} \mathcal{R}_{R j}^{U V} \mathcal{R}_{L j^{\prime}}^{V V^{\prime}} \frac{\left.\partial \tilde{G}_{R(A) j^{\prime}}^{V V V^{\prime}} \frac{\partial \mathcal{R}_{R j^{\prime}}^{V V^{\prime}}}{\partial P_{\mu}}\right]^{\mu \nu}}{\partial X^{\mu}}
\end{aligned}
$$


Here, $\boldsymbol{\Pi}_{R(A)}(P, X)$ and $\mathbf{G}_{R(A)}(P, X)$ are, in respective order, $\boldsymbol{\Pi}_{R(A)}(P)$ and $\mathbf{G}_{R(A)}(P)$ in Sec III. Using Eq. (4.62) in Eq. (4.58), we obtain the solution for $\mathbf{G}_{R(A)}\left(=\mathbf{G}_{R(A)}^{(0)}+\mathbf{G}_{R(A)}^{(1)}\right)$. The form for the leading part $\mathbf{G}_{R(A)}^{(0)}$ is given in Sec. III. The gradient part is

$$
\begin{aligned}
\mathbf{G}_{R(A)}^{(1)}= & \mathbf{G}_{R(A)}^{(0)}\left[\left\{i g^{\mu \nu} P \cdot \partial-\frac{i}{2}\left(P^{\mu} \partial^{\nu}+\partial^{\mu} P^{\nu}\right)+\frac{i}{2} \lambda\left(\tilde{P}^{\mu} \tilde{\partial}^{\nu}+\tilde{\partial}^{\mu} \tilde{P}^{\nu}\right)\right\} \mathbf{G}_{R(A)}^{(0)}\right. \\
& \left.-\left(\boldsymbol{\Pi}_{R(A)} \mathbf{G}_{R(A)}^{(0)}\right)^{(1)}\right] .
\end{aligned}
$$

Form for $\Pi_{K}$, which is involved in $G_{K}^{[1]}$ in Eq. (4.59)

In the following, we restrict ourselves to the strict Coulomb gauge, $\lambda=0$, which is a physical gauge. Computation of Eq. (4.57) to the gradient approximation yields

$$
\begin{aligned}
\underline{\Pi}_{K}^{\mu \nu}= & -\underline{L}_{c}^{\mu \nu}+\sum_{j} \sum_{U V=T, L}\left[\underline{\mathcal{R}}_{L j}^{U V} \cdot\left\{\left(\tilde{f}\left[\left(\tilde{\Pi}_{12}\right)_{j}^{U V}-\left(\tilde{\Pi}_{21}\right)_{j}^{U V}\right]\right)_{\mathrm{IWT}}+\left(\underline{\tilde{\Pi}}_{12}\right)_{j}^{U V}\right\} \cdot \underline{\mathcal{R}}_{R j}^{U V}\right]^{\mu \nu} \\
& +\underline{\Pi}_{K}^{[1] \mu \nu}+\underline{\Pi}_{K}^{[2] \mu \nu} .
\end{aligned}
$$

In $\underline{G}_{K}^{[1] \mu \nu}$, Eq. (4.59), $\underline{\Pi}_{K}^{\mu \nu}$, and then $\underline{L}_{c}^{\mu \nu}$ in Eq. (4.64), are sandwiched between $\underline{\mathbf{G}}_{R}$ and $\underline{\mathbf{G}}_{A}$. Then, in the case of $\lambda=0, \tilde{P}^{\mu} \tilde{P}^{\nu}$ and $\left(n^{\mu} \tilde{P}^{\nu}+\tilde{P}^{\mu} n^{\nu}\right)$ terms in $\Pi_{c}^{\mu \nu}$, Eq. (4.54), do not contribute to $\mathbf{G}_{K}^{[1]}$ (cf. Sec. III).

The Standard forms for the gradient terms, $\Pi_{K}^{[1] \mu \nu}$ and $\Pi_{K}^{[2] \mu \nu}$, and $\tilde{H}$ (Eq. (4.60)) are given in Appendix G.

\section{Generalized Boltzmann equation and its relatives}

Structure of the theory is fully discussed in Sec. IVA5, so that we restrict ourselves to giving a brief description of the physical- $N$ scheme only.

Same reasoning as in Subsec. IVA applies here: $\mathbf{G}_{K}^{[1]}$ and $\mathbf{G}_{K}^{[2]}$, Eqs. (4.59) and (4.60), bring about disaster. This disaster would be overcome if the condition

$$
\Pi_{K}-\tilde{\mathbf{H}}=0
$$


could be imposed. This is, however, not possible. Eq. (4.65) may be imposed for the $\mathcal{P}_{T}^{\mu \nu}$, $n^{\mu} n^{\nu}, \tilde{\zeta}^{\mu} \tilde{\zeta}^{\nu}$, and $\tilde{\zeta}^{\mu} E_{\perp}^{\nu}$ components, which read, in respective order,

$$
\begin{aligned}
2 P \cdot \partial \tilde{f}= & -i\left[(1+\tilde{f})\left(\Pi_{12}\right)_{1}^{T T}-\tilde{f}\left(\Pi_{21}\right)_{1}^{T T}\right]-i \Pi_{K 1}^{T T}-2 \operatorname{Im}\left(\tilde{\zeta}^{2} E_{\perp}^{2} C_{3}^{T T^{\prime}} \Pi_{R 3}^{T^{\prime} T}\right)+i H_{1}^{(1) T T}, \\
2 \tilde{P} \cdot \tilde{\partial} \tilde{f}= & -i\left[(1+\tilde{f})\left(\Pi_{12}\right)_{1}^{L L}-\tilde{f}\left(\Pi_{21}\right)_{1}^{L L}\right]-i \Pi_{K 1}^{L L}, \\
2 P \cdot \partial C_{2}^{T T}= & -i\left[(1+\tilde{f})\left(\Pi_{12}\right)_{2}^{T T}-\tilde{f}\left(\Pi_{21}\right)_{2}^{T T}\right]-i \Pi_{K 2}^{(1) T T} \\
& -2 I m\left[-C_{2}^{T T}\left(\Pi_{R 1}^{T T}+\tilde{\zeta}^{2} \Pi_{R 2}^{T T}\right)+E_{\perp}^{2} C_{3}^{T^{\prime} T}\left(\Pi_{R 3}^{T T^{\prime}}+\Pi_{A 2}^{T T^{\prime}}\right)\right]+i H_{2}^{(1) T T}, \\
2 P \cdot \partial C_{3}^{T T^{\prime}}= & -i\left[(1+\tilde{f})\left(\Pi_{12}\right)_{3}^{T T^{\prime}}-\tilde{f}\left(\Pi_{21}\right)_{3}^{T T^{\prime}}\right]-i \Pi_{K 3}^{(1) T T^{\prime}} \\
& +i\left[\tilde{\zeta}^{2} C_{2}^{T T} \Pi_{A 3}^{T T^{\prime}}+C_{3}^{T T^{\prime}}\left(\Pi_{A 1}^{T T}-\Pi_{R 1}^{T T}-\tilde{\zeta}^{2} \Pi_{R 2}^{T T}\right)\right]+i H_{3}^{(1) T T^{\prime}} .
\end{aligned}
$$

Proceeding as in [10], from Eqs. (4.66) and (4.67) on the energy shells, we obtain the generalized Boltzmann equations for the transverse and the longitudinal modes, respectively. (As a matter of fact, on the energy shells, the first term on the RHS of Eq. (4.66) (Eq. (4.67)) is proportional to the net production rates of the transverse (longitudinal) mode.) We do not reproduce them here. It should be remarked that, in the case of $L$ mode, Eq. (4.67), the "time-derivative term", $\partial_{0} \tilde{f}$, in the Boltzmann equation comes from $\Pi_{K 1}^{L L}$. More

precisely, the $\partial_{0} \tilde{f}$ term comes from $\Pi_{K}^{[1] \mu \nu}$ (with $U V=L L$ ), Eq. (G.1) in Appendix G, which is in $\Pi_{K 1}^{L L}$ in Eq. (4.67). Eq. (4.68) [(4.69)] determines spacetime evolution of $C_{2}^{T T}\left[C_{3}^{T T^{\prime}}\right]$ along $P$. An evolution equation for $C_{3}^{T^{\prime} T}$ is obtained from Eqs. (4.69) and (3.16).

One cannot impose Eq. (4.65) for the remaining $\tilde{\zeta}^{\mu} n^{\nu}, n^{\mu} \tilde{\zeta}^{\nu}, E_{\perp}^{\mu} n^{\nu}$, and $n^{\mu} E_{\perp}^{\nu}$ components. This is because, for these components, there are no counterpart of $\tilde{f}, C_{2}^{T T}, C_{3}^{T T^{\prime}}$, and $C_{3}^{T^{\prime} T}$. For equilibrium systems, these modes are absent. Then, one can expect that, for the quasiuniform systems near equilibrium, these modes do not yield disastrously large contributions.

\section{Ghost sector}

The self-energy-part $(\underline{\hat{\tilde{\Pi}}}(x, y))$ resummed propagator $\underline{\hat{\tilde{\mathbf{G}}}}(x, y)$ obeys 


$$
\underline{\hat{\tilde{\mathbf{G}}}}=\underline{\hat{\tilde{\mathbf{D}}}} \cdot \underline{\hat{\tilde{\Pi}}} \cdot \underline{\tilde{\tilde{\mathbf{G}}}} \cdot
$$

$\underline{\hat{\tilde{\mathbf{D}}}}$ is an inverse Fourier transform of $\hat{\tilde{\mathbf{D}}}$ in Eq. (3.17). As in Eq. (3.44), $\underline{\hat{\tilde{\mathbf{D}}}}, \underline{\hat{\tilde{\mathbf{I}}}}$, and $\underline{\hat{\tilde{\mathbf{G}}}}$ are diagonal $(2 \times 2)$-matrix functions, $\underline{\hat{\tilde{\mathbf{D}}}}=\underline{\tilde{\mathbf{D}}} \hat{\tau}_{3}=$, etc. Solving Eq. (4.70), we see that the gradient part of $\tilde{G}(P, X)$ vanishes and

$$
\hat{\tilde{G}}(P, X)=(\hat{\tilde{G}}(P, X))^{*}=\frac{1}{\tilde{P}^{2}-\tilde{\Pi}(P, X)} .
$$

\section{ACKNOWLEDGMENTS}

The author thanks the useful discussion at the Workshop on Thermal Field Theories and their Applications, held at the Yukawa Institute for Theoretical Physics, Kyoto, Japan, 8 10 August, 2002.

\section{APPENDIX A: RESUMMATION OF THE QUARK PROPAGATOR}

Here we derive Eqs. (2.39) - (2.42). Formally solving Eq. (2.33), we obtain

$$
\begin{aligned}
\mathbf{G}_{K} & =-\mathbf{G}_{R} \boldsymbol{\Sigma}_{K} \mathbf{G}_{A}+\mathbf{G}_{K}^{\prime}, \\
\mathbf{G}_{K}^{\prime} & =\mathbf{G}_{R} S_{R}^{-1} \mathbf{S}_{K}\left(1+\boldsymbol{\Sigma}_{A} \mathbf{G}_{A}\right),
\end{aligned}
$$

where use has been made of Eq. (2.32). Since $S_{R}^{-1} \mathbf{S}_{K} \propto\left(P^{2}-m^{2}\right) \delta\left(P^{2}-m^{2}\right)=0$, we have $\mathbf{G}_{K}^{\prime}=0$. This means that the piece $\mathbf{S}_{K}$ of the bare propagator disappears through resummation, which is unnatural.

A correct $\mathbf{G}_{K}^{\prime}$ is obtained by substituting Eq. (2.18) for $\mathbf{S}_{K}$ in Eq. (A.1) as follows:

$$
\begin{aligned}
\mathbf{G}_{K}^{\prime}= & \mathbf{G}_{R} S_{R}^{-1}\left(S_{R}-S_{A}\right) \gamma_{5} N^{\prime} \mathbf{C}(P)\left(1+\boldsymbol{\Sigma}_{A} \mathbf{G}_{A}\right) \\
= & {\left[\mathbf{G}_{R} S_{R}^{-1} S_{R} \gamma_{5} N^{\dagger}-\mathbf{G}_{R} S_{R}^{-1} \gamma_{5} N S_{A}\right] \mathbf{C}(P) } \\
& \times\left(1+\boldsymbol{\Sigma}_{A} \mathbf{G}_{A}\right) \\
= & \mathbf{G}_{R} \gamma_{5} N \mathbf{C}(P)\left(1+\boldsymbol{\Sigma}_{A} \mathbf{G}_{A}\right) \\
& -\left(1+\mathbf{G}_{R} \boldsymbol{\Sigma}_{R}\right) \gamma_{5} N \mathbf{C}(P) \mathbf{G}_{A},
\end{aligned}
$$


where use has been made of $\mathbf{G}_{R} S_{R}^{-1}=\left(\mathbf{1}+\mathbf{G}_{R} \boldsymbol{\Sigma}_{R}\right)$, which follows from Eq. (2.32). This is natural in the sense that

$$
\mathbf{G}_{K} \stackrel{\hat{\boldsymbol{\Sigma}} \rightarrow 0}{\longrightarrow} \mathbf{S}_{K}
$$

\section{APPENDIX B: "MULTIPLICATIONS" OF THE TWO STANDARD FORMS FOR THE QUARK PART}

We define "multiplications" of the functions of the type (2.5) as the products:

$$
(A \otimes B)^{\rho \sigma} \equiv A^{\rho \rho} B^{\rho \sigma}, \quad[A \otimes B]^{\rho \sigma} \equiv A^{\rho-\rho} B^{-\rho \sigma}
$$

Straightforward manipulation yields the SFs (cf. Eq. (2.5)) of $(A \otimes B)^{\rho \sigma}$,

$$
\begin{aligned}
(A \otimes B)_{1}^{\rho \pm \rho}= & A_{1}^{\rho \rho} B_{1}^{\rho \pm \rho} \pm P^{2} A_{2}^{\rho \rho} B_{2}^{\rho \pm \rho} \pm N^{2} A_{3}^{\rho \rho} B_{3}^{\rho \pm \rho} \\
& -P^{2} N^{2} A_{4}^{\rho \rho} B_{4}^{\rho \pm \rho}, \\
(A \otimes B)_{2}^{\rho \pm \rho}= & A_{1}^{\rho \rho} B_{2}^{\rho \pm \rho} \pm A_{2}^{\rho \rho} B_{1}^{\rho \pm \rho} \mp N^{2} A_{3}^{\rho \rho} B_{4}^{\rho \pm \rho} \\
& +N^{2} A_{4}^{\rho \rho} B_{3}^{\rho \pm \rho} \\
(A \otimes B)_{3}^{\rho \pm \rho}= & A_{1}^{\rho \rho} B_{3}^{\rho \pm \rho} \pm P^{2} A_{2}^{\rho \rho} B_{4}^{\rho \pm \rho} \pm A_{3}^{\rho \rho} B_{1}^{\rho \pm \rho} \\
& -P^{2} A_{4}^{\rho \rho} B_{2}^{\rho \pm \rho}, \\
(A \otimes B)_{4}^{\rho \pm \rho}= & A_{1}^{\rho \rho} B_{4}^{\rho \pm \rho} \pm A_{2}^{\rho \rho} B_{3}^{\rho \pm \rho} \mp A_{3}^{\rho \rho} B_{2}^{\rho \pm \rho} \\
& +A_{4}^{\rho \rho} B_{1}^{\rho \pm \rho} .
\end{aligned}
$$

$[A \otimes B]_{j}^{\rho-\rho}(j=1-4)$ is given by $(A \otimes B)_{j}^{\rho \rho}$ in Eq. (B.2) with $B_{j}^{-\rho-\rho}$ for $B_{j}^{\rho \rho}$, and $[A \otimes B]_{j}^{\rho \rho}$ is given by $(A \otimes B)_{j}^{\rho-\rho}$ in Eq. (B.2) with $B_{j}^{-\rho \rho}$ for $B_{j}^{\rho-\rho}$. 


\section{APPENDIX C: "MULTIPLICATION" OF THE TWO STANDARD FORMS FOR THE GLUON PART}

We define a "multiplication" of the functions, $A^{\mu \nu}$ and $B^{\mu \nu}$, of the type, (3.6) with (3.7), by $C^{\mu \nu}=A^{\mu \rho} B_{\rho}{ }^{\nu}$. Straightforward computation yields the SF for $C^{\mu \nu}$ :

$$
\begin{aligned}
& C_{1}^{T T}=A_{1}^{T T} B_{1}^{T T}+\tilde{P}^{2} \tilde{\zeta}^{4} n^{2} A_{3}^{T^{\prime} T} B_{3}^{T T^{\prime}}+\tilde{P}^{2} \tilde{\zeta}^{2} n^{4} A_{2}^{T L} B_{2}^{L T}-\tilde{P}^{4} \tilde{\zeta}^{2} n^{2} A_{1}^{T G} B_{1}^{G T} \\
& C_{2}^{T T}=A_{1}^{T T} B_{2}^{T T}+A_{2}^{T T} B_{1}^{T T}+\tilde{\zeta}^{2} A_{2}^{T T} B_{2}^{T T}+\tilde{P}^{2} \tilde{\zeta}^{2} n^{2} A_{3}^{T T^{\prime}} B_{3}^{T^{\prime} T} \\
& -\tilde{P}^{2} \tilde{\zeta}^{2} n^{2} A_{3}^{T^{\prime} T} B_{3}^{T T^{\prime}}+n^{2} A_{1}^{T L} B_{1}^{L T}-\tilde{P}^{2} n^{4} A_{2}^{T L} B_{2}^{L T}+\tilde{P}^{4} n^{2} A_{1}^{T G} B_{1}^{G T} \\
& -\tilde{P}^{2} A_{2}^{T G} B_{2}^{G T} \\
& C_{3}^{T T^{\prime}}=A_{1}^{T T} B_{3}^{T T^{\prime}}+\tilde{\zeta}^{2} A_{2}^{T T} B_{3}^{T T^{\prime}}+A_{3}^{T T^{\prime}} B_{1}^{T T}+n^{2} A_{1}^{T L} B_{2}^{L T}-\tilde{P}^{2} A_{2}^{T G} B_{1}^{G T}, \\
& C_{3}^{T^{\prime} T}=A_{1}^{T T} B_{3}^{T^{\prime} T}+A_{3}^{T^{\prime} T} B_{1}^{T T}+\tilde{\zeta}^{2} A_{3}^{T^{\prime} T} B_{2}^{T T}+n^{2} A_{2}^{T L} B_{1}^{L T}-\tilde{P}^{2} A_{1}^{T G} B_{2}^{G T}, \\
& C_{1}^{L L}=\tilde{\zeta}^{2} n^{2} A_{1}^{L T} B_{1}^{T L}+\tilde{P}^{2} \tilde{\zeta}^{2} n^{4} A_{2}^{L T} B_{2}^{T L}+A_{1}^{L L} B_{1}^{L L}-\tilde{P}^{2} n^{2} A_{1}^{L G} B_{1}^{G L}, \\
& C_{1}^{G G}=-\tilde{P}^{4} \tilde{\zeta}^{2} n^{2} A_{1}^{G T} B_{1}^{T G}-\tilde{P}^{2} \tilde{\zeta}^{2} A_{2}^{G T} B_{2}^{T G}-\tilde{P}^{2} n^{2} A_{1}^{G L} B_{1}^{L G}+A_{1}^{G G} B_{1}^{G G}, \\
& C_{1}^{T L}=A_{1}^{T T} B_{1}^{T L}+\tilde{\zeta}^{2} A_{2}^{T T} B_{1}^{T L}+\tilde{P}^{2} \tilde{\zeta}^{2} n^{2} A_{3}^{T T^{\prime}} B_{2}^{T L}+A_{1}^{T L} B_{1}^{L L}-P^{2} A_{2}^{T G} B_{1}^{G L}, \\
& C_{2}^{T L}=A_{1}^{T T} B_{2}^{T L}+\tilde{\zeta}^{2} A_{3}^{T^{\prime} T} B_{1}^{T L}+A_{2}^{T L} B_{1}^{L L}-\tilde{P}^{2} A_{1}^{T G} B_{1}^{G L}, \\
& C_{1}^{L T}=A_{1}^{L T} B_{1}^{T T}+\tilde{\zeta}^{2} A_{1}^{L T} B_{2}^{T T}+\tilde{P}^{2} \tilde{\zeta}^{2} n^{2} A_{2}^{L T} B_{3}^{T^{\prime} T}+A_{1}^{L L} B_{1}^{L T}-\tilde{P}^{2} A_{1}^{L G} B_{2}^{G T}, \\
& C_{2}^{L T}=\tilde{\zeta}^{2} A_{1}^{L T} B_{3}^{T T^{\prime}}+A_{2}^{L T} B_{1}^{T T}+A_{1}^{L L} B_{2}^{L T}-\tilde{P}^{2} A_{1}^{L G} B_{1}^{G T}, \\
& C_{1}^{T G}=A_{1}^{T T} B_{1}^{T G}+\tilde{\zeta}^{2} A_{3}^{T^{\prime} T} B_{2}^{T G}+n^{2} A_{2}^{T L} B_{1}^{L G}+A_{1}^{T G} B_{1}^{G G} \\
& C_{2}^{T G}=A_{1}^{T T} B_{2}^{T G}+\tilde{\zeta}^{2} A_{2}^{T T} B_{2}^{T G}+\tilde{P}^{2} \tilde{\zeta}^{2} n^{2} A_{3}^{T T^{\prime}} B_{1}^{T G}+n^{2} A_{1}^{T L} B_{1}^{L G}+A_{2}^{T G} B_{1}^{G G}, \\
& C_{1}^{G T}=A_{1}^{G T} B_{1}^{T T}+\tilde{\zeta}^{2} A_{2}^{G T} B_{3}^{T T^{\prime}}+n^{2} A_{1}^{G L} B_{2}^{L T}+A_{1}^{G G} B_{1}^{G T}, \\
& C_{2}^{G T}=\tilde{P}^{2} \tilde{\zeta}^{2} n^{2} A_{1}^{G T} B_{3}^{T T^{\prime}}+A_{2}^{G T} B_{1}^{T T}+\tilde{\zeta}^{2} A_{2}^{G T} B_{2}^{T T}+\tilde{n}^{2} A_{1}^{G L} B_{1}^{L T}, \\
& C_{1}^{L G}=\tilde{\zeta}^{2} A_{1}^{L T} B_{2}^{T G}+\tilde{P}^{2} \tilde{\zeta}^{2} n^{2} A_{2}^{L T} B_{1}^{T G}+A_{1}^{L L} B_{1}^{L G}+A_{1}^{L G} B_{1}^{G G}, \\
& C_{1}^{G L}=\tilde{P}^{2} \tilde{\zeta}^{2} n^{2} A_{1}^{G T} B_{2}^{T L}+\tilde{\zeta}^{2} A_{2}^{G T} B_{1}^{T L}+A_{1}^{G L} B_{1}^{L L}+A_{1}^{G G} B_{1}^{G L} .
\end{aligned}
$$




\section{APPENDIX D: GLUON PROPAGATOR IN A COVARIANT GAUGE}

Here we present a "translation table" to get the expressions for the gluon propagator in a covariant-gauge from the Coulomb-gauge counterparts given in Sec. III.

An orthogonal basis in Minkowski space is given by Eq. (3.1) with the replacements ${ }^{4}$,

$$
\begin{aligned}
&\left(\tilde{P}^{\mu}, \tilde{\zeta}^{\mu}, n^{\mu}, E_{\perp}^{\mu}\right) \Rightarrow\left(P^{\mu}, \tilde{\zeta}^{\mu}, \tilde{n}^{\mu}, E_{\perp}^{\mu}\right) \\
& \tilde{n}^{\mu} \equiv n^{\mu}-\frac{n \cdot P}{P^{2}} P^{\mu}=n^{\mu}-\frac{p_{0}}{P^{2}} P^{\mu} \\
&\left(\tilde{n}^{2}=-\frac{\vec{p}^{2}}{P^{2}}\right)
\end{aligned}
$$

Then, among the projection operators, Eqs. (3.2) - (3.4), $\mathcal{P}_{L}^{\mu \nu}$ and $\mathcal{P}_{G}^{\mu \nu}$ are replaced as

$$
\begin{gathered}
\mathcal{P}_{L}^{\mu \nu}(P)=\frac{n^{\mu} n^{\nu}}{n^{2}} \Rightarrow \mathcal{P}_{L}^{\mu \nu}(P)=\frac{\tilde{n}^{\mu} \tilde{n}^{\nu}}{\tilde{n}^{2}} \\
\mathcal{P}_{G}^{\mu \nu}(P)=\frac{\tilde{P}^{\mu} \tilde{P}^{\nu}}{\tilde{P}^{2}} \Rightarrow \mathcal{P}_{G}^{\mu \nu}(P)=\frac{P^{\mu} P^{\nu}}{P^{2}}
\end{gathered}
$$

$\mathcal{P}_{T}$ is the same as in Eq. (3.2).

Eq. (3.9) is replaced with

$$
\left(\hat{D}^{-1}(P)\right)^{\mu \nu}=-P^{2}\left[\mathcal{P}_{T}^{\mu \nu}+\mathcal{P}_{L}^{\mu \nu}+\frac{1}{\lambda} \mathcal{P}_{G}^{\mu \nu}\right] \hat{\tau}_{3},
$$

which is already in SF.

The SF-elements of $\mathbf{D}_{K}$ in Eq. (3.13) are replaced by

$$
\begin{aligned}
& D_{K 2}^{T T}(P)=2 \pi i C_{2}^{T T}(P) \epsilon\left(p_{0}\right) \delta\left(P^{2}\right), \\
& D_{K 3}^{T T^{\prime}}(P)=2 \pi i C_{3}^{T T^{\prime}}(P) \epsilon\left(p_{0}\right) \delta\left(P^{2}\right), \\
& D_{K 1}^{T L}(P)=2 \pi i C_{1}^{T L}(P) \epsilon\left(p_{0}\right) \delta\left(P^{2}\right), \\
& D_{K 2}^{T L}(P)=2 \pi i C_{2}^{T L}(P) \epsilon\left(p_{0}\right) \delta\left(P^{2}\right), \\
& D_{K j}^{U V}(P)=0 \quad \text { (otherwise). }
\end{aligned}
$$

\footnotetext{
${ }^{4}$ It should be noted that $E_{\perp}^{\mu}=\epsilon^{\mu \nu \rho \sigma} P_{\nu} \tilde{\zeta}_{\rho} \tilde{n}_{\sigma}=\epsilon^{\mu \nu \rho \sigma} \tilde{P}_{\nu} \tilde{\zeta}_{\rho} n_{\sigma}$
} 
In obtaining these, we have used the fact that $\left(\hat{D}_{U G}\right)^{\mu \nu}=\left(\hat{D}_{G U}\right)^{\mu \nu}=0(U=T, L)$, which is verified from the "bare counterparts" of Eq. (D.2), below. $D_{R}^{\mu \nu}$ in Eq. (3.15) is replaced with

$$
D_{R}^{\mu \nu}=\left(D_{A}^{\mu \nu}\right)^{*}=-\Delta_{R} \mathcal{P}_{T}^{\mu \nu}+\frac{d \Delta_{R}}{d P^{2}} P^{2}\left(\mathcal{P}_{L}^{\mu \nu}+\lambda \mathcal{P}_{G}^{\mu \nu}\right)
$$

Eq. (3.16) is replaced with

$$
\begin{array}{ll}
\left(C_{2}^{T T}\right)^{*}=C_{2}^{T T}, \quad & \left(C_{3}^{T T^{\prime}}\right)^{*}=-C_{3}^{T^{\prime} T}, \\
\left(C_{1}^{T L}\right)^{*}=C_{1}^{L T}, & \left(C_{2}^{T L}\right)^{*}=-C_{2}^{L T} .
\end{array}
$$

Eq. (3.17) is replaced by

$$
\tilde{\hat{D}}(P)=\left(\begin{array}{cc}
\Delta_{R} & 0 \\
\Delta_{R}-\Delta_{A} & -\Delta_{A}
\end{array}\right)+\tilde{f}\left(\Delta_{R}-\Delta_{A}\right) \hat{M}_{+} .
$$

Introduction of $\boldsymbol{\Pi}_{R}^{\prime}$, Eq. (3.39), is not necessary, $\mathbf{D}_{0}^{-1}=\mathbf{D}^{-1}$ and $\boldsymbol{\Pi}_{R}^{\prime}=\boldsymbol{\Pi}_{R}$, and, Eqs. (3.39) - (3.41) are deleted.

Description after Eq. (3.43) is replaced with the following one: Solving Eq. (3.43), we obtain

$$
\hat{\tilde{G}}=\left(\begin{array}{cc}
\tilde{G}_{R} & 0 \\
\tilde{G}_{R}-\tilde{G}_{A} & -\tilde{G}_{A}
\end{array}\right)+\left[\tilde{f}\left(\tilde{G}_{R}-\tilde{G}_{A}\right)+\tilde{G}_{K}\right] \hat{M}_{+},
$$

where

$$
\begin{aligned}
\tilde{G}_{R}(P) & =\tilde{G}_{A}^{*}(P)=\frac{1}{P^{2}-\tilde{\Pi}_{R}(P)}, \\
\tilde{G}_{K}(P) & =-\tilde{G}_{R}(P) \tilde{\Pi}_{K}(P) \tilde{G}_{A}(P), \\
\tilde{\Pi}_{R} & =\tilde{\Pi}_{A}^{*}=\tilde{\Pi}_{11}+\tilde{\Pi}_{12}=-\tilde{\Pi}_{22}-\tilde{\Pi}_{21}, \\
\tilde{\Pi}_{K} & =(1+\tilde{f}) \tilde{\Pi}_{11}-\tilde{f} \tilde{\Pi}_{21} .
\end{aligned}
$$

Eq. (3.46) is replaced with

$$
\hat{G}_{\mu \nu} P^{\nu}=\lambda\left[\hat{\tau}_{3} \hat{\tilde{\Pi}}_{\mu}-P_{\mu}\right] \hat{\tilde{G}}
$$


Eq. (3.47) is deleted.

Eqs. (3.48) - (3.52) are replaced by

$$
\begin{aligned}
& G_{R 1}^{G G}(P)=\lambda \frac{1}{P^{2}+i p_{0} 0^{+}}\left[\tilde{\Pi}(P)-P^{2}\right] \tilde{G}_{R}(P)=-\lambda \frac{1}{P^{2}+i p_{0} 0^{+}} \\
& G_{R 1}^{T G}(P)=\lambda \frac{1}{\left(P^{2}+i p_{0} 0^{+}\right) E_{\perp}^{2}}\left(E_{\perp}^{\mu} \tilde{\Pi}_{R \mu}(P)\right) \tilde{G}_{R}(P) \\
& G_{R 2}^{T G}(P)=\lambda \frac{1}{\left(P^{2}+i p_{0} 0^{+}\right) \tilde{\zeta}^{2}}\left(\tilde{\zeta}^{\mu} \tilde{\Pi}_{R \mu}(P)\right) \tilde{G}_{R}(P) \\
& G_{R 1}^{L G}(P)=\lambda \frac{1}{\left(P^{2}+i p_{0} 0^{+}\right) \tilde{n}^{2}}\left(\tilde{n}^{\mu} \tilde{\Pi}_{R \mu}(P)\right) \tilde{G}_{R}(P) \\
& G_{K 1}^{G G}(P)=-\lambda\left[\frac{P^{2}\left(P^{2}-\tilde{\Pi}_{R}\right) \tilde{G}_{K}}{\left(P^{2}+i 0^{+}\right)\left(P^{2}-i 0^{+}\right)}+\frac{\tilde{\Pi}_{K} \tilde{G}_{A}}{P^{2}-i p_{0} 0^{+}}\right]=0 \\
& G_{K 1}^{T G}(P)=\lambda \frac{1}{E_{\perp}^{2}}\left[\frac{E_{\perp}^{\mu} \tilde{\Pi}_{R \mu} P^{2} \tilde{G}_{K}}{\left(P^{2}+i 0^{+}\right)\left(P^{2}-i 0^{+}\right)}-\frac{E_{\perp}^{\mu} \tilde{\Pi}_{K \mu} \tilde{G}_{A}}{P^{2}-i p_{0} 0^{+}}\right] \\
& G_{K 2}^{T G}(P)=\lambda \frac{1}{\tilde{\zeta}^{2}}\left[\frac{\tilde{\zeta}^{\mu} \tilde{\Pi}_{R \mu} P^{2} \tilde{G}_{K}}{\left(P^{2}+i 0^{+}\right)\left(P^{2}-i 0^{+}\right)}-\frac{\tilde{\zeta}^{\mu} \tilde{\Pi}_{K \mu} \tilde{G}_{A}}{P^{2}-i p_{0} 0^{+}}\right] \\
& G_{K 1}^{L G}(P)=\lambda \frac{1}{\tilde{n}^{2}}\left[\frac{\tilde{n}^{\mu} \tilde{\Pi}_{R \mu} P^{2} \tilde{G}_{K}}{\left(P^{2}+i 0^{+}\right)\left(P^{2}-i 0^{+}\right)}-\frac{\tilde{n}^{\mu} \tilde{\Pi}_{K \mu} \tilde{G}_{A}}{P^{2}-i p_{0} 0^{+}}\right]
\end{aligned}
$$

In Eqs. (3.53) - (3.54), the replacements (D.1) and $\left(\Pi_{j}^{\prime}\right)_{U V}$ 's $\rightarrow\left(\Pi_{j}\right)_{U V}$ 's are made, and, in the formulae in Appendix C, the replacement (D.1) is made.

\section{APPENDIX E: STANDARD FORMS FOR THE QUANTITIES IN SEC. IV A}

$$
\text { Standard form for } \Sigma_{K}^{[1]}
$$

From Eq. (4.27), we obtain, after some algebra,

$$
\begin{aligned}
\Sigma_{K}^{[1]}= & -\frac{i}{2} \sum_{\rho= \pm} \mathcal{P}_{\rho}\left[2\left\{f_{\rho}, \quad R e \Sigma_{R}^{\rho \rho}\right\}^{\prime} \cdot \mathcal{P}_{\rho}+\left\{f_{-\rho}, \Sigma_{R}^{\rho-\rho}\right\}^{\prime} \cdot \mathcal{P}_{-\rho}+\left\{f_{\rho}, \Sigma_{A}^{\rho-\rho}\right\}^{\prime} \mathcal{P}_{-\rho}\right. \\
& +2 \frac{\partial f_{\rho}}{\partial X^{\alpha}} \operatorname{Re}\left\{\frac{\not P}{P^{2}}\left(P^{\alpha} \Sigma_{R 2}^{\rho \rho}-N^{\alpha} \Sigma_{R 3}^{\rho \rho}-\rho \epsilon\left(p_{0}\right) e_{\perp}^{\alpha} \Sigma_{R 4}^{\rho \rho}\right)\right. \\
& +\frac{\not X}{N^{2}}\left(N^{\alpha} \Sigma_{R 2}^{\rho \rho}+\frac{1}{2} \frac{\partial N^{2}}{\partial P_{\alpha}} \Sigma_{R 3}^{\rho \rho}-\rho \epsilon\left(p_{0}\right) e_{\perp}^{\mu} \frac{\partial N^{\mu}}{\partial P_{\alpha}} \Sigma_{R 4}^{\rho \rho}\right) \\
& \left.+\frac{\not P X}{P^{2} N^{2}} \rho \epsilon\left(p_{0}\right)\left(-e_{\perp}^{\alpha} \Sigma_{R 2}^{\rho \rho}-e_{\perp}^{\mu} \frac{\partial N_{\mu}}{\partial P_{\alpha}} \Sigma_{R 3}^{\rho \rho}+\rho \epsilon\left(p_{0}\right) N^{2} \hat{P}^{\alpha} \Sigma_{R 4}^{\rho \rho}\right)\right\}
\end{aligned}
$$




$$
\begin{aligned}
& +\gamma_{5}\left\{\frac{\not p}{P^{2}}\left(P^{\alpha}\left(\Lambda_{2}^{\rho-\rho}\right)_{\alpha}-N^{\alpha}\left(\Lambda_{3}^{\rho-\rho}\right)_{\alpha}+\rho \epsilon\left(p_{0}\right) e_{\perp}^{\alpha}\left(\Lambda_{4}^{\rho-\rho}\right)_{\alpha}\right)\right. \\
& +\frac{\not \mathcal{X}}{N^{2}}\left(N^{\alpha}\left(\Lambda_{2}^{\rho-\rho}\right)_{\alpha}+\frac{1}{2} \frac{\partial N^{2}}{\partial P_{\alpha}}\left(\Lambda_{3}^{\rho-\rho}\right)_{\alpha}+\rho \epsilon\left(p_{0}\right) e_{\perp}^{\mu} \frac{\partial N^{\mu}}{\partial P_{\alpha}}\left(\Lambda_{4}^{\rho-\rho}\right)_{\alpha}\right) \\
& \left.\left.+\frac{\not P X}{P^{2} N^{2}} \rho \epsilon\left(p_{0}\right)\left(e_{\perp}^{\alpha}\left(\Lambda_{2}^{\rho-\rho}\right)_{\alpha}+e_{\perp}^{\mu} \frac{\partial N_{\mu}}{\partial P_{\alpha}}\left(\Lambda_{3}^{\rho-\rho}\right)_{\alpha}+\rho \epsilon\left(p_{0}\right) N^{2} \hat{P}^{\alpha}\left(\Lambda_{4}^{\rho-\rho}\right)_{\alpha}\right)\right\}\right]
\end{aligned}
$$

where

$$
\begin{aligned}
& \hat{P}^{\alpha} \equiv P^{\alpha}+\frac{P^{2}}{2 N^{2}} \frac{\partial N^{2}}{\partial P_{\alpha}}, \\
& \left(\Lambda_{j}^{\rho-\rho}\right)_{\alpha} \equiv \Sigma_{R j}^{\rho-\rho} \frac{\partial f^{-\rho}}{\partial X^{\alpha}}+\Sigma_{A j}^{\rho-\rho} \frac{\partial f^{\rho}}{\partial X^{\alpha}} \quad(j=2,3,4), \\
& \left\{f_{-\rho}, \quad \Sigma_{R}^{\rho-\rho}\right\}^{\prime} \equiv \gamma_{5}\left[\left\{f_{-\rho}, \quad \Sigma_{R 1}^{\rho-\rho}\right\}+\left\{f_{-\rho}, \Sigma_{R 2}^{\rho-\rho}\right\} \not P\right. \\
& \left.+\left\{\begin{array}{ll}
f_{-\rho}, & \Sigma_{R 3}^{\rho-\rho}
\end{array}\right\} \mathbb{N}+\left\{f_{-\rho}, \quad \Sigma_{R 4}^{\rho-\rho}\right\} \not P D T\right],
\end{aligned}
$$

etc. Here, $\{\ldots, \ldots\}$ is as in Eq. (4.4).

Standard form for $\underline{H}$ in $G_{K}^{[2]}$ in Eq. (4.19)

Straightforward manipulation of Eq. (4.19) yields

$$
\begin{aligned}
\underline{H}= & \sum_{\rho, \sigma= \pm} \underline{\mathcal{P}}_{\rho} \cdot\left[H_{l}^{\rho \sigma}\right]_{\mathrm{IWT}} \cdot \underline{\mathcal{P}}_{\sigma}+\underline{H}^{(1)} \\
H^{(1)}= & \frac{i}{2} \sum_{\rho, \sigma= \pm} \mathcal{P}_{\rho} \gamma_{5}\left(\frac{\partial \mathcal{X}}{\partial P_{\alpha}} \frac{\partial C_{\rho-\rho} \Sigma_{A}^{-\rho \sigma}}{\partial X^{\alpha}}-\mathcal{X}\left\{C_{\rho-\rho}, \Sigma_{A}^{-\rho \sigma}\right\}\right) \mathcal{P}_{\sigma} \\
& +\frac{i}{2} \sum_{\rho, \sigma= \pm} \mathcal{P}_{\rho}\left(-\frac{\partial \Sigma_{R}^{\rho \sigma} C_{\sigma-\sigma}}{\partial X^{\alpha}} \frac{\partial \mathcal{X}}{\partial P_{\alpha}}+\left\{C_{\sigma-\sigma}, \Sigma_{R}^{\rho \sigma}\right\} X^{\prime}\right) \gamma_{5} \mathcal{P}_{-\sigma}
\end{aligned}
$$

where $H_{l}^{\rho \sigma}$ is as in Eq. (2.51). The SF for each term on the RHS of Eq. (E.1) reads

$$
\begin{aligned}
\mathcal{P}_{\rho} \gamma_{5} \frac{\partial X}{\partial P_{\alpha}} \frac{\partial C_{\rho-\rho} \Sigma_{A}^{-\rho \rho}}{\partial X^{\alpha}} \mathcal{P}_{\rho}= & \mathcal{P}_{\rho} \frac{\partial N_{\mu}}{\partial P_{\alpha}} \frac{\partial}{\partial X^{\alpha}}\left[C _ { \rho - \rho } \left\{-P^{\mu} \Sigma_{A 2}^{-\rho \rho}-N^{\mu} \Sigma_{A 3}^{-\rho \rho}-\rho \epsilon\left(p_{0}\right) e_{\perp}^{\mu} \Sigma_{A 4}^{-\rho \rho}\right.\right. \\
& +\frac{\not P}{P^{2}}\left(-P^{\mu} \Sigma_{A 1}^{-\rho \rho}+P^{2} N^{\mu} \Sigma_{A 4}^{-\rho \rho}+\rho \epsilon\left(p_{0}\right) e_{\perp}^{\mu} \Sigma_{A 3}^{-\rho \rho}\right) \\
& +\frac{\not \mathcal{X}}{N^{2}}\left(-N^{2} P^{\mu} \Sigma_{A 4}^{-\rho \rho}-N^{\mu} \Sigma_{A 1}^{-\rho \rho}-\rho \epsilon\left(p_{0}\right) e_{\perp}^{\mu} \Sigma_{A 2}^{-\rho \rho}\right) \\
& \left.\left.+\frac{\not P \perp}{P^{2} N^{2}}\left(-N^{2} P^{\mu} \Sigma_{A 3}^{-\rho \rho}+P^{2} N^{\mu} \Sigma_{A 2}^{-\rho \rho}+\rho \epsilon\left(p_{0}\right) e_{\perp}^{\mu} \Sigma_{A 1}^{-\rho \rho}\right)\right\}\right]
\end{aligned}
$$




$$
\begin{aligned}
& \mathcal{P}_{\rho} \gamma_{5} \frac{\partial X}{\partial P_{\alpha}} \frac{\partial C_{\rho-\rho} \Sigma_{A}^{-\rho-\rho}}{\partial X^{\alpha}} \mathcal{P}_{-\rho}=\mathcal{P}_{\rho} \frac{\partial N_{\mu}}{\partial P_{\alpha}} \gamma_{5} \frac{\partial}{\partial X^{\alpha}}\left[C _ { \rho - \rho } \left\{P^{\mu} \Sigma_{A 2}^{-\rho-\rho}+N^{\mu} \Sigma_{A 3}^{-\rho-\rho}-\rho \epsilon\left(p_{0}\right) e_{\perp}^{\mu} \Sigma_{A 4}^{-\rho-\rho}\right.\right. \\
& +\frac{\not P}{P^{2}}\left(P^{\mu} \Sigma_{A 1}^{-\rho-\rho}-P^{2} N^{\mu} \Sigma_{A 4}^{-\rho-\rho}+\rho \epsilon\left(p_{0}\right) e_{\perp}^{\mu} \Sigma_{A 3}^{-\rho-\rho}\right) \\
& +\frac{\not \supset}{N^{2}}\left(N^{2} P^{\mu} \Sigma_{A 4}^{-\rho-\rho}+N^{\mu} \Sigma_{A 1}^{-\rho-\rho}-\rho \epsilon\left(p_{0}\right) e_{\perp}^{\mu} \Sigma_{A 2}^{-\rho-\rho}\right) \\
& \left.\left.+\frac{\not P X}{P^{2} N^{2}}\left(N^{2} P^{\mu} \Sigma_{A 3}^{-\rho-\rho}-P^{2} N^{\mu} \Sigma_{A 2}^{-\rho-\rho}+\rho \epsilon\left(p_{0}\right) e_{\perp}^{\mu} \Sigma_{A 1}^{-\rho-\rho}\right)\right\}\right] \text {, } \\
& \mathcal{P}_{\rho} \frac{\partial \Sigma_{R}^{\rho \rho} C_{\rho-\rho}}{\partial X^{\alpha}} \frac{\partial X}{\partial P_{\alpha}} \gamma_{5} \mathcal{P}_{-\rho}=-\mathcal{P}_{\rho} \frac{\partial N_{\mu}}{\partial P_{\alpha}} \gamma_{5} \frac{\partial}{\partial X^{\alpha}}\left[\left\{-P^{\mu} \Sigma_{R 2}^{\rho \rho}-N^{\mu} \Sigma_{R 3}^{\rho \rho}-\rho \epsilon\left(p_{0}\right) e_{\perp}^{\mu} \Sigma_{R 4}^{\rho \rho}\right.\right. \\
& +\frac{\not P}{P^{2}}\left(P^{\mu} \Sigma_{R 1}^{\rho \rho}+\rho \epsilon\left(p_{0}\right) e_{\perp}^{\mu} \Sigma_{R 3}^{\rho \rho}+P^{2} N^{\mu} \Sigma_{R 4}^{\rho \rho}\right) \\
& +\frac{\perp^{\top}}{N^{2}}\left(N^{\mu} \Sigma_{R 1}^{\rho \rho}-\rho \epsilon\left(p_{0}\right) e_{\perp}^{\mu} \Sigma_{R 2}^{\rho \rho}-N^{2} P^{\mu} \Sigma_{R 4}^{\rho \rho}\right) \\
& \left.\left.+\frac{\not P X}{P^{2} N^{2}}\left(\rho \epsilon\left(p_{0}\right) e_{\perp}^{\mu} \Sigma_{R 1}^{\rho \rho}-P^{2} N^{\mu} \Sigma_{R 2}^{\rho \rho}+N^{2} P^{\mu} \Sigma_{R 3}^{\rho \rho}\right)\right\} C_{\rho-\rho}\right] \text {, } \\
& \mathcal{P}_{\rho} \frac{\partial \Sigma_{R}^{\rho-\rho} C_{-\rho \rho}}{\partial X^{\alpha}} \frac{\partial X}{\partial P_{\alpha}} \gamma_{5} \mathcal{P}_{\rho}=-\mathcal{P}_{\rho} \frac{\partial N_{\mu}}{\partial P_{\alpha}} \frac{\partial}{\partial X^{\alpha}}\left[\left\{-P^{\mu} \Sigma_{R 2}^{\rho-\rho}-N^{\mu} \Sigma_{R 3}^{\rho-\rho}+\rho \epsilon\left(p_{0}\right) e_{\perp}^{\mu} \Sigma_{R 4}^{\rho-\rho}\right.\right. \\
& +\frac{\not P}{P^{2}}\left(P^{\mu} \Sigma_{R 1}^{\rho-\rho}-\rho \epsilon\left(p_{0}\right) e_{\perp}^{\mu} \Sigma_{R 3}^{\rho-\rho}+P^{2} N^{\mu} \Sigma_{R 4}^{\rho-\rho}\right) \\
& +\frac{\not \mathcal{X}}{N^{2}}\left(N^{\mu} \Sigma_{R 1}^{\rho-\rho}+\rho \epsilon\left(p_{0}\right) e_{\perp}^{\mu} \Sigma_{R 2}^{\rho-\rho}-N^{2} P^{\mu} \Sigma_{R 4}^{\rho-\rho}\right) \\
& \left.\left.+\frac{\not P D}{P^{2} N^{2}}\left(-\rho \epsilon\left(p_{0}\right) e_{\perp}^{\mu} \Sigma_{R 1}^{\rho-\rho}-P^{2} N^{\mu} \Sigma_{R 2}^{\rho-\rho}+N^{2} P^{\mu} \Sigma_{R 3}^{\rho-\rho}\right)\right\} C_{-\rho \rho}\right], \\
& \mathcal{P}_{\rho} \gamma_{5} \mathcal{X}\left\{C_{\rho-\rho}, \Sigma_{A}^{-\rho \rho}\right\} \mathcal{P}_{\rho}=-\mathcal{P}_{\rho}\left[N^{2}\left\{C_{\rho-\rho}, \Sigma_{A 3}^{-\rho \rho}\right\}-N^{2} \not P\left\{C_{\rho-\rho}, \Sigma_{A 4}^{-\rho \rho}\right\}\right. \\
& \left.+\not \mathcal{X}\left\{C_{\rho-\rho}, \Sigma_{A 1}^{-\rho \rho}\right\}-\not P X\left\{C_{\rho-\rho}, \Sigma_{A 2}^{-\rho \rho}\right\}\right] \mathcal{P}_{\rho} \\
& -\frac{\partial C_{\rho-\rho}}{\partial X^{\alpha}} \mathcal{P}_{\rho}\left[N^{\alpha} \Sigma_{A 2}^{-\rho \rho}+\frac{1}{2} \frac{\partial N^{2}}{\partial P_{\alpha}} \Sigma_{A 3}^{-\rho \rho}-\rho \epsilon\left(p_{0}\right) e_{\perp}^{\mu} \frac{\partial N_{\mu}}{\partial P_{\alpha}} \Sigma_{A 4}^{-\rho \rho}\right. \\
& +\frac{\not P}{P^{2}}\left(\rho \epsilon\left(p_{0}\right) e_{\perp}^{\alpha} \Sigma_{A 2}^{-\rho \rho}+\rho \epsilon\left(p_{0}\right) e_{\perp}^{\mu} \frac{\partial N_{\mu}}{\partial P_{\alpha}} \Sigma_{A 3}^{-\rho \rho}-N^{2} \hat{P}^{\alpha} \Sigma_{A 4}^{-\rho \rho}\right) \\
& \left.+\frac{\not P D}{P^{2}}\left(-P^{\alpha} \Sigma_{A 2}^{-\rho \rho}+N^{\alpha} \Sigma_{A 3}^{-\rho \rho}+\rho \epsilon\left(p_{0}\right) e_{\perp}^{\alpha} \Sigma_{A 4}^{-\rho \rho}\right)\right] \mathcal{P}_{\rho}, \\
& \mathcal{P}_{\rho} \gamma_{5} \mathcal{X}^{T}\left\{C_{\rho-\rho}, \Sigma_{A}^{-\rho-\rho}\right\} \mathcal{P}_{-\rho}=\mathcal{P}_{\rho} \gamma_{5}\left[N^{2}\left\{C_{\rho-\rho}, \Sigma_{A 3}^{-\rho-\rho}\right\}-N^{2} \not P\left\{C_{\rho-\rho}, \Sigma_{A 4}^{-\rho-\rho}\right\}\right. \\
& +\mathbb{N}\left\{C_{\rho-\rho}, \Sigma_{A 1}^{-\rho-\rho}\right\}-\not P D\left\{\left\{C_{\rho-\rho}, \Sigma_{A 2}^{-\rho-\rho}\right\}\right] \mathcal{P}_{-\rho}
\end{aligned}
$$




$$
\begin{aligned}
& +\frac{\partial C_{\rho-\rho}}{\partial X^{\alpha}} \mathcal{P}_{\rho} \gamma_{5}\left[N^{\alpha} \Sigma_{A 2}^{-\rho-\rho}+\frac{1}{2} \frac{\partial N^{2}}{\partial P_{\alpha}} \Sigma_{A 3}^{-\rho-\rho}+\rho \epsilon\left(p_{0}\right) e_{\perp}^{\mu} \frac{\partial N_{\mu}}{\partial P_{\alpha}} \Sigma_{A 4}^{-\rho-\rho}\right. \\
& +\frac{\not P}{P^{2}}\left(-\rho \epsilon\left(p_{0}\right) e_{\perp}^{\alpha} \Sigma_{A 2}^{-\rho-\rho}-\rho \epsilon\left(p_{0}\right) e_{\perp}^{\mu} \frac{\partial N_{\mu}}{\partial P_{\alpha}} \Sigma_{A 3}^{-\rho-\rho}-N^{2} \hat{P}^{\alpha} \Sigma_{A 4}^{-\rho-\rho}\right) \\
& \left.+\frac{\not P X}{P^{2}}\left(-P^{\alpha} \Sigma_{A 2}^{-\rho-\rho}+N^{\alpha} \Sigma_{A 3}^{-\rho-\rho}-\rho \epsilon\left(p_{0}\right) e_{\perp}^{\alpha} \Sigma_{A 4}^{-\rho-\rho}\right)\right] \mathcal{P}_{-\rho}, \\
& \mathcal{P}_{\rho}\left\{C_{\rho-\rho}, \Sigma_{R}^{\rho \rho}\right\} \not \mathcal{N} \gamma_{5} \mathcal{P}_{-\rho}=\mathcal{P}_{\rho} \gamma_{5}\left[N^{2}\left\{C_{\rho-\rho}, \quad \Sigma_{R 3}^{\rho \rho}\right\}-N^{2} \not P\left\{C_{\rho-\rho}, \quad \Sigma_{R 4}^{\rho \rho}\right\}\right. \\
& \left.-\mathbb{X}\left\{C_{\rho-\rho}, \Sigma_{R 1}^{\rho \rho}\right\}+\not P D T\left\{C_{\rho-\rho}, \Sigma_{R 2}^{\rho \rho}\right\}\right] \mathcal{P}_{-\rho} \\
& +\frac{\partial C_{\rho-\rho}}{\partial X^{\alpha}} \mathcal{P}_{\rho} \gamma_{5}\left[N^{\alpha} \Sigma_{R 2}^{\rho \rho}+\frac{1}{2} \frac{\partial N^{2}}{\partial P_{\alpha}} \Sigma_{R 3}^{\rho \rho}-\rho \epsilon\left(p_{0}\right) e_{\perp}^{\mu} \frac{\partial N_{\mu}}{\partial P_{\alpha}} \Sigma_{R 4}^{\rho \rho}\right. \\
& +\frac{\not P}{P^{2}}\left(\rho \epsilon\left(p_{0}\right) e_{\perp}^{\alpha} \Sigma_{R 2}^{\rho \rho}+\rho \epsilon\left(p_{0}\right) e_{\perp}^{\mu} \frac{\partial N_{\mu}}{\partial P_{\alpha}} \Sigma_{R 3}^{\rho \rho}-N^{2} \hat{P}^{\alpha} \Sigma_{R 4}^{\rho \rho}\right) \\
& \left.+\frac{\not P D}{P^{2}}\left(P^{\alpha} \Sigma_{R 2}^{\rho \rho}-N^{\alpha} \Sigma_{R 3}^{\rho \rho}-\rho \epsilon\left(p_{0}\right) e_{\perp}^{\alpha} \Sigma_{R 4}^{\rho \rho}\right)\right] \mathcal{P}_{-\rho}, \\
& \mathcal{P}_{\rho}\left\{C_{-\rho \rho}, \Sigma_{R}^{\rho-\rho}\right\} \mathbb{N} \gamma_{5} \mathcal{P}_{\rho}=\mathcal{P}_{\rho}\left[N^{2}\left\{C_{-\rho \rho}, \quad \Sigma_{R 3}^{\rho-\rho}\right\}-N^{2} \not P\left\{C_{\rho-\rho}, \quad \Sigma_{R 4}^{\rho-\rho}\right\}\right. \\
& \left.-X \mathcal{X}\left\{C_{\rho-\rho}, \quad \Sigma_{R 1}^{\rho-\rho}\right\}+\not P X\left\{C_{\rho-\rho}, \Sigma_{R 2}^{\rho-\rho}\right\}\right] \mathcal{P}_{\rho} \\
& +\frac{\partial C_{-\rho \rho}}{\partial X^{\alpha}} \mathcal{P}_{\rho}\left[N^{\alpha} \Sigma_{R 2}^{\rho-\rho}+\frac{1}{2} \frac{\partial N^{2}}{\partial P_{\alpha}} \Sigma_{R 3}^{\rho-\rho}+\rho \epsilon\left(p_{0}\right) e_{\perp}^{\mu} \frac{\partial N_{\mu}}{\partial P_{\alpha}} \Sigma_{R 4}^{\rho-\rho}\right. \\
& +\frac{\not P}{P^{2}}\left(-\rho \epsilon\left(p_{0}\right) e_{\perp}^{\alpha} \Sigma_{R 2}^{\rho-\rho}-\rho \epsilon\left(p_{0}\right) e_{\perp}^{\mu} \frac{\partial N_{\mu}}{\partial P_{\alpha}} \Sigma_{R 3}^{\rho-\rho}-N^{2} \hat{P}^{\alpha} \Sigma_{R 4}^{\rho-\rho}\right) \\
& \left.+\frac{\not P D}{P^{2}}\left(P^{\alpha} \Sigma_{R 2}^{\rho-\rho}-N^{\alpha} \Sigma_{R 3}^{\rho-\rho}+\rho \epsilon\left(p_{0}\right) e_{\perp}^{\alpha} \Sigma_{R 4}^{\rho-\rho}\right)\right] \mathcal{P}_{\rho} .
\end{aligned}
$$

Standard forms for $\gamma_{5} \underline{\underline{X}} \cdot \underline{\mathbf{C}}$ and $\underline{\mathbf{C}} \cdot \gamma_{5} \underline{\not \perp}$ in $G_{K}^{[3]}$ in Eq. (4.20)

Form for $\gamma_{5} \underline{\underline{X}} \cdot \underline{\mathbf{C}}$ in $\mathbf{G}_{K}^{[3]}$ in Eq. (4.20) is given by Eq. (E.1) with

$$
\begin{aligned}
\Sigma_{A 1}^{\rho \sigma} \rightarrow \delta^{\rho \sigma}, \quad & \Sigma_{A j}^{\rho \sigma} \rightarrow 0 \quad(j=2-4), \\
\Sigma_{R j}^{\rho \sigma} \rightarrow 0 & (j=1-4) .
\end{aligned}
$$

$\underline{\mathrm{C}} \cdot \gamma_{5} \underline{\underline{X}}$ in Eq. (4.20) is given by Eq. (E.1) with

$$
\begin{aligned}
\Sigma_{R 1}^{\rho \sigma} \rightarrow-\delta^{\rho \sigma}, \quad & \sum_{R j}^{\rho \sigma} \rightarrow 0 \quad(j=2-4), \\
\Sigma_{A j}^{\rho \sigma} \rightarrow 0 & (j=1-4) .
\end{aligned}
$$




\section{APPENDIX F: ENERGY SHELLS OF $G_{R}^{\rho \rho}(P, X)$}

To find the energy shells of $G_{R}^{\rho \rho}$, we need $\left(G_{R}^{\rho \rho}(P, X)\right)^{-1}$, the inverse of $G_{R}^{\rho \rho}(P, X)(\mathrm{cf}$. Eq. 4.28)). To the gradient approximation, we have

$$
\begin{aligned}
\left(G_{R}^{\rho \rho}\right)^{-1} & =\left(G_{R}^{(0) \rho \rho}+G_{R}^{(1) \rho \rho}\right)^{-1} \\
& \simeq\left(G_{R}^{(0) \rho \rho}\right)^{-1}-\left(G_{R}^{(0) \rho \rho}\right)^{-1} G_{R}^{(1) \rho \rho}\left(G_{R}^{(0) \rho \rho}\right)^{-1}
\end{aligned}
$$

Here $\left(G_{R}^{(0) \rho \rho}\right)^{-1}$ is the (11)-element of Eq. (2.48) and $G_{R}^{(1) \rho \rho}$ is as in Eq. (4.24). If we ignore the gradient term in Eq. (F.1), the energy shells are obtained through

$$
\left.\left.\operatorname{Re}\left(G_{R}^{(0) \rho \rho}(P, X)\right)^{-1}\right|_{p_{0}= \pm \omega_{ \pm}^{(0)}( \pm \vec{p}, X)} \propto \mathcal{D}^{\rho \rho}\right|_{p_{0}= \pm \omega_{ \pm}^{(0)}( \pm \vec{p}, X)}=0
$$

where $\mathcal{D}^{\rho \rho}$ is given by Eq. (2.46) with the substitutions (2.50) being made. Then, the true energy shells, $p_{0}= \pm \omega_{ \pm}( \pm \vec{p}, X)$, are obtained from Eq. (F.1),

$$
\begin{aligned}
& \pm\left.\frac{\partial \operatorname{Re}\left(G_{R}^{(0) \rho \rho}(P, X)\right)^{-1}}{\partial p_{0}}\right|_{p_{0}= \pm \omega_{ \pm}^{(0)}( \pm \vec{p}, X)}\left(\omega_{ \pm}( \pm \vec{p}, X)-\omega_{ \pm}^{(0)}( \pm \vec{p}, X)\right) \\
& \quad=\left.\operatorname{Re}\left[\left(G_{R}^{(0) \rho \rho}(P, X)\right)^{-1} G_{R}^{(1) \rho \rho}(P, X)\left(G_{R}^{(0) \rho \rho}(P, X)\right)^{-1}\right]\right|_{p_{0}= \pm \omega_{ \pm}^{(0)}( \pm \vec{p}, X)}
\end{aligned}
$$

\section{APPENDIX G: STANDARD FORMS FOR THE QUANTITIES IN SEC. IVB}

$$
\text { Standard forms for } \Pi_{K}^{[1] \mu \nu} \text { and } \Pi_{K}^{[2] \mu \nu} \text { in Eq. (4.64) }
$$

From Eq. (4.57) with Eq. (4.64), we obtain

$$
\begin{aligned}
& \Pi_{K}^{[1] \mu \nu}=\frac{i}{2} \sum_{j} \sum_{U V=T, L}\left[\mathcal{R}_{L j}^{U V}\left\{\tilde{f}, \tilde{\Pi}_{R j}^{U V}+\tilde{\Pi}_{A j}^{U V}\right\} \mathcal{R}_{R j}^{U V}\right]^{\mu \nu}, \\
& \Pi_{K}^{[2] \mu \nu}=2 i \mathcal{P}_{T}^{\mu \nu} \operatorname{Re}\left[\Pi_{R 1}^{T G}-\frac{\zeta \cdot \tilde{P}}{\tilde{P}^{2}} \Pi_{R 3}^{T^{\prime} T}\right]\left(E_{\perp} \cdot \partial\right) \tilde{f}+2 i \frac{\tilde{\zeta}^{\mu} \tilde{\zeta}^{\nu}}{\tilde{\zeta}^{2}} R e\left[\left(-\frac{\zeta \cdot \tilde{P}}{\tilde{P}^{2}} \Pi_{R 2}^{T T}+\Pi_{R 2}^{T G}\right)(\tilde{\zeta} \cdot \partial) \tilde{f}\right.
\end{aligned}
$$




$$
\begin{aligned}
& \left.+\left\{\frac{\zeta \cdot \tilde{P}}{\tilde{P}^{2}}\left(\Pi_{R 3}^{T^{\prime} T}-\Pi_{R 3}^{T T^{\prime}}\right)-\Pi_{R 1}^{T G}\right\}\left(E_{\perp} \cdot \partial\right) \tilde{f}\right] \\
& +i \tilde{\zeta}^{\mu} E_{\perp}^{\nu}\left[-\frac{1}{\tilde{P}^{2}} \Pi_{R 3}^{T T^{\prime}}(\tilde{P} \cdot \tilde{\partial}) \tilde{f}+\frac{1}{E_{\perp}^{2}}\left(\Pi_{R 2}^{T G}-\frac{\zeta \cdot \tilde{P}}{\tilde{P}^{2}} \Pi_{R 2}^{T T}\right)\left(E_{\perp} \cdot \partial\right) \tilde{f}\right. \\
& \left.+\frac{1}{\tilde{\zeta}^{2}}\left(\Pi_{A 1}^{G T}+\frac{\zeta \cdot \tilde{P}}{\tilde{P}^{2}}\left(\Pi_{R 3}^{T T^{\prime}}+\Pi_{A 3}^{T T^{\prime}}\right)\right)(\tilde{\zeta} \cdot \partial) \tilde{f}\right]+2 i n^{\mu} n^{\nu} R e \Pi_{R 1}^{L G} \partial_{0} \tilde{f} \\
& +i \tilde{\zeta}^{\mu} n^{\nu}\left[\Pi_{R 2}^{T G} \partial_{0} \tilde{f}-\frac{1}{\tilde{\zeta}^{2}}\left(\Pi_{A 1}^{G L}+\frac{\zeta \cdot \tilde{P}}{\tilde{P}^{2}} \Pi_{A 1}^{T L}\right)(\tilde{\zeta} \cdot \partial) \tilde{f}+\frac{\zeta \cdot \tilde{P}}{\tilde{P}^{2} \tilde{\zeta}^{2}} \Pi_{A 2}^{T L}\left(E_{\perp} \cdot \partial\right) \tilde{f}\right] \\
& +i E_{\perp}^{\mu} n^{\nu}\left[\frac{1}{\tilde{P}^{2}} \Pi_{A 2}^{T L}(\tilde{P} \cdot \tilde{\partial}) \tilde{f}+\Pi_{R 1}^{T G} \partial_{0} \tilde{f}-\frac{\zeta \cdot \tilde{P}}{\tilde{P}^{2} \tilde{\zeta}^{2}} \Pi_{A 2}^{T L}(\tilde{\zeta} \cdot \partial) \tilde{f}\right. \\
& \left.-\frac{1}{E_{\perp}^{2}}\left(\Pi_{A 1}^{G L}+\frac{\zeta \cdot \tilde{P}}{\tilde{P}^{2}} \Pi_{A 1}^{T L}\right)\left(E_{\perp} \cdot \partial\right) \tilde{f}\right] \\
& +i E_{\perp}^{\mu} \tilde{\zeta}^{\nu}[\ldots]+i n^{\mu} \tilde{\zeta}^{\nu}[\ldots]+i n^{\mu} E_{\perp}^{\nu}[\ldots] .
\end{aligned}
$$

[...]'s are obtained using Eq. (3.26).

Standard dorm for $\tilde{H}$ in Eq. (4.60)

We write $\underline{\tilde{H}}^{\mu \nu}=\underline{\tilde{H}}^{(0) \mu \nu}+\underline{\tilde{H}}^{(1) \mu \nu}$, with $\underline{\tilde{H}}^{(0) \mu \nu}$ the leading term and $\underline{\tilde{H}}^{(1) \mu \nu}$ the gradient term of the DEX of $\tilde{H}^{\mu \nu}(P, X)$. Straightforward manipulation of Eq. (4.60) yields

$$
\begin{aligned}
\underline{\tilde{H}}^{(0) \mu \nu}= & \underline{\mathcal{P}}_{T}^{\mu \rho} \cdot\left[\left(\tilde{H}_{l}\right)_{1}^{T T}\right]_{\mathrm{IWT}} \cdot\left(\underline{\mathcal{P}}_{T}\right)_{\rho}^{\nu}+\underline{\tilde{\zeta}}^{\mu} \cdot\left[\left(\tilde{H}_{l}\right)_{2}^{T T}\right]_{\mathrm{IWT}} \cdot \underline{\tilde{\zeta}}^{\nu}-\underline{\tilde{\zeta}}^{\mu} \cdot\left[\left(\tilde{H}_{l}\right)_{3}^{T T^{\prime}}\right]_{\mathrm{IWT}} \cdot \underline{E}_{\perp}^{\nu} \\
& +\underline{E}_{\perp}^{\mu} \cdot\left[\left(\tilde{H}_{l}\right)_{3}^{T^{\prime} T}\right]_{\mathrm{IWT}} \cdot \tilde{\tilde{\zeta}}^{\nu}+\underline{\tilde{\zeta}}^{\mu} \cdot\left[\left(\tilde{H}_{l}\right)_{1}^{T L}\right]_{\mathrm{IWT}} n^{\nu}+\underline{E}_{\perp}^{\mu} \cdot\left[\left(\tilde{H}_{l}\right)_{2}^{T L}\right]_{\mathrm{IWT}} n^{\nu} \\
& +n^{\mu}\left[\left(\tilde{H}_{l}\right)_{1}^{L T}\right]_{\mathrm{IWT}} \cdot \underline{\tilde{\zeta}}^{\nu}-n^{\mu}\left[\left(\tilde{H}_{l}\right)_{2}^{L T}\right]_{\mathrm{IWT}} \cdot \underline{E}_{\perp}^{\nu}
\end{aligned}
$$

with $\left(\tilde{H}_{l}\right)_{j}^{U V}$ as in Eq. (3.55), and

$$
\begin{aligned}
\tilde{H}^{(1) \mu \nu}= & i \mathcal{P}_{T}^{\mu \nu} \operatorname{Re}\left[\tilde{\zeta}^{2} E_{\perp}^{2}\left\{C_{3}^{T T^{\prime}}, \Pi_{R 3}^{T^{\prime} T}\right\}\right. \\
& \left.-2(\zeta \cdot \tilde{P})\left\{\tilde{\zeta}^{2} C_{3}^{T T^{\prime}}(\tilde{\zeta} \cdot \stackrel{\leftrightarrow}{\partial}) \Pi_{R 3}^{T^{\prime} T}-\frac{1}{\tilde{P}^{2}} C_{3}^{T T^{\prime}}\left(E_{\perp} \cdot \partial\right) \Pi_{R 1}^{T T}\right\}\right] \\
& +i \tilde{\zeta}^{\mu} \tilde{\zeta}^{\nu} \operatorname{Re}\left[-\left\{C_{2}^{T T}, \Pi_{R 1}^{T T}\right\}-\tilde{\zeta}^{2}\left\{C_{2}^{T T}, \Pi_{R 2}^{T T}\right\}+E_{\perp}^{2}\left\{C_{3}^{T^{\prime} T}, \Pi_{R 3}^{T T^{\prime}}-\Pi_{A 3}^{T T^{\prime}}\right\}\right. \\
& -2 \frac{\zeta \cdot \tilde{P}}{\tilde{P}^{2}} C_{2}^{T T}(\tilde{\zeta} \cdot \stackrel{\leftrightarrow}{\partial}) \Pi_{R 2}^{T T}+2 \tilde{\zeta}^{2} C_{3}^{T^{\prime} T}(\tilde{P} \cdot \stackrel{\leftrightarrow}{\partial}) \Pi_{R 3}^{T T^{\prime}} \\
& +2(\zeta \cdot \tilde{P})\left(C_{3}^{T T^{\prime}}(\tilde{\zeta} \cdot \stackrel{\leftrightarrow}{\partial}) \Pi_{R 3}^{T^{\prime} T}-C_{3}^{T^{\prime} T}(\tilde{\zeta} \cdot \stackrel{\leftrightarrow}{\partial}) \Pi_{R 3}^{T T^{\prime}}\right)
\end{aligned}
$$




$$
\begin{aligned}
& \left.-2 \frac{\zeta \cdot \tilde{P}}{\tilde{P}^{2} \tilde{\zeta}^{2}}\left(C_{2}^{T T}(\tilde{\zeta} \cdot \partial)+2 R e C_{3}^{T T^{\prime}}\left(E_{\perp} \cdot \partial\right)\right) \Pi_{R 1}^{T T}\right] \\
& +\frac{i}{2} \frac{\tilde{\zeta}^{\mu} E_{\perp}^{\nu}}{\tilde{P}^{2}}\left[\tilde{P}^{2} \tilde{\zeta}^{2}\left(\left\{C_{2}^{T T}, \Pi_{A 3}^{T T^{\prime}}\right\}+\left\{C_{3}^{T T^{\prime}}, \Pi_{R 2}^{T T}\right\}\right)+2 \tilde{P}^{2}\left\{C_{3}^{T T^{\prime}}, \operatorname{Re} \Pi_{R 1}^{T T}\right\}\right. \\
& -2 C_{3}^{T T^{\prime}}(\tilde{P} \cdot \tilde{\partial}) \Pi_{A 1}^{T T}+2(\zeta \cdot \tilde{P})\left\{-C_{2}^{T T} \frac{E_{\perp} \cdot \partial}{E_{\perp}^{2}} \Pi_{A 1}^{T T}+C_{2}^{T T}(\tilde{\zeta} \cdot \stackrel{\leftrightarrow}{\partial}) \Pi_{A 3}^{T T^{\prime}}\right. \\
& \left.\left.+C_{3}^{T T^{\prime}}(\tilde{\zeta} \cdot \stackrel{\leftrightarrow}{\partial}) \Pi_{R 2}^{T T}+2 i\left(E_{\perp} \cdot \partial\right) I m\left(C_{3}^{T T^{\prime}} \Pi_{R 3}^{T^{\prime} T}\right)+\frac{2}{\tilde{\zeta}^{2}} C_{3}^{T T^{\prime}}(\tilde{\zeta} \cdot \partial) R e \Pi_{R 1}^{T T}\right\}\right] \\
& +\frac{i}{2} \tilde{\zeta}^{\mu} n^{\nu}\left[-\tilde{\zeta}^{2}\left\{C_{2}^{T T}, \Pi_{A 1}^{T L}\right\}+E_{\perp}^{2}\left\{C_{3}^{T T^{\prime}}, \Pi_{A 2}^{T L}\right\}\right. \\
& \left.-\frac{2 \zeta \cdot \tilde{P}}{\tilde{P}^{2}} C_{2}^{T T}(\tilde{\zeta} \cdot \stackrel{\leftrightarrow}{\partial}) \Pi_{A 1}^{T L}+2 C_{3}^{T T^{\prime}}\left(\tilde{\zeta}^{2}(\tilde{P} \cdot \stackrel{\leftrightarrow}{\partial})-(\zeta \cdot \tilde{P})(\tilde{\zeta} \cdot \stackrel{\leftrightarrow}{\partial})\right) \Pi_{A 2}^{T L}\right] \\
& -i E_{\perp}^{\mu} n^{\nu}\left[\frac{\tilde{\zeta}^{2}}{2}\left\{C_{3}^{T^{\prime} T}, \Pi_{A 1}^{T L}\right\}+\frac{\tilde{\zeta}^{2} \cdot \tilde{P}}{\tilde{P}^{2}} C_{3}^{T^{\prime} T}(\tilde{\zeta} \cdot \stackrel{\leftrightarrow}{\partial}) \Pi_{A 1}^{T L}\right] \\
& +i E_{\perp}^{\mu} \tilde{\zeta}^{\nu}[\ldots]+i n^{\mu} \tilde{\zeta}^{\nu}[\ldots]-i n^{\mu} E_{\perp}^{\nu}[\ldots],
\end{aligned}
$$

where $A \stackrel{\leftrightarrow}{\partial} B \equiv A \partial B-A \overleftarrow{\partial} B$, and [...]'s are obtained using Eqs. (3.32) and (3.35). 


\section{REFERENCES}

[1] J. J. Drake et al., Astrophys. J. 572, 996 (2002).

[2] See also, K. Kohri, K. Iida, and K. Sato, Prog. Theor. Phys. 109, 765 (2003), and references therein.

[3] T. Tatsumi, Phys. Lett. B489, 280 (2000); astroph/0004062.

[4] J. Schwinger, J. Math. Phys. 2, 407 (1961); L. V. Keldysh, Zh. Eksp. Teor. Fiz. 47, 1515 (1964) (Sov. Phys. JETP 20, 1018 (1965)); R. A. Craig, J. Math. Phys. 9, 605 (1968); J. Rammer and H. Smith, Rev. Mod. Phys. 58, 323 (1986).

[5] K.-C. Chou, Z.-B. Su, B.-L. Hao, and L. Yu, Phys. Rep. 118, 1 (1985).

[6] H. Umezawa, "Advanced Field Theory - Micro, Macro, and Thermal Physics." AIP, New York, 1993; H. Chu and H. Umezawa, Int. J. Mod. Phys. A9, 1703 and 2363 (1994) and earlier papers quoted therein.

[7] K. Okano, Mod. Phys. Lett. A16, 531 (2001). See also, M. Dirks, A. Niégawa, and K. Okano, Phys. Lett. B461, 131 (1999).

[8] A. Niegawa, Prog. Theor. Phys. 102, 1 (1999).

[9] A. Niegawa, Phys. Rev. D 65, 056009 (2002).

[10] A. Niegawa, Phys. Rev. D 64, 036004 (2001).

[11] T. Arimitsu and H. Umezawa, Prog. Theor. Phys. 77, 32 (1987).

[12] T. Altherr and D. Seibert, Phys. Lett. B333, 149 (1994); T. Altherr, ibid. B341, 325 (1995).

[13] P. M. Stevenson, Phys. Rev. D 23, 2916 (1981). 